\title{
Low-Frequency Bottom Backscattering Data Analysis Using Multiple Constraints Beamforming
} by

\author{
Dan Li \\ B. S., Electrical Fngineering \\ University of Science and Technology of China (1992) \\ Submitted in partial fulfillment of the \\ requirements for the dual degrees of \\ MASTER OF SCIENCE IN OCEANOGRAPHIC ENGINEERING \\ and \\ OCEAN ENGINEER IN OCEANOGRAPHIC ENGINEERING \\ at the \\ MASSACHUSETTS INSTITUTE OF TECHNOLOGY \\ and the \\ WOODS HOLE OCEANOGRAPHIC INSTITUTION \\ May 1995 \\ (C) Dan Li, 1995. All rights reserved.
}

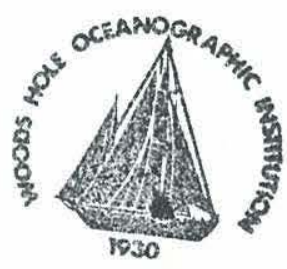

The author hereby grants to MIT and WHOI permission to reproduce and to distribute copies of this thesis document in whole or in part.

Signature of Author

Department of Ocean Engineering, MIT and the MIT/WHOI Joint Programpin Oceanographic Engineering

Certified by

Certified by

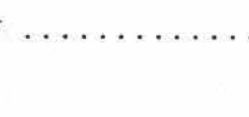

Assistant Scientist, Woods Hole Oceanographic Institution U 'Dr. Dajún Tang Thesis Supervisor ?..... $\cup$ Dr. George V. Frisk Senior Scientist, Woods Hole Oceanographic Institution Thesis Supervisor

Certified by

Professor of Ocean Engineering, Massachusetts Instifute of Technology
Thesis Reader Accepted by

Professor Arthur B. Baggeroer Chairman, Joint Committee for Oceanographic Engineering, Massachusetts Institute of Technology-Woods Hole Oceanographic Institution 


\title{
Low-Frequency Bottom Backscattering Data Analysis Using Multiple Constraints Beamforming \\ by Dan Li
}

Submitted to the Massachusetts Institute of Technology/

Woods Hole Oceanographic Institution

Joint Program in Oceanographic Engineering

in May, 1995 in partial fulfillment of the

requirements for the dual degrees of

\author{
MASTER OF SCIENCE IN OCEANOGRAPHIC ENGINEERING \\ and
}

OCEAN ENGINEER IN OCEANOGRAPHIC ENGINEERING

\begin{abstract}
The data analysis of a deep-sea bottom backscattering experiment, carried out over a sediment pond on the western flank of the Mid-Atlantic Ridge in July 1993 with a 250$650 \mathrm{~Hz}$ chirp source and a vertical receiving array suspended near the flat seafloor, is presented in this thesis. Reflected signals in the normal incidence direction as the output of endfire beamforming are used to determine the sediment structure. The sediment is found to be horizontally stratified, except for two irregular regions, each about $20 \mathrm{~m}$ thick, located around $18 \mathrm{~m}$ and $60 \mathrm{~m}$ beneath the water-sediment interface. Multiple constraints beamforming is shown to be effective in removing coherent reflections from internal stratified layers, which is critical to the analysis of bottom backscattering. With backscattered signals obtained by beamforming, the above-mentioned two inhomogeneous regions are found to be the dominant factors on the bottom backscattered field, both in the normal incidence and oblique directions. The backscattering strength as a function of grazing angle is estimated for each of the two regions.
\end{abstract}

Thesis Supervisor: Dr. Dajun Tang

Assistant Scientist

Woods Hole Oceanographic Institution

Thesis Supervisor: Dr. George V. Frisk

Senior Scientist

Woods Hole Oceanographic Institution 


\section{Acknowledgments}

I am very grateful to my thesis advisors Dr. Dajun Tang and Dr. George V. Frisk for guiding me into a new field - the world of sound. It is their patience, trust and encouragement that lead to all of those joyful moments during my thesis work. My indebtedness also goes to Prof. Henrik Schmidt, my thesis reader, for his insightful suggestions, to Dr. James C. Preisig, for his excellent advice on the application of array signal processing technique.

I would like to express my gratitude to my academic advisor at MIT, Prof. Arthur B. Baggeroer, and all the MIT/WHOI Joint Program staffs for their continuous support. I really appreciate the help of Cynthia J. Sellers from WHOI for managing our computer system and the ARSRP data.

Thanks also goes to Dr. William S. Hodgkiss of the Scripps Institution of Oceanography and Dr. Joseph F. Gettrust of the Naval Research Laboratory for their extensive contributions to the experiment, and to Dr. Subramaniam D. Rajan and Dr. William C. Burgess of WHOI for their involvement in the experiment and valuable discussions.

To my fellow students in 5-007, thanks for a lot of wonderful discussions and special thanks to Brian H. Tracey for sanity-checking my work and proofreading my thesis.

To my friends at MIT, life can never be the same without all the fun that we have together. Qing, Henry, Chenyang, Feng, Xiaowei, Wenjie, Lan ...it would be a long list and the shapeless names are just not enough to show my appreciation.

Finally, I wish to express my most grateful thanks to my parents, my sister Ying, for their invaluable spiritual support, and to my friends Helen Huang and Xiaoou Tang, for giving me the best that one can expect from friends. Without their understanding and encouragement, I would not have been able to complete this work successfully. 


\section{Contents}

1 Introduction $\quad 9$

1.1 General Introduction . . . . . . . . . . . . . . . . . . . 9

1.2 Relevant Literature Review . . . . . . . . . . . . . . . . . . . 12

1.2.1 Volume Backscattering Models . . . . . . . . . . . . . 12

1.2.2 Experimental Data Analysis . . . . . . . . . . . . 18

1.3 Outline of Thesis . . . . . . . . . . . . . . . . . 20

2 Experiment Description and Theory 22

2.1 Experiment Description . . . . . . . . . . . . . . . 22

2.2 Beamforming and Simulations . . . . . . . . . . . . . . 26

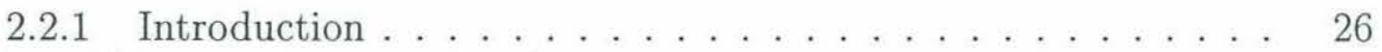

2.2.2 Single and Multiple Constraints Beamforming . . . . . . . . . 29

2.2.3 Simulation . . . . . . . . . . . . . . . . 35

2.3 Array Element Localization . . . . . . . . . . . . . . . . . . . . . 43

2.4 Bottom Backscattering Strength . . . . . . . . . . . . . . 48

3 Experimental Data Analysis Results 52

3.1 Preprocessing . . . . . . . . . . . . . . . . 52

3.2 Analysis of Signals in the Normal Incidence Direction . . . . . . . . . 55

3.3 Analysis of Signals in Oblique Directions . . . . . . . . . . . . 66

4 Conclusions and Future Work $\quad 79$

4.1 Conclusions . . . . . . . . . . . . . . . . . . . . . . . 79 
4.2 Recommendations for Future Work . . . . . . . . . . . . . . 


\section{List of Figures}

2-1 Bathymetry and ship track in the ARSRP Site A sediment pond. . . 24

2-2 The experimental scenario. . . . . . . . . . . . . . . . 25

2-3 The DTAGS source and receiving array geometry. . . . . . . . . . 26

2-4 (a) The received signal and (b) its spectrum at one hydrophone for a typical ping ........................... 27

2-5 The estimated receiving array configuration for a typical ping . . . . 28

2-6 The linear beamforming scheme. . . . . . . . . . . . . . . . 30

2-7 The geometry of a vertical line array. . . . . . . . . . . . . . . 31

2-8 The beampattern for a single-constraint beamformer: frequency $=450$ $\mathrm{Hz}$, look direction $=-45$ degrees. . . . . . . . . . . . 34

2-9 The beampattern for a five-constraint beamformer: frequency $=450$ $\mathrm{Hz}$, look direction $=-45$ degrees. . . . . . . . . . . 35

2-10 The geometry of scatterers. . . . . . . . . . . . . . 36

2-11 The simulated source signal: (a) the waveform; (b) the spectrum. . . 37

2-12 The simulated time series at the bcttom hydrophone. . . . . . . . . 38

2-13 (a) The returned signal from the target alone; (b) The signals from the scatterers in the second layer; (c) Part of (d) the entire received signals at the bottom hydrophone. . . . . . . . . . . . . . .

2-14 The single-constraint beamforming results: (a) the received signals at the bottom hydrophone (dashed line) and the beamformer output (solid line); (b) the target signal (dashed line) and the beamformer output (solid line). . . . . . . . . . . . . . . . . . 
2-15 The multiple-constraint beamforming results: (a) the received signals at the bottom hydrophone (dashed line) and the beamformer output (solid line); (b) the target signal (dashed line) and the beamformer output (solid line). . . . . . . . . . . . . . . . . . . 41

2-16 The hybrid method results: (a) the received signals at the bottom hydrophone (dashed line) and the hybrid method output (solid line); (b) the target signal (dashed line) and the hybrid method output (solid line)............................... 42

2-17 Scheme of the array element localization algorithm. . . . . . . . . . 44

2-18 Hydrophone positions: the horizontal range from the source and the height above the seafloor. . . . . . . . . . . . . . . 47

2-19 The conventional single source/single receiver configuration. . . . . . 49

3-1 (a) The bandpass filter output and (b) its spectrum for the signals at one hydrophone for a typical ping. . . . . . . . . . . . . . 53

3-2 The signals at 24 hydrophones after matched filtering for a typical ping 54

3-3 Beampattern for the endfire beamformer at $450 \mathrm{~Hz}$. . . . . . . . 57

3-4 Beampattern for the endfire beamformer at $250 \mathrm{~Hz} \ldots \ldots$. . . . 57

3-5 Beampattern for the endfire beamformer at $650 \mathrm{~Hz}$. . . . . . . . . 58

3-6 For a typical ping: (a) the signal on the bottom hydrophone after matched filtering, (b) the return in the normal direction obtained by endfire beamforming, (c) the difference signal between (a) and (b) . . 59

3-7 The cross-section of part of the sediment pond. . . . . . . . . . . . 61

3-8 (a) Beamformer outputs in the normal incidence direction for 8 pings at the east side of the sediment pond, (b) the mean of the signals in (a), (c) the signals in (a) with the mean removed. . . . . . . . . . .

3-9 (a) Beamformer outputs in the normal incidence direction for 8 pings at the west side of the sediment pond, (b) the mean of the signals in (a), (c) the signals in (a) with the mean removed. . . . . . . . . . . 
3-10 The inferred sediment structure using the edge detection algorithm for 9 groups of real data . . . . . . . . . . . . . . . . 65

3-11 For a typical ping: the time series at channel 1 (a) before and (b) after the subtraction. . . . . . . . . . . . . . . . . . 68

3-12 The envelope of oblique angle beamforming results for a single ping at the east side of the sediment pond (arbitrary units) . . . . . . . 70

3-13 The average envelope of oblique angle beamforming results over 8 pings at the east side of the sediment pond (arbitrary units). . . . . . . . 71

3-14 The envelope of oblique angle beamforming results for a single ping at the west side of the sediment pond (arbitrary units). . . . . . . . . 72

3-15 The average envelope of oblique angle beamforming results over 8 pings at the west side of the sediment pond (arbitrary units) . . . . . . . 73

3-16 The backscattering strength with standard deviations (error bars) and the Lambert's law fit (dashed line) for the (a) upper and (b) lower irregular regions at the east side of the sediment pond. . . . . . . . . 76

3-17 The backscattering strength with standard deviations (error bars) and the Lambert's law fit (dashed line) for the lower irregular region at the west side of the sediment pond. . . . . . . . . . . . . . 


\section{Chapter 1}

\section{Introduction}

\section{$1.1 \quad$ General Introduction}

During the past several decades, sound scattering has obtained more and more attention in underwater acoustics and is a very important part of ongoing research. Interests vary from applications such as target detection and underwater obstruction avoidance, to the study of fish groups and zooplankton, to geological surveys of the ocean bottom, to monitoring of environmental pollution. Many efforts have been put into understanding the physical mechanisms that cause scattering of acoustic waves within the ocean and from its boundaries, which remain unclear at the present stage. This is because the ocean is an extremely complicated acoustic system. The scattering of the propagating sound in it depends on many factors such as frequency, incident angle, the type of scatterers and even some unknown physical processes.

Usually, acoustic scattering can be divided into the following categories:

- Ocean surface scattering.

- Volumetric scattering in the water column.

- Ocean bottom scattering.

The emphasis of the research presented here will be put on ocean bottom scattering. 
The principal components of backscattering from the ocean bottom are generally considered to be:

1. Backscattering from the water-bottom interface due to surface roughness.

2. Backscattering from inhomogeneities within the bottom.

Ocean bottom interface roughness can be ascribed to large topographical features such as undersea mountains and ridges, and small features such as sand ripples. It is easy to understand that large topographical features are the dominant contributors to bottom backscattering in many regions. However, for sediment covered areas, where only small interface roughness features exist, the influence of bottom inhomogeneities on the total bottom backscattered field is less obvious and less well understood. Inhomogeneities within the sediment that cause backscattering include buried rocks, shells, internal layering and any other source of lateral variability.

A large amount of work has been reported studying acoustic scattering from rough surfaces. There are two well-known theoretical methods for calculating acoustic scattering from rough surfaces: the Rayleigh-Rice method and the Kirchhoff method. Based on the small perturbation approximation, the Rayleigh-Rice method treats the rough surface as a perturbation to a smooth plane and the resulting scattering coefficient due to the presence of roughness is calculated. The Kirchhoff approximation approach is more pertinent to large-scale roughness, and the scattering surface is assumed to be sufficiently smooth so that the tangent plane at any point of the surface determines the reflection properties. Some efforts are also put into combining the above two methods in a composite model, treating small-scale roughness by the Rayleigh-Rice method and large-scale roughness by the Kirchhoff method [1]. A good review of theoretical work and experimental investigations on rough surface scattering can be found in Ogilvy's book [2].

At low frequencies and in regions where significant bottom penetration exists, such 
as thickly covered sediment areas, scattering caused by inhomogeneities within the sediment volume can be the dominant influence on bottom backscattering. However, compared with the reported work on rough surface scattering, only limited results are available for studies of scattering from within the bottom. The modeling of bottom backscattering becomes more and more complicated by including more physical processes in order to understand the scattering mechanisms and predict the scattering strength. Nevertheless, some common phenomena observed in experiments still cannot be explained by the existing models. As for typical scattering experiments, the source and receiver are often far away from the bottom, in which case a large bottom area is ensonified, or only a single source/single receiver configuration is used so that the received signals are the superposition of the scattered returns from all directions. Both of these situations are unfavorable for bottom backscattering investigations. They may result in artifacts for the data analysis and even lead to misunderstanding of the scattering process. Therefore, a scattering experiment which uses a directional source and/or receivers suspended near the ocean bottom is beneficial for bottom backscattering studies.

As part of the Office of Naval Research Bottom/Subbottom Acoustic Reverberation Special Research Program (ARSRP), a backscattering experiment was conducted over a sediment pond on the western flank of the Mid-Atlantic Ridge in July 1993. A chirp source and vertical line array were deep-towed only $200 \mathrm{~m}$ to $400 \mathrm{~m}$ above the seafloor, while the sediment thickness was up to $400 \mathrm{~m}$ in the middle of the sediment pond. For a transmitted acoustic source level of approximately $200 \mathrm{~dB}$ re: $\mu \mathrm{Pa} @ 1$ $\mathrm{m}$ and a source frequency of $250-650 \mathrm{~Hz}$, the acoustic signal can penetrate to the rock basement beneath the sediment layer in spite of sediment attenuation and geometrical spreading. Since the water-bottom interface was flat, this experiment provided a good opportunity to investigate backscattering due to inhomogenieties beneath the water-bottom interface.

The objectives of the subsequent experimental data analysis were: 
- To detect the sediment structure. This subbottom profiling can be obtained using the beamformed output in the endfire (normal incidence) direction. It provides information about geological features, such as horizontal variations and heterogeneous distributions of materials, which could be the sources of the backscattering.

- To study random scattering in the normal incidence direction. The normal incidence direction returns as the output of the beamforming operation contain the information about coherent reflection and incoherent scattering. By removing the coherent part of the signals, the random scattering can then be revealed.

- To investigate the backscattering in oblique directions. A multiple constraints beamforming technique along with a subtraction procedure is applied here in order to reduce the contamination of oblique backscattered returns by normal incidence returns. The locations of the major scatterers are investigated and the backscattering strength versus grazing angles is calculated.

- To relate the scattering in the normal incidence and oblique directions to the geological features identified in sediment profiling. This can be of benefit to the future modeling work and to the understanding of the scattering mechanisms. Hopefully, it can offer the possibility of locating the major scatterers within the sediment by subbottom profiling.

\subsection{Relevant Literature Review}

Since this research involves several different areas, the purpose of this section is to provide a brief review of the references which have direct relevance to each area.

\subsubsection{Volume Backscattering Models}

It is generally accepted that backscattering from sediment volume inhomogeneities can contribute significantly to seafloor backscattering, especially at low frequencies, 
small grazing angles and where the bottom is flat. From the 1960's, a number of models have been developed in order to examine the underlying scattering mechanisms and to predict the strength of the backscattered signals.

Stockhausen [3], as early as 1963, derived a volumetric backscattering strength expression assuming that the water-sediment interface is flat and refracting (with the consequent critical angle effect), and with the homogeneous sediment containing a uniform set of solid spherical particles which act to scatter the acoustic energy. Treating the small spheres as uncorrelated point scatterers, he employed Morse's expression [4] which is valid for scattering from spheres much smaller than a wavelength. In his model, Stockhausen represented all the scattering processes by a single volume backscattering cross section without further exploring any physical mechanisms.

Nolle et al. [5] at almost the same time developed their own model. They described the scattering from the sediment volume based on a random distribution of scattering amplitude per unit volume. The scattering strength distribution was considered to be centered at a mean value with random deviations. For simplicity, the scattering autocorrelation function was assumed to have the form of an exponential decay. The autocorrelation length was proportional to the particle size in the sediment volume, thus going one step further compared to Stockhausen's uncorrelated point scatterer model. Nolle et al. also conducted an experiment in the laboratory studying acoustic scattering from a smooth sand surface and compared their model with the collected data. It showed that the model had some difficulties in explaining the scattering from sub-critical angles.

Crowther [6] included both interface roughness and volume inhomogeneity effects in his ocean bottom backscattering model. Kuo's formula [7] for backscattering from the rough interface between two homogeneous fluids was used here. Extending Nolle et al.'s model, he assumed the correlation function for impedance fluctuations to be elliptic exponential, which was able to account for the anisotropy of the scatterers. 
However, this model also had problems with scattering at sub-critical angles when comparison with Nolle et al.'s laboratory backscattering data was made. Without knowledge of the detailed shape of the correlation function, it would be inappropriate to predict such characteristics as the frequency dependence of the scattering employing this model.

There were some singular features of the backscattering coefficient obtained in experimental measurements at sea [8] [9]. These included the frequency independence (or weak frequency dependence) over the frequency range 1-100 KHz and an angular dependence proportional to $\sin \theta$ for grazing angles $\theta$ from 5 to 50 degrees. In order to interpret this, Ivakin and Lysanov [10] proposed their statistical models on the basis of the geoacoustical model presented earlier by Lysanov [11]. The scattering was thought to come from "sharply anisotropic random inhomogeneities (fluctuations of the refractive index): large-scale in the horizontal plane and small-scale in depth" in the sediment. They used the Born approximation to derive an expression for the equivalent surface scattering coefficient, which was not dependent on the frequency if the absorption coefficient was proportional to frequency and the power spectrum of the inhomogeneities was inverse proportional to the wavenumber to the third order. Also, they extended the single-scale (horizontal correlation coefficient) model to two-scale and multi-scale models so as to account for the variability of the horizontal correlation coefficient in different regions. The interface effect was ignored by assuming that the change of sound speed and density from the water to the water-saturated sediment were very small. The model prediction agreed very well with the data after the model/data fit of a free parameter: the ratio of the vertical and horizontal correlation length.

In their next paper [12], Ivakin and Lysanov revised their model to account for the interface roughness effect. The Kirchhoff approximation was applied to the rough interface backscattering case. The model studied in detail the influence of interface roughness on the volume backscattering cross section: for slow or nonreflecting bot- 
toms, the volume scattering did not depend on whether the bottom interface was smooth or not; for fast bottoms, the roughness effect was significant in the subcritical grazing angle region. Physically, the reason for the latter result was because the rough interface would enhance the penetration of sound into the bottom medium at small grazing angles and therefore intensify the scattering of sound by volume inhomogeneities. They emphasized the nonadditiveness of the scattering effects due to volume inhomogeneities and water-bottom interface roughness, which was different from several other researchers who assumed that these two effects were uncorrelated. The authors did not include the possible lateral wave effects.

Ivakin [13] extended his model for scattering from volume inhomogeneities again to deal with a stratified bottom. The correlation function for the random spatial fluctuations of the acoustical parameters was considered to be "statistically homogeneous in the horizontal plane and quasi-homogeneous with respect to the vertical coordinate". The model allowed considerable changes in the sound speed and the density with depth. He studied the linear increase in sound speed and density case and the linear decrease in sound speed case. The results showed good model/data agreement.

For the high-frequency $(10-100 \mathrm{kHz})$ bottom backscattering model proposed by Jackson et al. [1] in 1986, the composite roughness approximation was applied for the scattering due to interface roughness. To include the volume scattering contribution, they employed Stockhausen's formula [3] and accounted for the volume scattering by an equivalent surface backscattering coefficient. The assumption inherent in Jackson et al.'s approach was the neglect of any correlation between the scattering due to interface roughness and sediment inhomogeneities, which was different from Ivakin and Lysanov's model [12]. Multiple scattering was also ignored. The comparison of the model with two sets of data suggested that "in soft sediment, sediment volume scattering is likely to be more important than roughness scattering, except near normal incidence and for grazing angles smaller than the critical angle. For sand bottoms, 
roughness scattering is relatively more important". However, the volume scattering parameter was still obtained from model/data fits without any relationship to the sediment properties.

Mourad and Jackson [14] generalized Jackson et al.'s model [1] by including the sediment sound absorption in the interface boundary condition. They constructed some empirical relationships for estimating surficial values of the input geoacoustic parameters to the model using bulk measurements of logarithmic grain size $M_{z}$ proposed by Hamilton [15]. These parameters otherwise could only be obtained by direct measurement, which was not available for many datasets. Nevertheless, as they pointed out, the gradients of sound speed and density, which might play an important role in affecting the acoustical response of the sediment, were not included in this model.

Later, Mourad and Jackson [16] went one step further by including the gradient of sound speed in their low-frequency $(100-1000 \mathrm{~Hz})$ model. The volume scattering model was very similar to Ivakin's model [13] and they assumed "a distribution of uncorrelated omnidirectional point scatterers in the sediment causes the backscattering of sound". The model related the oscillation of the backscattering strength to the acoustic field within the sediment and suggested that the total backscattering from the ocean bottom was controlled by the processes affecting sediment volume scattering, for example strong layering. They also discussed the possible errors in the measurement of bottom scattering strength near the normal incidence direction using omnidirectional sources and receivers. For low-frequency backscattering, the questions for this model were how good the local plane-wave assumption inherent in the model was and the validity of attributing the volume scattering effect to an equivalent surface scattering process.

Hines [17] developed a backscattering model in an approach similar to Ivakin's [13]. He followed Chernov's work [18] and applied the Born approximation and the 
far-field assumption. Backscattering of the acoustic energy in his model was due to the sound speed and density fluctuation in the sediment, which he related to the fluctuation of porosity. The lateral wave effect was for the first time included in this model, attempting to interpret the phenomena observed in experiments at small grazing angles. An exponential decay correlation function was assumed and the model fit several published data very well. Yet a priori knowledge of the correlation function and variance of the porosity were needed for the model prediction. The approach of decomposing the incident spherical wave into a refracting plane wave and an evanescent wave was debatable.

Tang in his thesis [19] and Tang and Frisk in their several papers [20] [21] [22] discussed in detail the scattering from a random layer or half-space where the sound speed is assumed to be a constant plus a small random component. An integrodifferential equation method was applied. An interesting point was that the spatial correlation length of the scattered field could be used to infer the correlation length of the scatterers. This provided a way of inverting for the bottom parameters critical to bottom scattering by measuring the scattered field using multiple receivers. Also taken into consideration was the anisotropy of the scatterers. Besides examining the combination of interface roughness and volume inhomogeneity effects, an attempt was made to solve the near-field problem in low-frequency scattering when the far-field assumption was not appropriate anymore.

Recently, Lyons et al. [23] extended Jackson et al.'s model [1] for scattering from the seafloor. In addition to the composite roughness model for interface scattering and Stockhausen's expression for volume scattering [3], they included the volume scattering from a random inhomogeneous continuum and scattering from subbottom interfaces. Their approach to calculating the scattering from the random inhomogeneous continuum was similar to Hines' work [17]. The compressibility and density variations were modeled. The correlation function could have different correlation lengths in the horizontal and in the vertical to allow for anisotropy in the volume 
scattering model. They employed the Born approximation to obtain the volume backscattering cross section, which was a free parameter in Jackson et al.'s model, before fitting it to Stockhausen's formula for an effective surface backscattering coefficient. They estimated all of the input parameters except the horizontal correlation length from core data. The comparison with data was very good. Because this model was more complicated and had more input parameters, its performance depended very much on the estimation of those parameters.

For elastic media, in addition to compressional waves, shear waves and evanescent waves (e.g., Scholte waves at fluid-elastic interface) may play important roles in bottom scattering. Their interactions generate some unique scattering features that cannot be observed in fluid-fluid environment [24] [25] [26] [27]. For discrete elastic targets in a layered waveguide, the resultant scattering is treated by the transition matrix formalism [28] [29] or the boundary element method (BEM) [30]. However, the elasticity effect will not be considered in this thesis work.

\subsubsection{Experimental Data Analysis}

Since World War II, many acoustic backscattering experiments have been carried out both in the laboratory and at sea. Generally, the experimental data analysis shows an angular and frequency dependence of the scattered signals as well as the effects of different bottom types.

Early investigations of bottom backscattering used an omnidirectional source and receiver. Mackenzie [31] presented the first deep-sea measurement results in 1961. The source and receiver were close to the sea surface. He found that the bottom backscattering strength obeyed Lambert's law of diffuse reflection for grazing angles from 30 to 90 degrees, with the scattering constant (Mackenzie coefficient) having a value of $-27 \mathrm{~dB}$. This value didn't change for the frequencies $530 \mathrm{~Hz}$ and $1030 \mathrm{~Hz}$. In 1968, Merklinger [32] reported his experiment using a hydrophone and explosive 
charges suspended near the ocean bottom. The data analysis indicated that the reverberation due to the subbottom layer structure contributed significantly to the total bottom reverberation. This highlighted the importance of subbottom inhomogeneities in the prediction of the bottom backscattered field.

Bunchuk and Zhitkovskii [33] later gave an overview of bottom scattering in shallow water regions. The definition of the scattering strength and several experimental methods for bottom scattering data acquisition were discussed. Based on a review of the published experimental results in the frequency range $10-100 \mathrm{kHz}$, they pointed out the differences between the scattering properties in shallow and deep water regions. In shallow water regions the inhomogeneities dominated the bottom scattering and in deep water regions the rough interfaces dominated. As they suggested, it is necessary to include both the interface roughness and volume inhomogeneities in the bottom scattering models.

There is one thing that needs to be argued in the conventional method of estimating the bottom backscattering strength from experimental data. For single source and single receiver configuration, usually all the returns are assumed to come from the bottom interface, which is questionable if the scatterers within the bottom are also the major contributors to the bottom reverberation. The scattered returns from the interface and from within the volume cannot be separated based on arrival time, especially at small grazing angles. As the result, the inferred angular dependence of the backscattering strength may be erroneous.

Thanks to the evolution of technology, later experiments have used the directional sources and/or receivers to obtain scattering data from the ocean bottom. Boehme et al. [34] conducted an experiment to study backscattering at low grazing angles in the frequency range $30-95 \mathrm{kHz}$, and found that the Lambert's law fit for grazing angles ranging from 2 to 10 degrees. Preston and Akal [35] employed a towed horizontal array and a suspended vertical array to measure ocean basin reverberation. They 
found that strong backscattering regions were related to basin topographic features. Hines and Barry [36] carried out an experiment at the Sohm Abyssal Plain with the source and receiver suspended close to the smooth seabed. Their acoustic array contained an omnidirectional hydrophone and a ring projector with vertical directivity. Different features were observed for backscattering from different bottom types.

The above describes some of the typical experiments that have been conducted. Since scattering from the ocean bottom is a very complicated process and has great variability from region to region, our understanding of it is still very limited. Together with further theoretical modeling, more experiments are expected to be carried out in order to test the models and clarify the physical mechanisms associated with the bottom scattering process.

\subsection{Outline of Thesis}

In Chapter 2, a detailed description of the experiment is given first. Several methods which will be used in the data analysis are then introduced. Among them are the Array Element Localization algorithm and the method for calculating the bottom backscattering strength. Conventional single-constraint beamforming and multiple constraints beamforming are discussed in detail. The latter is used in the next chapter to discriminate between signals scattered in desired directions and those scattered in other directions. Simulations are also performed to prove the effectiveness of the multiple constraints beamforming technique along with a subtraction procedure in rejecting the strong signals coherently reflected by sediment layering in the normal incidence direction.

Chapter 3 is devoted to the backscattering experiment data analysis. The preprocessing of the experimental data is first discussed. The focus of this chapter is on the analysis of backscattering data from the normal incidence and oblique directions. It 
is found from the subbottom profiling that the sediment is dominated by a layered structure. Two identified irregular regions within the sediment volume have been demonstrated to be the major sources for backscattering in both the normal incidence and oblique directions. The backscattering strength as a function of grazing angle is also calculated.

Chapter 4 concludes the research in this thesis and addresses future work related to this research. 


\section{Chapter 2}

\section{Experiment Description and}

\section{Theory}

\subsection{Experiment Description}

On July 24-26, 1993, as part of the Acoustic Reverberation Special Research Program (ARSRP), a bottom backscattering experiment was conducted over the site A sediment pond located at $26^{\circ} 11^{\prime} \mathrm{N}, 46^{\circ} 09^{\prime} \mathrm{W}$ in the vicinity of the Mid-Atlantic Ridge [37].

The water depth was about 4400 meters for most of the sediment pond area and the sound speed profile was a typical linear deep ocean profile obtained from the XBT measurements. The gradient was $0.0154 / \mathrm{s}$ for the water column close to the seafloor. The sound speed at the water-sediment interface was extrapolated to be $1530 \mathrm{~m} / \mathrm{s}$.

The oval-shaped sediment pond was approximately $15 \mathrm{~km} \mathrm{x} 20 \mathrm{~km}$ in size. The thickness of the sediment cover varied from almost no cover at the edge of the pond to about 430 meters in the middle of the pond. For areas where the backscattered data were collected, the sediment was at least $100 \mathrm{~m}$ thick. The top layer beneath the water-sediment interface was made of calcareous mud with a sound speed of 1510 $\mathrm{m} / \mathrm{s}$ according to core data [38]. No accurate information was available about the attenuation coefficient or the sound speed gradient in the sediment area where the 
experiment was conducted. A deep towed high-frequency chirp sonar and in situ optical survey revealed that the seafloor was virtually flat with rms roughness less than $10 \mathrm{~cm}$ [39]. A rough basalt basement was beneath the sediment layer.

Figure 2.1 shows the bathymetry of the sediment pond and the ship track along which the experiment was carried out. For the results presented in this chapter, we will only analyze the data collected along the straight line shown in the figure. Figure 2.2 depicts the experimental scenario. The acoustic system was deployed at the west side of the pond and recovered in the middle after being towed across the entire sediment pond.

The acoustic source used in the experiment is part of the Deep Towed Acoustics/Geophysics System (DTAGS) and was developed by the Naval Research Laboratory - Stennis Space Center[40]. The source signal is a linear frequency-modulated (LFM) upchirp signal in the frequency range $250-650 \mathrm{~Hz}$. The designed source level is $200 \mathrm{~dB}$ re: $\mu \mathrm{Pa} @ 1 \mathrm{~m}$. The duration of the source signal is $0.125 \mathrm{~s}$. The receiver is a 24 element vertical line array(VLA) hung beneath the source, with a spacing between adjacent hydrophone elements of $2 \mathrm{~m}$. The cable connecting the source and the top hydrophone is $57.5 \mathrm{~m}$ long. A weight is attached to the end of the array to keep it close to a vertical configuration. Figure 2.3 shows the geometry of the acoustic transmitting and receiving system. The source signal is transmitted once every 32 $\mathrm{s}$. For each ping, the hydrophones record $4.5 \mathrm{~s}$ of waveforms starting $0.1 \mathrm{~s}$ before the source trigger. The sampling interval is $0.432 \mathrm{~ms}$. More than 5000 pings were transmitted throughout the experiment. Figure 2.4 shows the received signal and its spectrum at one hydrophone for one ping.

During the experiment, the DTAGS source was towed at a depth of about 200400 meters above the seafloor, while the ship used the high-resolution (P-code) GPS dynamic positioning to maintain a constant speed over ground (SOG) of 0.5 knot, with several stops to ensure that the array returned to nearly vertical alignment. 


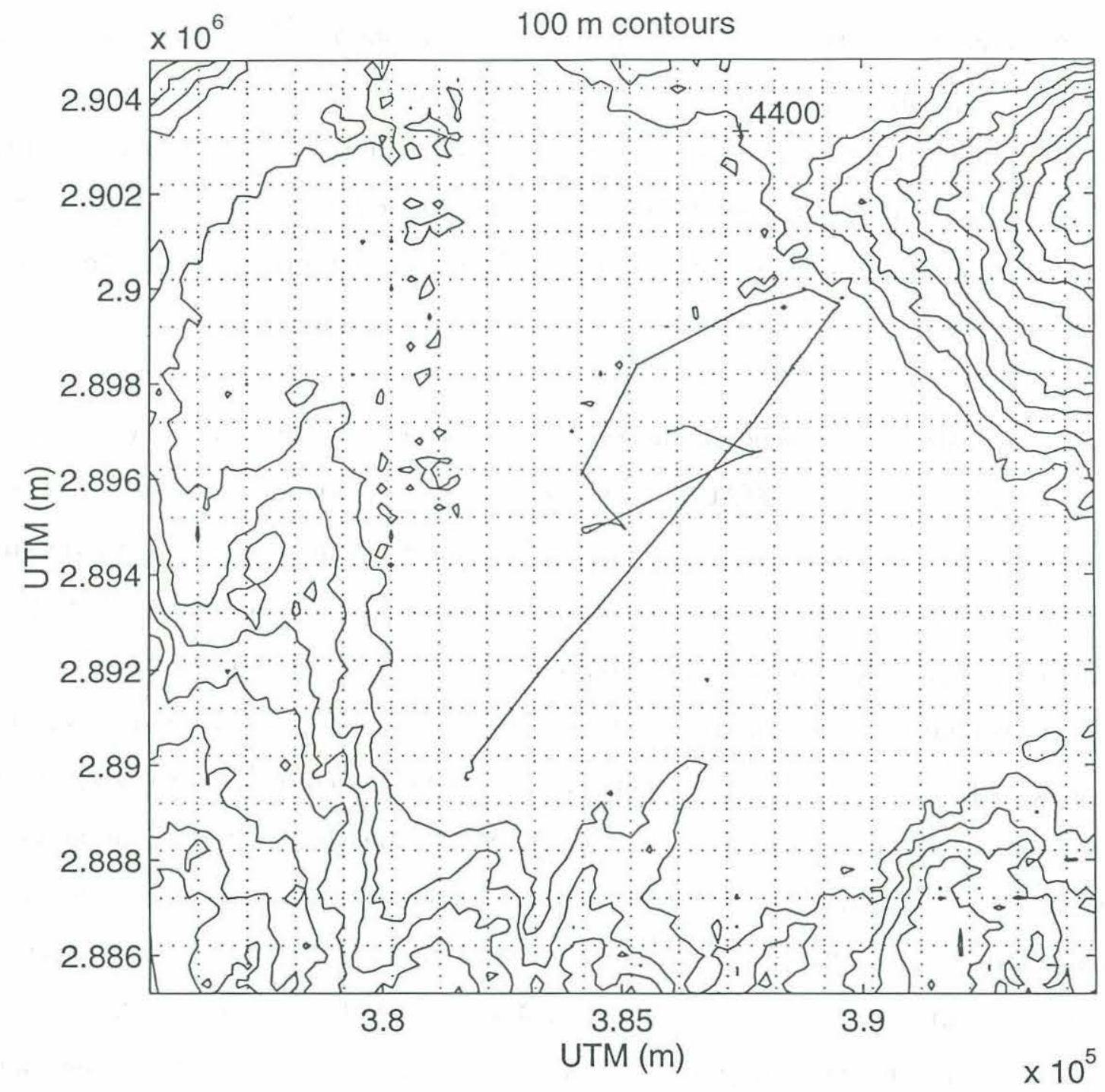

Figure 2-1: Bathymetry and ship track in the ARSRP Site A sediment pond. 


\section{ARSRP Sediment Scatter Experiment \\ R/V Knorr, July 24 - 26, 1993 \\ $26^{\circ} 11^{\prime} \mathrm{N} \quad 46^{\circ} 09^{\prime} \mathrm{W}$}

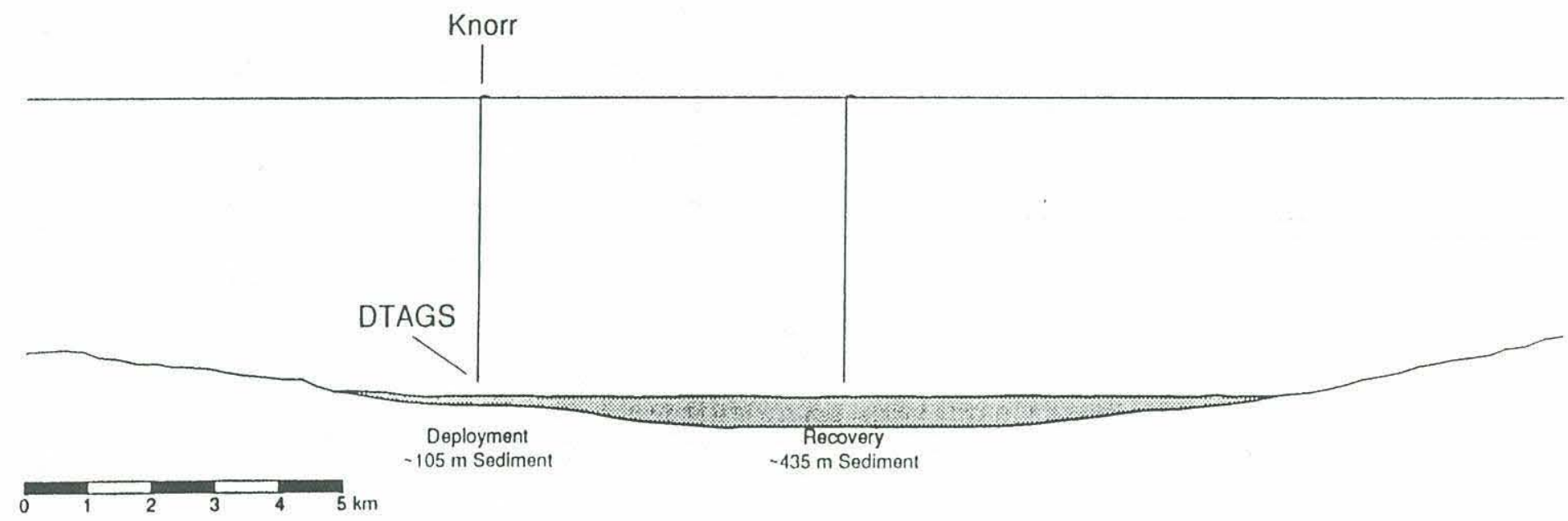

Figure 2-2: The experimental scenario. 


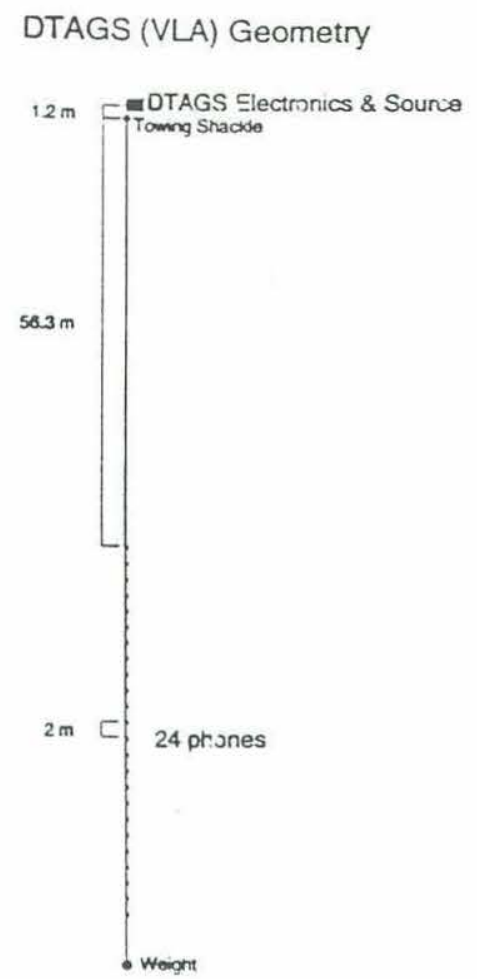

Figure 2-3: The DTAGS source and receiving array geometry.

The actual array shape is estimated by using the direct and bottom reflected arrival times on each hydrophone. The details of the array element localization method was given later in this chapter. Figure 2.5 shows the estimated array configuration for one location. It can be noticed that the array is roughly vertical. Generally it is found that the array tilt angle is within 5 degrees of vertical.

\subsection{Beamforming and Simulations}

\subsubsection{Introduction}

Beamforming is a spatial filtering process which is capable of separating signals that come from different spatial locations but have overlapping temporal frequency content[41]. It is often used to estimate or detect signals arriving from a desired direction in the presence of noise and interfering signais. 

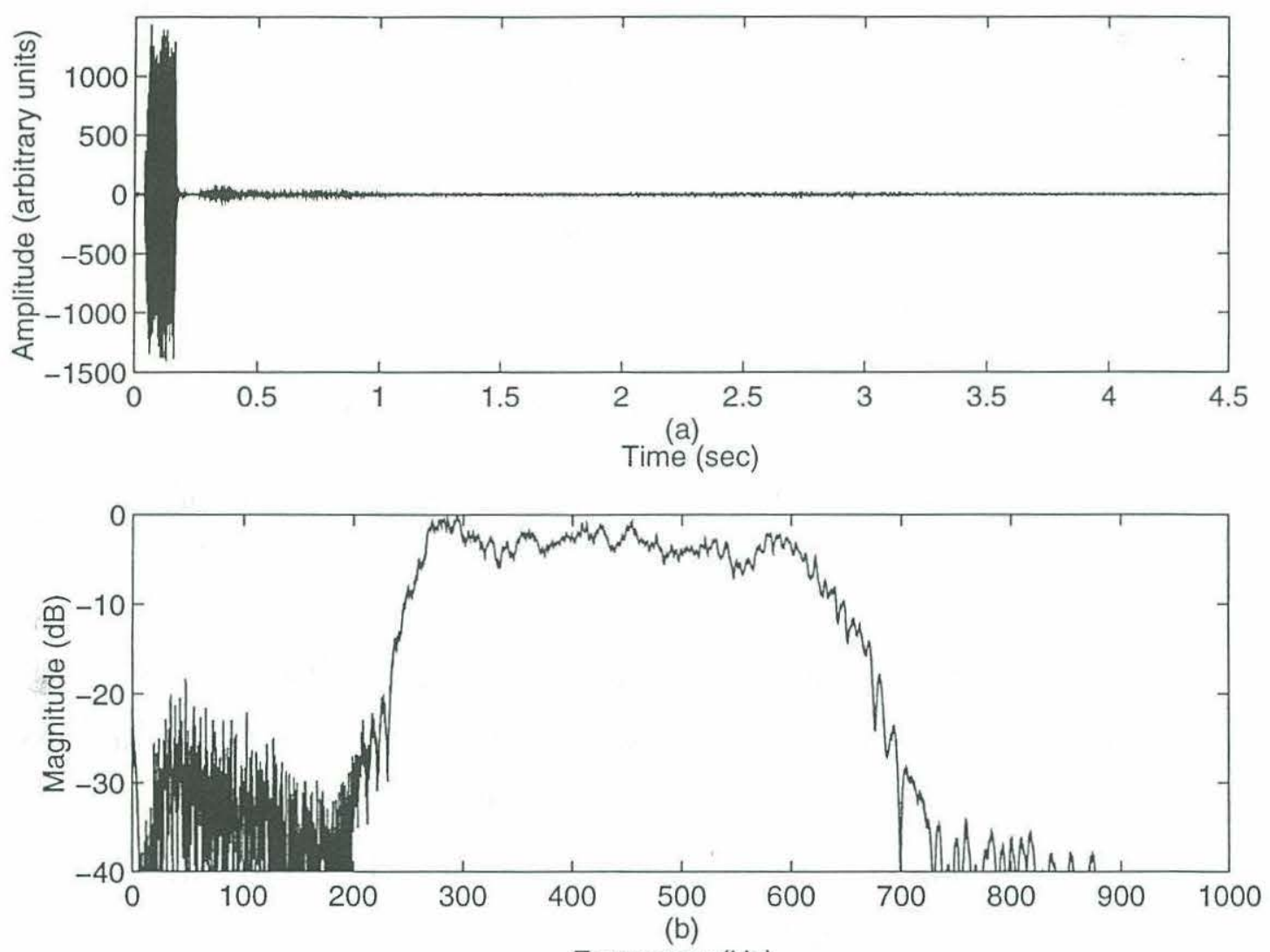

Frequency $(\mathrm{Hz})$

Figure 2-4: (a) The received signal and (b) its spectrum at one hydrophone for a typical ping 


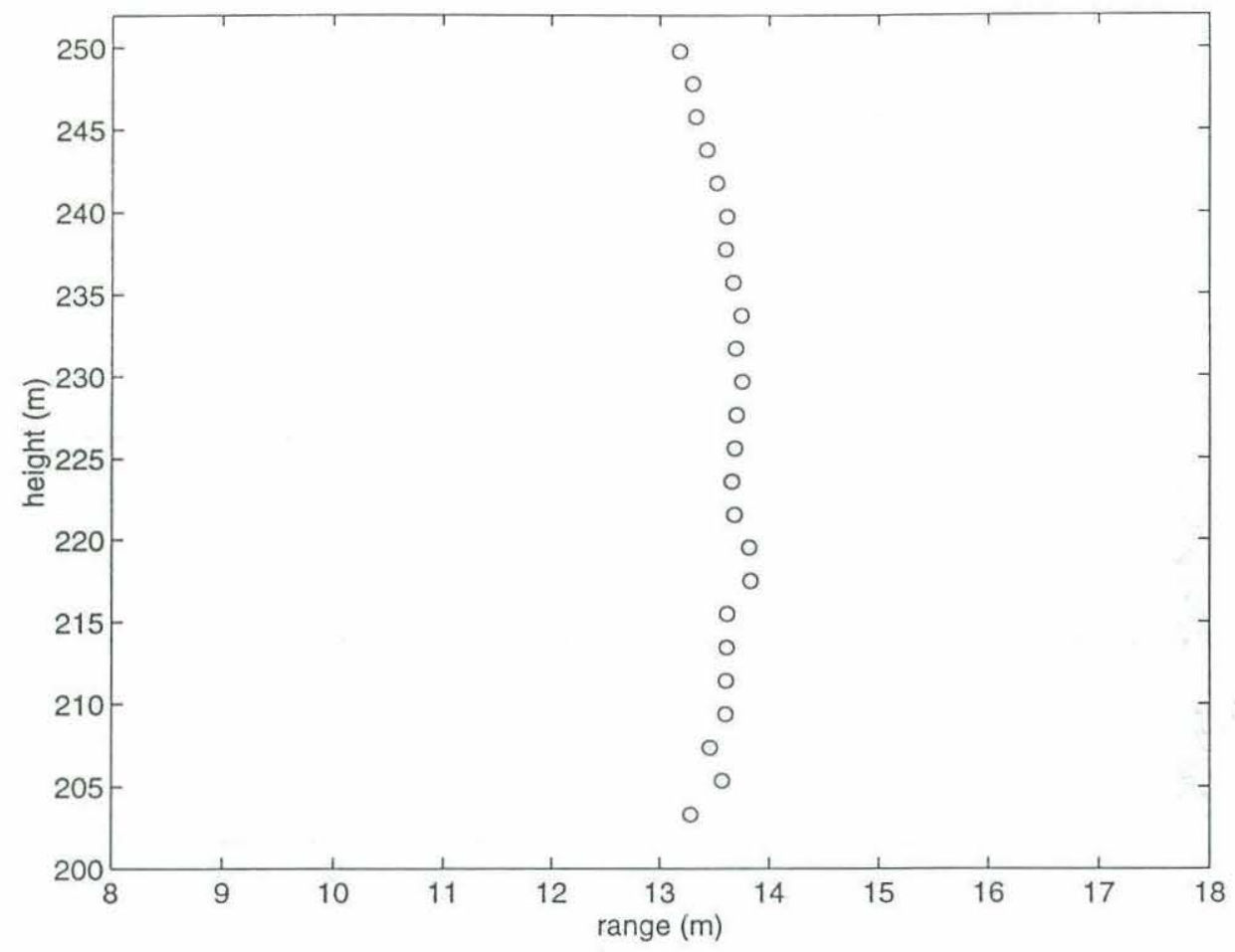

Figure 2-5: The estimated receiving array configuration for a typical ping

Generally, beamforming consists of plane-wave beamforming and matched-field processing. Plane-wave beamforming is based on the assumption that signals come from far away and propagate in free space; matched-field processing exploits the characteristics of the medium in which the wave propagates and tries to match the array data to solutions of the Helmholtz equation for a particular ocean waveguide environment. In this section, we will concentrate on plane-wave beamforming which is the tool for the backscattering data analysis in Chapter 3 .

In plane-wave beamforming, data independent linear beamforming and adaptive beamforming are two ways to extract signals from a desired direction. Although adaptive beamforming can provide high resolution and better sidelobe suppression, second order statistics must be estimated from the data (i.e., the sample covariance matrix), which is computational intensive, especially for broadband signals. Sensitiv- 
ity to environmental uncertainties poses another problem for the adaptive method on its performance. Therefore, we choose the simple and robust linear beamforming approach to process the large amount of backscattering data collected in the experiment.

The basic idea of linear beamforming is to enhance the amplitude of spatially coherent signals in the desired direction relative to background noise and directional interference (from other than the desired direction) by combining the received signals from an array of sensors with appropriate weights and time delays. Conventional linear beamforming is implemented in the time domain because of its simplicity. However, the time-domain beamformer requires the sampling rate to be much greater than the Nyquist rate so that the beam can be steered in arbitrary directions. The resultant oversampling will cause problems in computer memory and data storage. Another approach is to use frequency-domain beamforming. It accomplishes beamforming in the frequency domain by applying DFT's to the segmented sensor output, inversely transforming the resulting beam spectra and then recombining them into a time series with the overlap-add method [42]. Since during the experiment a huge data set was collected, frequency-domain beamforming will be used in the data analysis.

Many linear beamforming techniques have been developed during the last two decades. In the next part, only the constraint design of linear beamforming will be introduced.

\subsubsection{Single and Multiple Constraints Beamforming}

Linear beamforming can be viewed as shading the array by amplitude and phase factors at each hydrophone. As shown in Figure 2.6, the beamformer output at time 


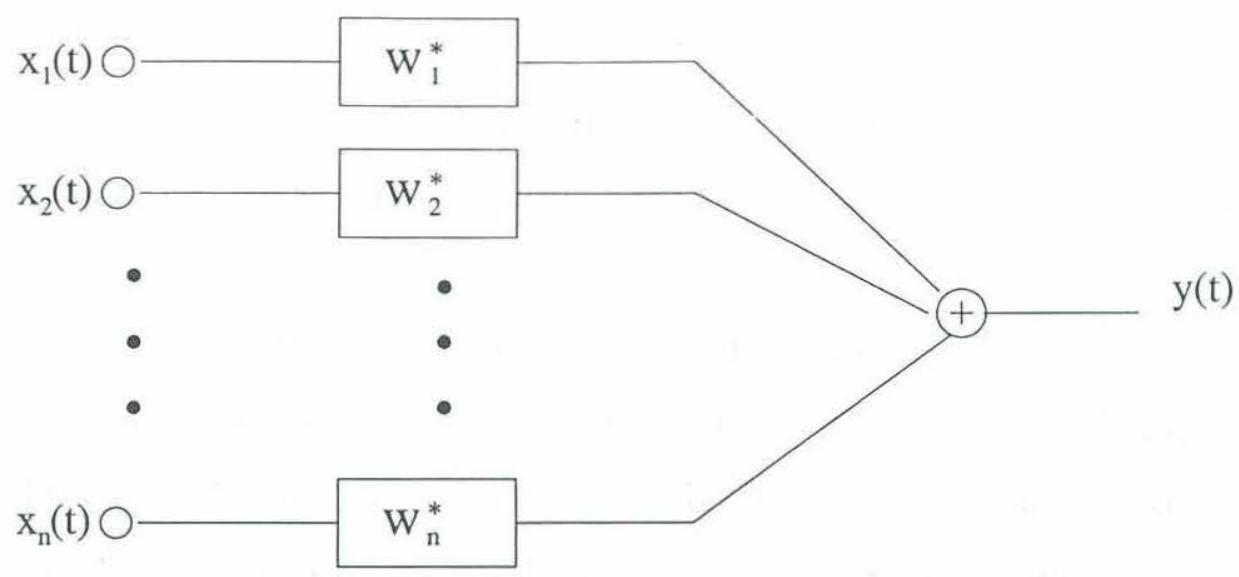

Figure 2-6: The linear beamforming scheme.

$t$, i.e., $y(t)$, is given by a linear combination of the data at $N$ array sensors at time $t$ :

$$
y(t)=\sum_{i=1}^{N} w_{i}^{*} x_{i}(t)
$$

where $w_{i}$ is the weight at receiver $i, x_{i}(t)$ is the collected time series at receiver $i$, and the ${ }^{*}$ represents complex conjugate. Notice that the weights $w_{i}$ are usually complex. An important part of linear beamforming is the design of the weights. If we use the matrix notation where boldface lower-case letters denote vectors and boldface upper-case letters denote matrices, the beamforming in the frequency domain can be expressed as follows:

$$
y(\omega)=\mathbf{w}^{\dagger}(\omega) \mathbf{x}(\omega),
$$

where $\dagger$ denotes the complex transpose operation, $\omega$ is the temporal frequency, and, in this section, we let all the vectors be columns. For broadband signals, in order to obtain the beamforming output in the time domain, frequency synthesis is necessary because the weight vector $\mathrm{w}$ is different for different frequency components.

Figure 2.7 shows the geometry of a vertical equi-spaced line array. For a plane 


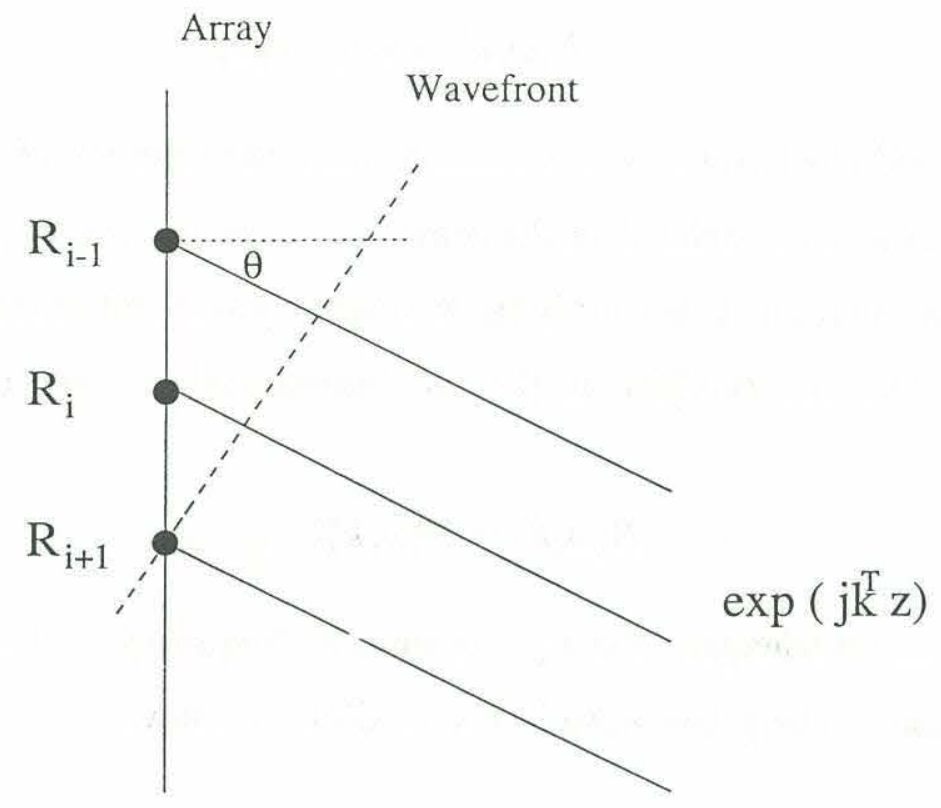

Figure 2-7: The geometry of a vertical line array.

wave, if we choose the complex exponential basis function, it has the form:

$$
x(t, z)=e^{j\left(k^{T} z-\omega t\right)},
$$

where $k$ is the wavenumber vector, $z$ is the sensor location vector, and T denotes transpose operation. For simplicity, we consider $k$ and $z$ to be in one dimension only. The response of the array processor to a plane wave is

$$
y(t, k)=\mathbf{w}^{\dagger}(\omega) \mathbf{s}(k) e^{-j \omega t},
$$

where

$$
\mathrm{s}(k)=\left[\begin{array}{c}
e^{j k^{T} z_{1}} \\
e^{j k^{T} z_{2}} \\
\vdots \\
e^{j k^{T} z_{N}}
\end{array}\right]
$$

We can suppress the time dependence $e^{-j \omega t}$, and the spatial processing then involves 
only the term

$$
F(\omega, k)=\mathrm{w}^{\dagger}(\omega) \mathrm{s}(k),
$$

which is termed the frequency wavenumber response function of an array. The beampattern for an array is defined in the context of a plane wave propagating in a locally homogeneous medium. It is simply the frequency wavenumber response function evaluated versus the direction[43]. In the one-dimensional case as shown in Figure 2.7, it is

$$
B(\omega, \theta)=\left.F(\omega, k)\right|_{k=\frac{2 \pi}{\lambda} \sin (\theta)},
$$

where $\lambda$ is the wavelength of the plane wave at frequency $\omega$. Letting $c$ be the speed of propagation of the plane wave in the medium, we have

$$
\lambda=\frac{\omega}{2 \pi c} .
$$

As to the constraint design of a beamformer, we want the signals coming from the desired direction (look direction) $\theta_{s}$ to pass through undistorted, i.e., we have the constraint

$$
\mathbf{w}^{\dagger} \mathbf{s}\left(k_{\theta_{s}}\right)=1
$$

where $k_{\theta_{s}}$ is the correponding wavenumber for direction $\theta_{s}$. In order to suppress the sidelobes in some directions (e.g., $\theta_{1}$ ) against the strong directional interferences, we can have other constraints like

$$
\mathrm{w}^{\dagger} \mathbf{s}\left(k_{\theta_{1}}\right)=\epsilon
$$

where $\epsilon$ is a small constant close to zero. The above constraints can be expressed in a matrix form as follows:

$$
\mathrm{w}^{\dagger} \mathrm{S}-\mathrm{q}=0
$$

where $\mathrm{S}$ is a matrix whose columns are the vectors $\mathrm{s}\left(k_{\theta_{1}}\right), \mathrm{s}\left(k_{\theta_{1}}\right), \ldots, \mathrm{s}\left(k_{\theta_{n}}\right)$, and $n$ is the number of constraints. q contains the values such as 1 and $\epsilon$ in Eqs. 2.9 and 2.10. Also we seek the weight vector $\mathrm{w}$ that can minimize the output of the beamformer in order to reject as much as possible background noise and interference from other 
directions. Therefore, the weight vector is chosen to minimize the functional

$$
P=\mathrm{w}^{\dagger} \mathrm{w}-\alpha\left(\mathrm{w}^{\dagger} \mathrm{S}-\mathrm{q}\right) .
$$

The first term on the right-hand side is the mean square output of the array and the second term incorporates the constraints by means of Lagrangian multiplier $\alpha$. Following the method of Lagrangian multipliers, we take the gradient with respect to the variable $\mathrm{w}^{\dagger}$ and set it equal to zero, and then obtain

$$
2 \mathrm{w}-\alpha \mathrm{S}=0,
$$

so that

$$
\mathrm{w}=\frac{\alpha \mathrm{S}}{2}=\frac{\mathrm{S} \alpha}{2} .
$$

The constraint condition gives

$$
\frac{\delta P}{\delta \alpha}=\mathrm{w}^{\dagger} \mathrm{S}-\mathrm{q}=0 .
$$

Plugging Eq. 2.14 into Eq. 2.15 yields

$$
\alpha=2\left[\mathbf{S}^{H} \mathbf{S}\right]^{-1} \mathbf{q},
$$

where $H$ represents the complex conjugate, transpose operation. Combining Eqs. 2.14 and 2.16 , we obtain the weight vector as

$$
\mathrm{w}=\mathrm{S}\left[\mathrm{S}^{H} \mathrm{~S}\right]^{-1} \mathbf{q} .
$$

Note the weight vector is data independent, which is a special case of the adaptive Multiple Constraints Matching (MCM) beamformer [44].

The conventional single constraint beamformer has only one unity constraint in the look direction as in Eq. 2.9, which is equivalent to the Bartlett beamformer. 


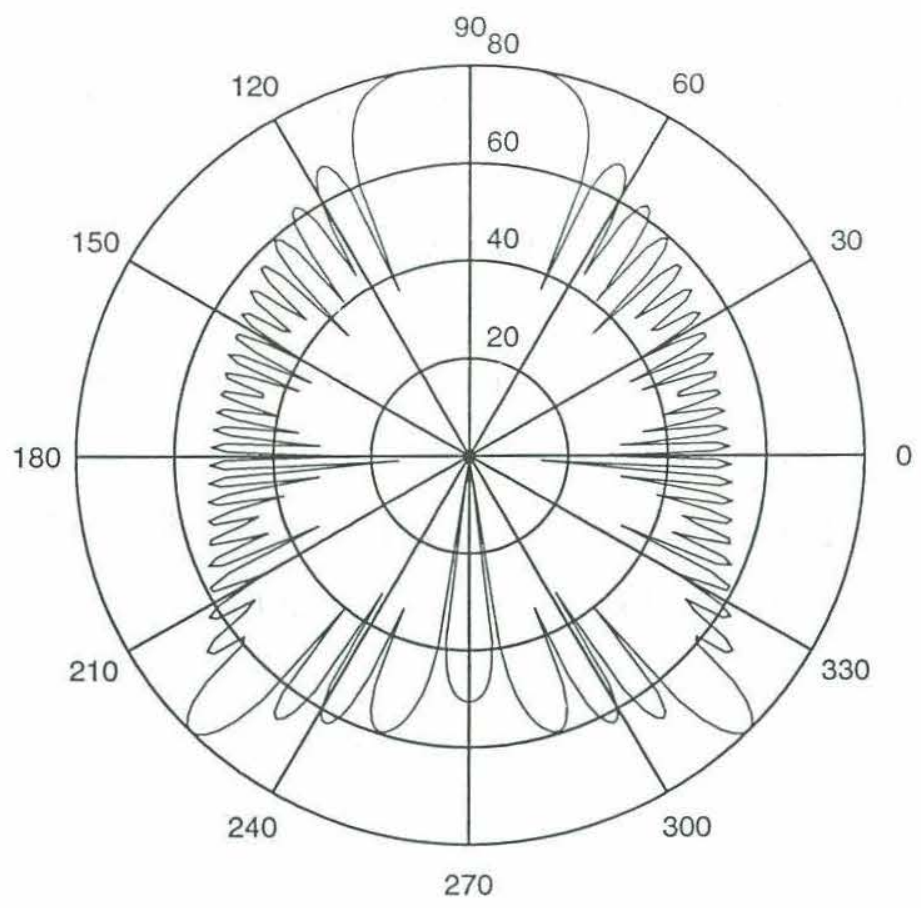

Figure 2-8: The beampattern for a single-constraint beamformer: frequency $=450$ $\mathrm{Hz}$, look direction $=-45$ degrees.

For the multiple constraints beamformer, however, a null can be constructed in a particular direction using several constraints other than the unity constraint in the look direction, to have better rejection of a directional interference. There are only $N$ (number of sensors) degrees of freedom for the system. If $n$ constraints are applied, only $N-n$ degrees of freedom can be used to minimize the mean square output of the array and account for other effects such as array bending and near field influence. Therefore, the number of constraints should be chosen carefully in order not to lose the robustness of the beamformer to environmental uncertainties.

Figures 2.8 and 2.9 depict the beampattern for a single constraint beamformer and a five-constraint beamformer, respectively. Here the frequency is $450 \mathrm{~Hz}$. The spacing between two hydrophones for the 24 element vertical line array is $2.0 \mathrm{~m}$. The look direction is -45 degrees. The array response for each circle is $20 \mathrm{~dB}$ higher than that of the neighboring inner circle. The grating lobes in the upper part of the plots are caused by undersampling. It can be seen that the sidelobe level for the five-constraint 


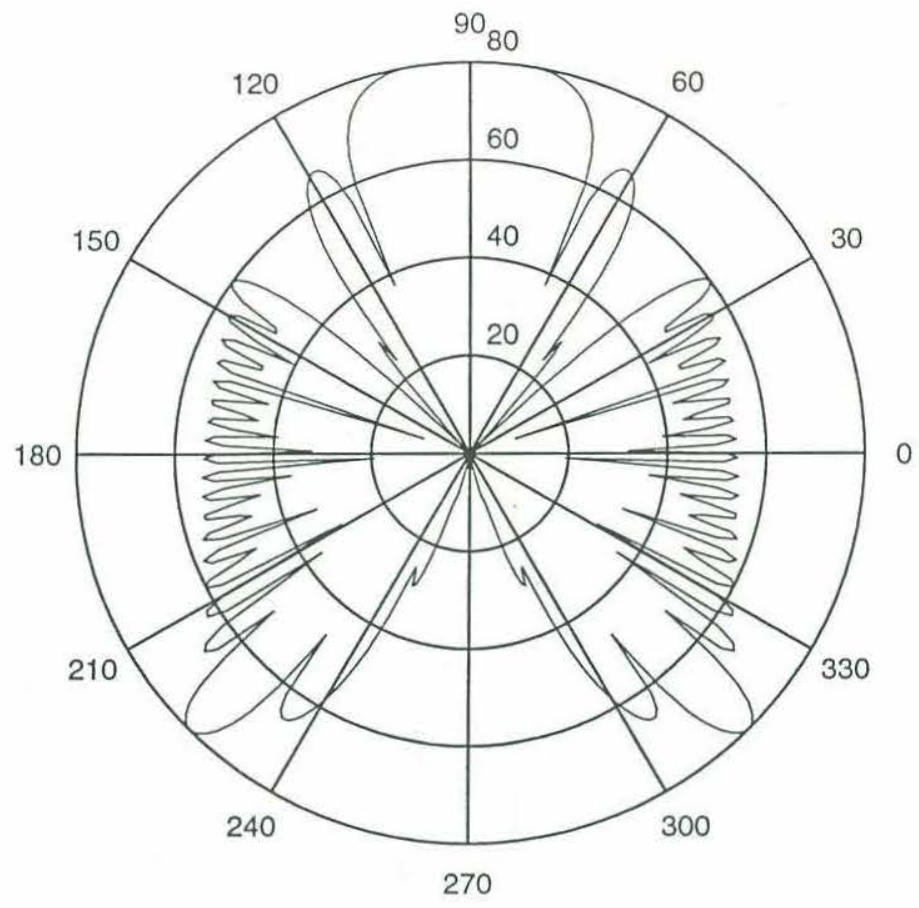

Figure 2-9: The beampattern for a five-constraint beamformer: frequency $=450 \mathrm{~Hz}$, look direction $=-45$ degrees.

beamformer in the normal and near-normal incidence directions is much lower than that for the single-constraint beamformer.

\subsubsection{Simulation}

\section{Synthetic Data Generation}

There are several uncertainties in the collected reverberation data. First, the scattering sources are numerous and their exact locations are unknown. Second, attenuation in the sediment may cause dispersion of the receiving signals. Third, knowledge of the characteristics of the background noise is unavailable. All of these factors make it difficult to evaluate the performance of the beamformer using the experimental data. Therefore, a synthetic data set has been generated to test the beamforming algorithm.

The geometry of the scatterers is depicted in Figure 2.10. For simplicity, we simulate point scatterers distributed in free space. Six scatterers are arranged on 


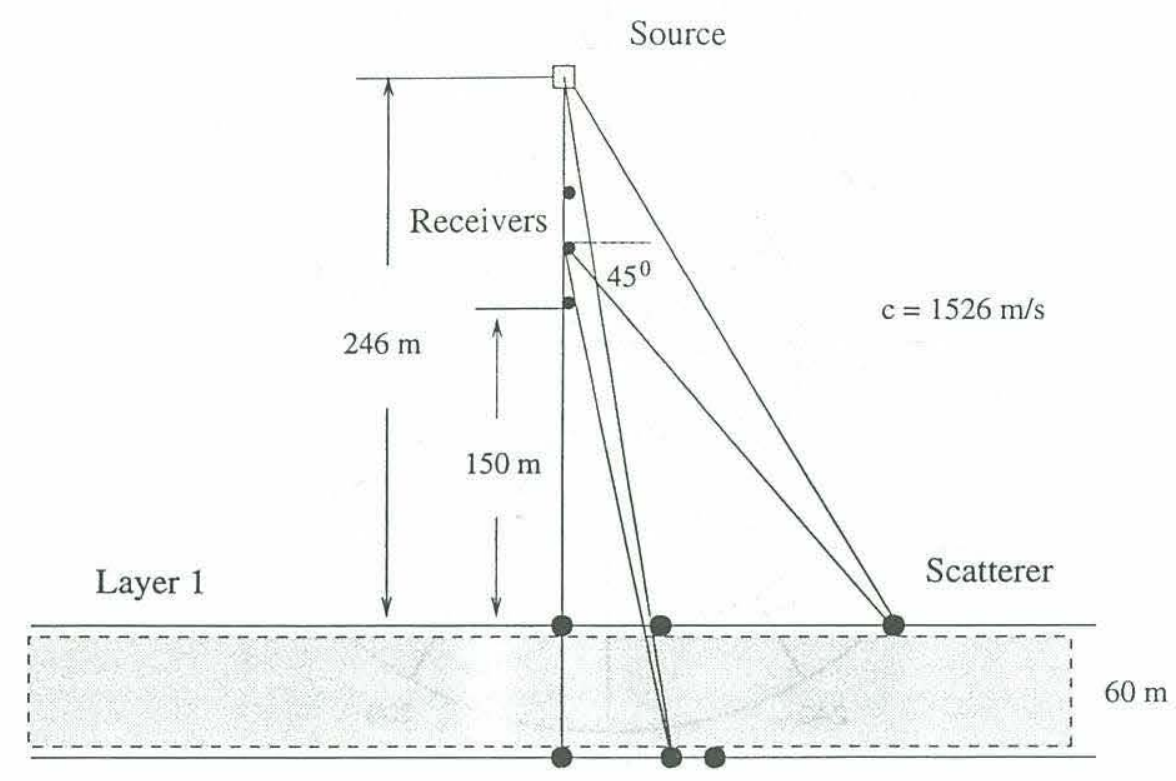

Layer 2

Figure 2-10: The geometry of scatterers.

two horizontal layers. The distance between the two layers is $60 \mathrm{~m}$. The scattered amplitudes of the two scatterers in the normal incidence direction are assigned to be ten times larger than those of the other scatterers to mimic the stronger coherently reflected returns. Here we assume that the scatterer at a 45 degree grazing angle on the first layer is the target. The three scatterers on the second layer stay in the normal and near-normal incidence directions and have almost the same arrival time as that of the target. Therefore, the resulting scattered returns cannot be separated from the target signal by time gating. The sound speed is chosen to be $1526 \mathrm{~m} / \mathrm{s}$. Spherical spreading is assumed for transmission loss. A 24 element vertical hydrophone array is suspended $150 \mathrm{~m}$ above the first layer. The spacing between adjacent hydrophones is $2 \mathrm{~m}$. The source is $50 \mathrm{~m}$ above the top hydrophone. The source signal is linear frequency-modulated (LFM), in the frequency range of 250-650 Hz. It is generated 


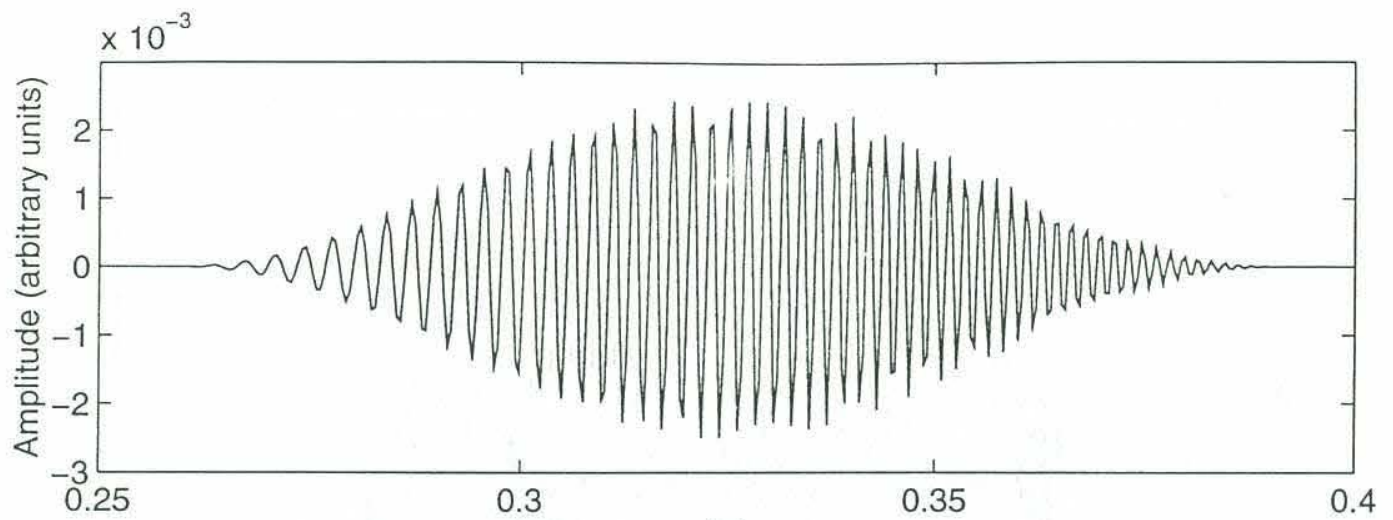

(a)

Time (sec)

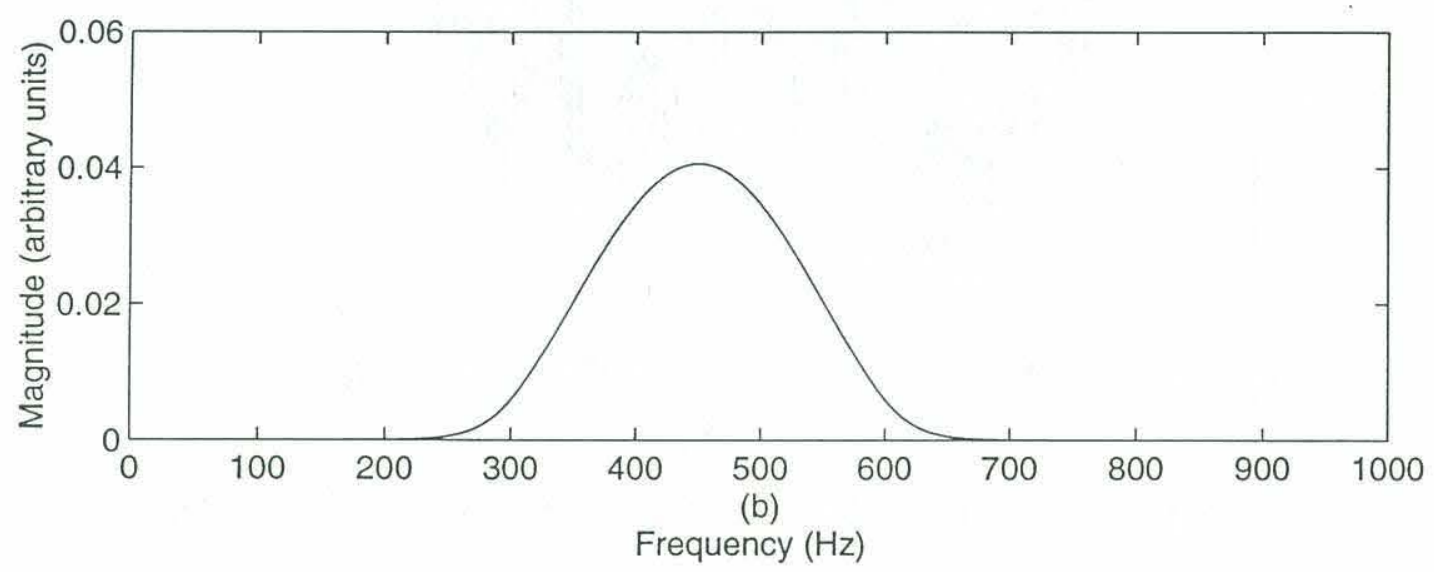

Figure 2-11: The simulated source signal: (a) the waveform; (b) the spectrum.

by the following equation:

$$
S(t)= \begin{cases}\frac{A}{2} \cos \left(2 \pi\left(f_{c} t+\frac{\mu t^{2}}{2}\right)\right)\left(1+\cos \left(\frac{2 \pi t}{T}\right)\right) & |t|<\frac{T}{2} \\ 0 & \text { otherwise }\end{cases}
$$

where $A$ is the amplitude, $f_{c}$ is the center frequency, $T$ is the pulse duration, and $\mu$ is the chirp rate. The instantaneous frequency $f(t)=f_{c}+\mu t$. The frequency is swept from $f_{c}-\mu T / 2$ to $f_{c}+\mu T / 2$ for a total bandwidth of $2 W=\mu T$. Here $f_{c}=450 \mathrm{~Hz}, T=131.3 \mathrm{~ms}$, and $\mu=3045.8 \mathrm{~Hz} / \mathrm{s}$. The waveform and the magnitude of the spectrum for the generated source signal are shown in Figure 2.11 (a) and (b). Figure 2.12 shows the simulated time series at the bottom hydrophone due to the six point scatterers. Matched filtering is also applied to the generated signals in order for the simulation to be consistent with the experimental data processing. 


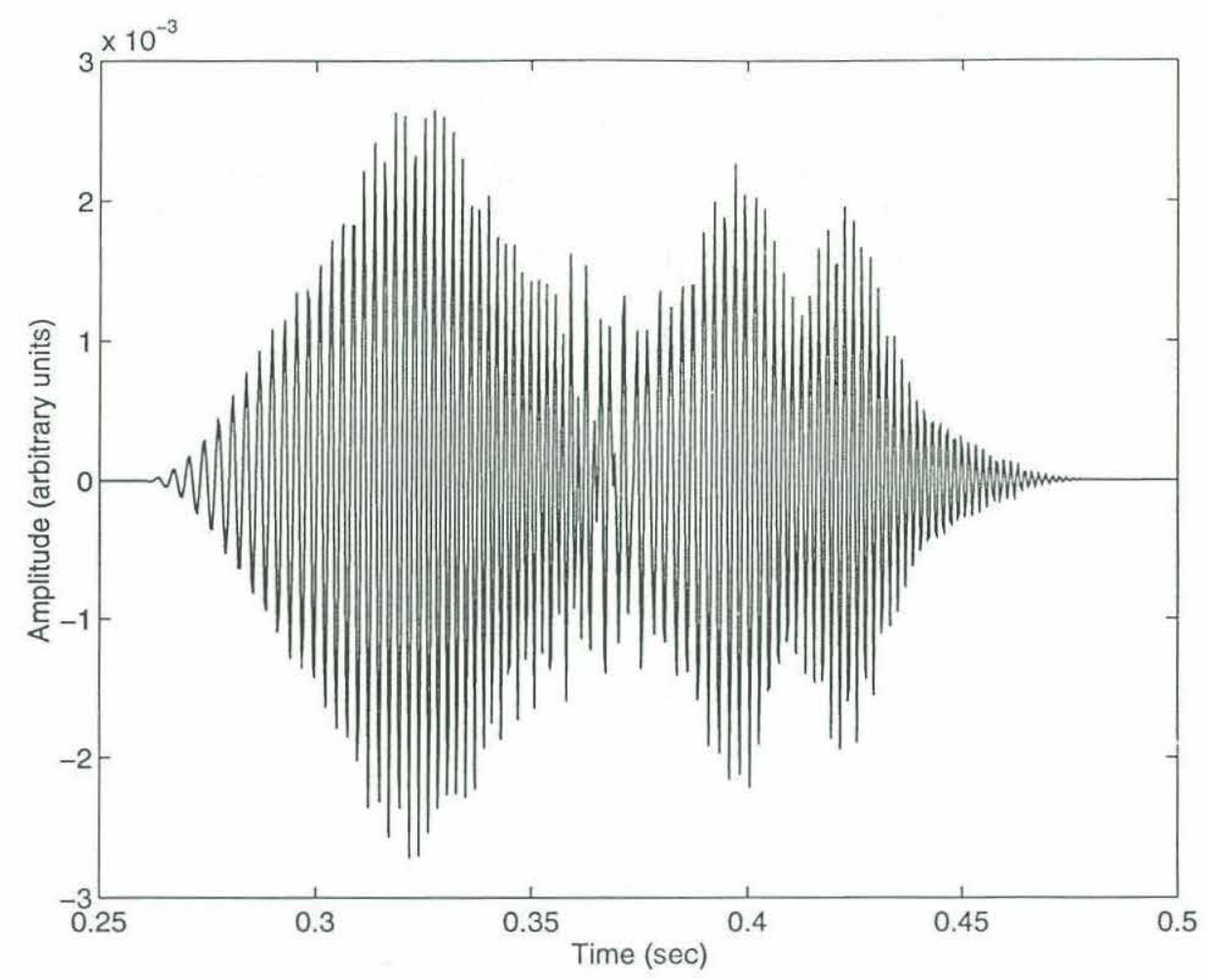

Figure 2-12: The simulated time series at the bottom hydrophone.

From now on in this section, assume that all of the signals have been matched filtered. Figure 2.13 (a) shows the returns from the target alone. Figure 2.13 (b) shows the signals from the scatterers in the second layer. Figures 2.13(c) and (d) show the signals collected at the bottom hydrophone, with different time windows. The signals from the scatterers in the second layer can be seen overlapping with the target signals.

\section{The Beamforming Results}

From the beampatterns shown in Figs. 2.8 and 2.9, it can be seen that the singleconstraint beamformer does not have sidelobe levels low enough to achieve good rejection of the unwanted returns in the normal and near normal incidence directions. However, the multiple constraints beamformer can have controllable sidelobe levels for higher attenuation and better beamforming results. This can be seen from the following simulation results. 


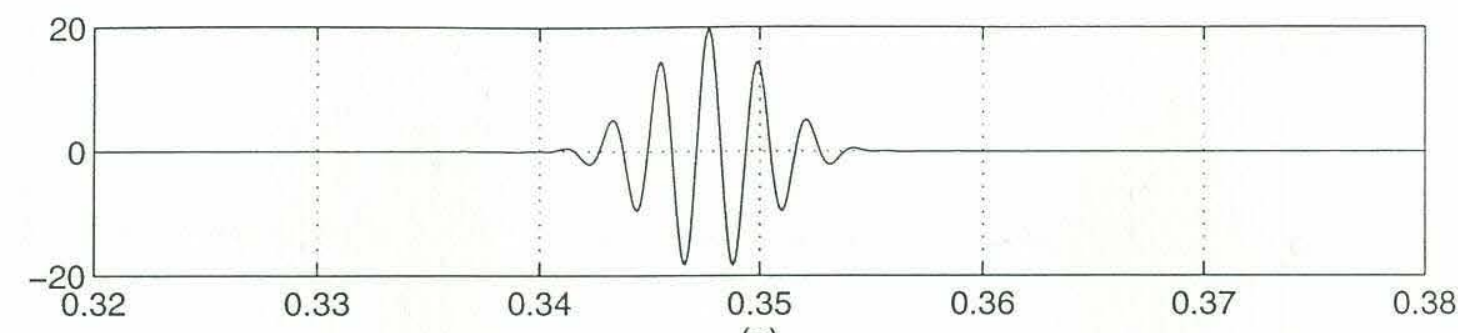

(a)

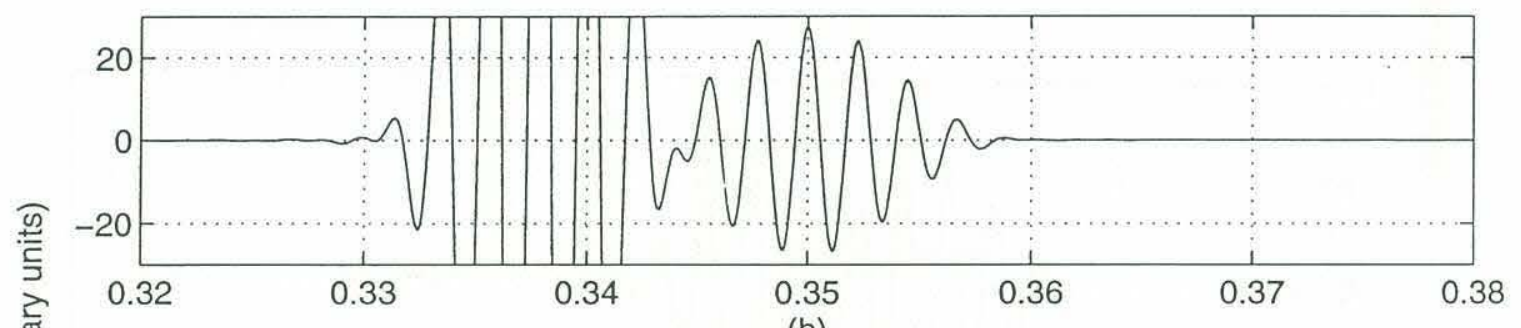

(b)

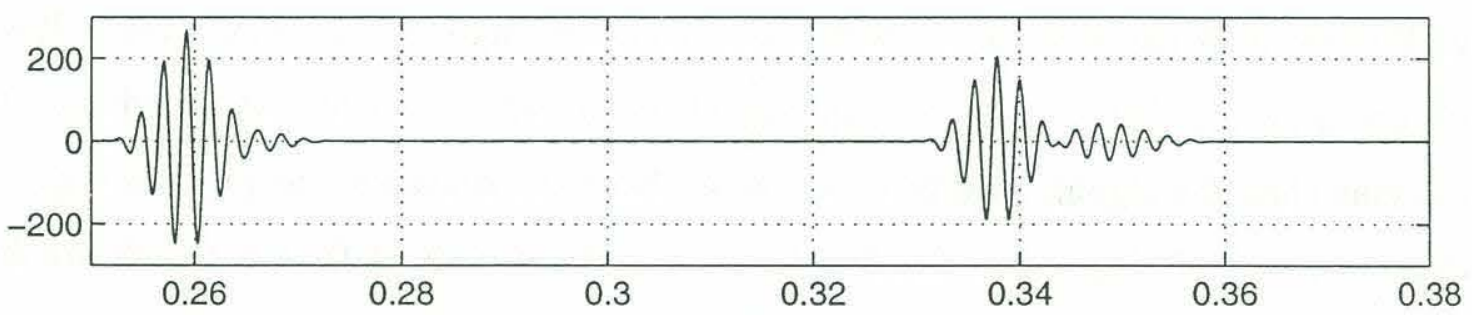

(d)

Time (sec)

Figure 2-13: (a) The returned signal from the target alone; (b) The signals from the scatterers in the second layer; (c) Part of (d) the entire received signals at the bottom hydrophone. 


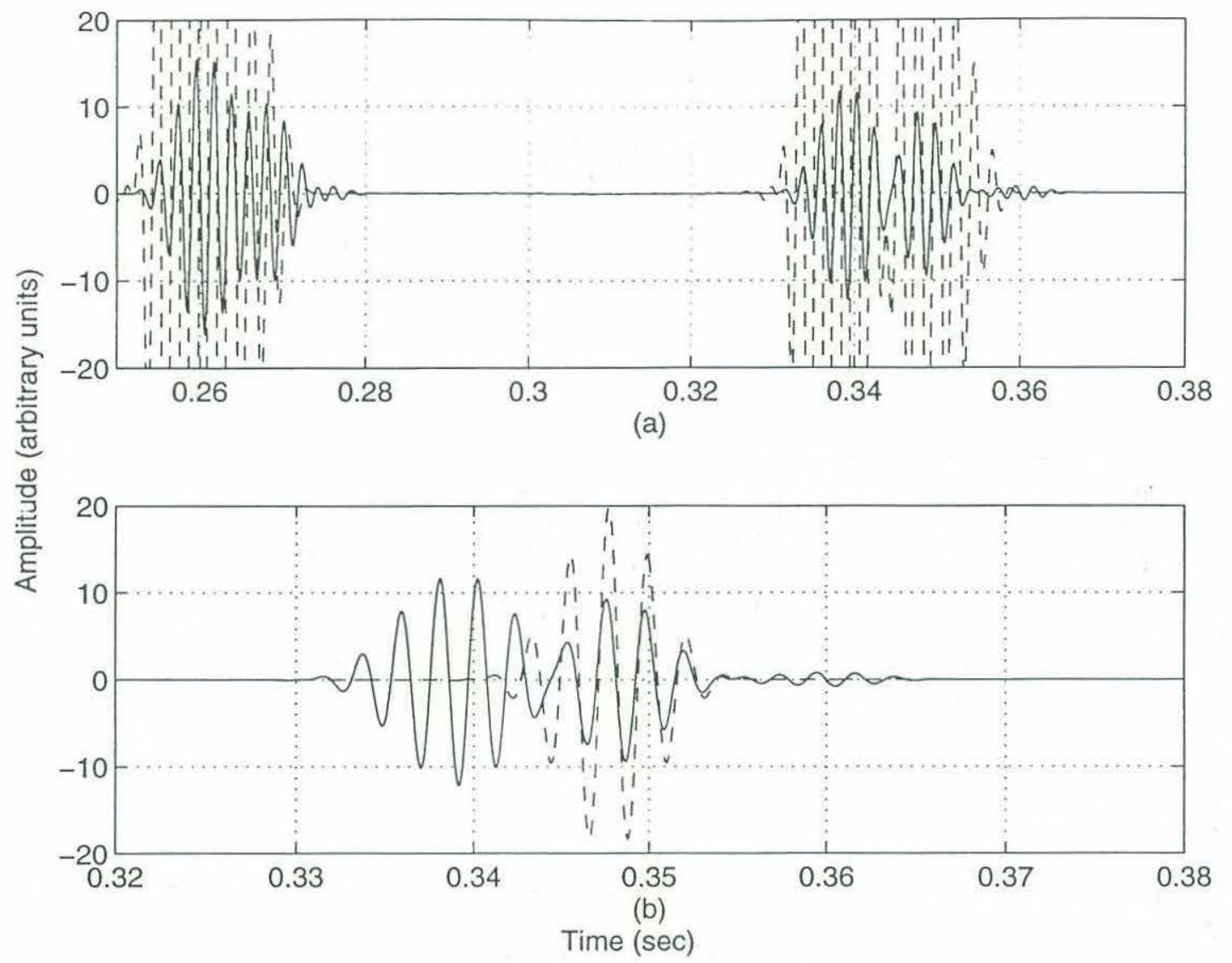

Figure 2-14: The single-constraint beamforming results: (a) the received signals at the bottom hydrophone (dashed line) and the beamformer output (solid line); (b) the target signal (dashed line) and the beamformer output (solid line).

Figure 2.14 are the results of the single-constraint beamformer. The solid lines in Figures 2.14 (a) and (b) represent the beamforming output. The dashed line in Figure 2.14 (a) denotes the original signals collected at the bottom receiver. It is obvious that the signals at $0.26 \mathrm{~s}$, which are from the scatterers at grazing angles 90 degrees and 75.5 degrees in the first layer, and the signals at $0.34 \mathrm{~s}$, which are from the scatterer at grazing angle 90 degrees in the second layer, have been attenuated to some degree. However, in Figure 2.14 (b), only the part of signals close to the target arrival time is shown. Here the dashed line represents the signal from the target alone. It can be seen that the target signal has not been satisfactorily recovered and the unwanted signal level is still higher than that of the target. So the single 


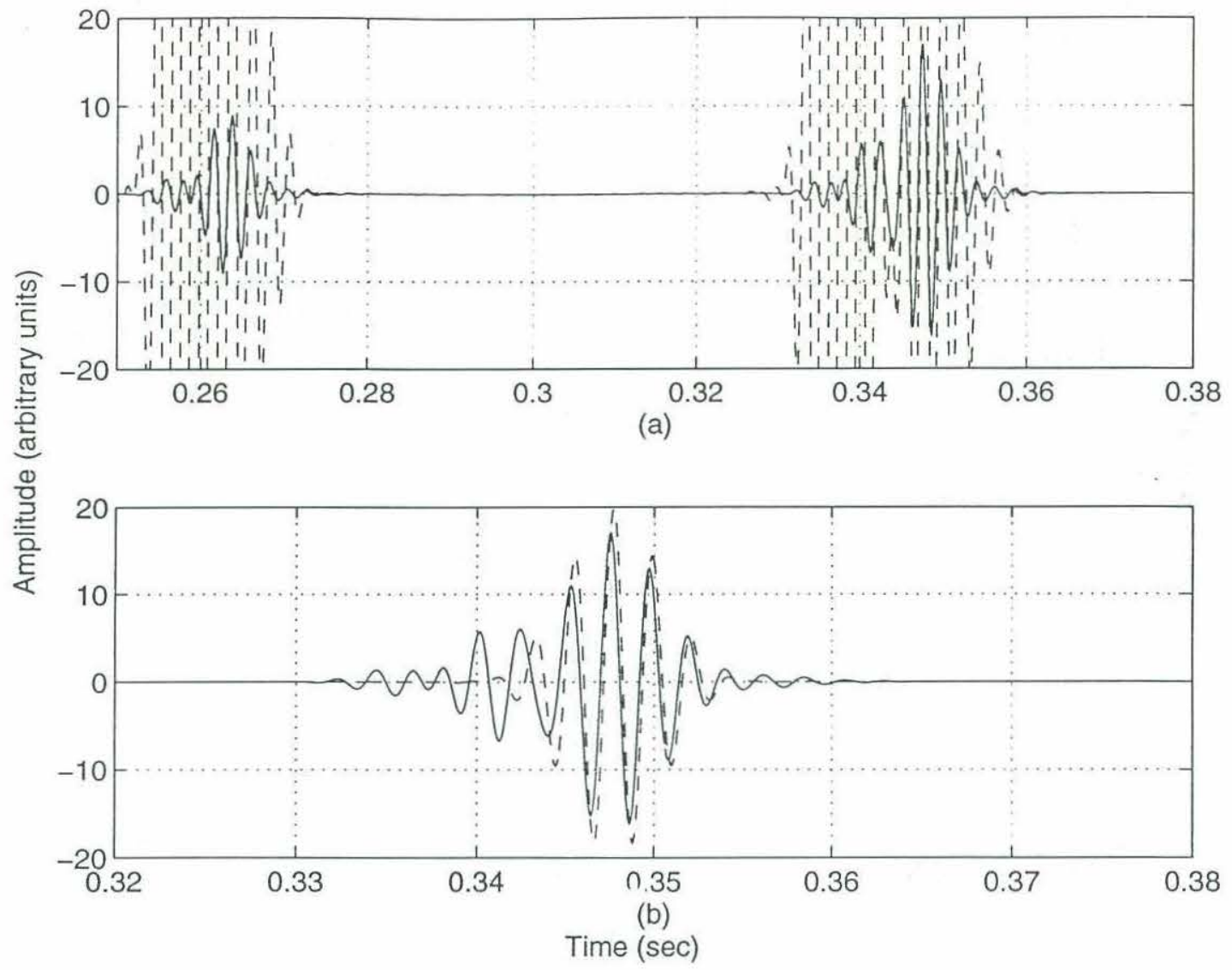

Figure 2-15: The multiple-constraint beamforming results: (a) the received signals at the bottom hydrophone (dashed line) and the beamformer output (solid line); (b) the target signal (dashed line) and the beamformer output (solid line).

constraint beamformer is not good enough in discriminating the target signal against the interference from other directions.

The multiple constraints beamformer simulation results are shown in Figure 2.15. Again, the solid lines in Figure 2.15 (a) and (b) represent the beamformer output. The dashed line in Figure 2.15 (a) represents the signals collected at the bottom receiver. Compared with the results in Figure 2.14(a), it can be seen that the amplitude of residual signals from other than desired direction are smaller, which means higher attenuation and better rejection using the multiple constraints beamformer. Also notice in Figure 2.15 (b), the beamformer output is close to the pure target signal, which is denoted by a dashed line. This suggests that by using the multi- 


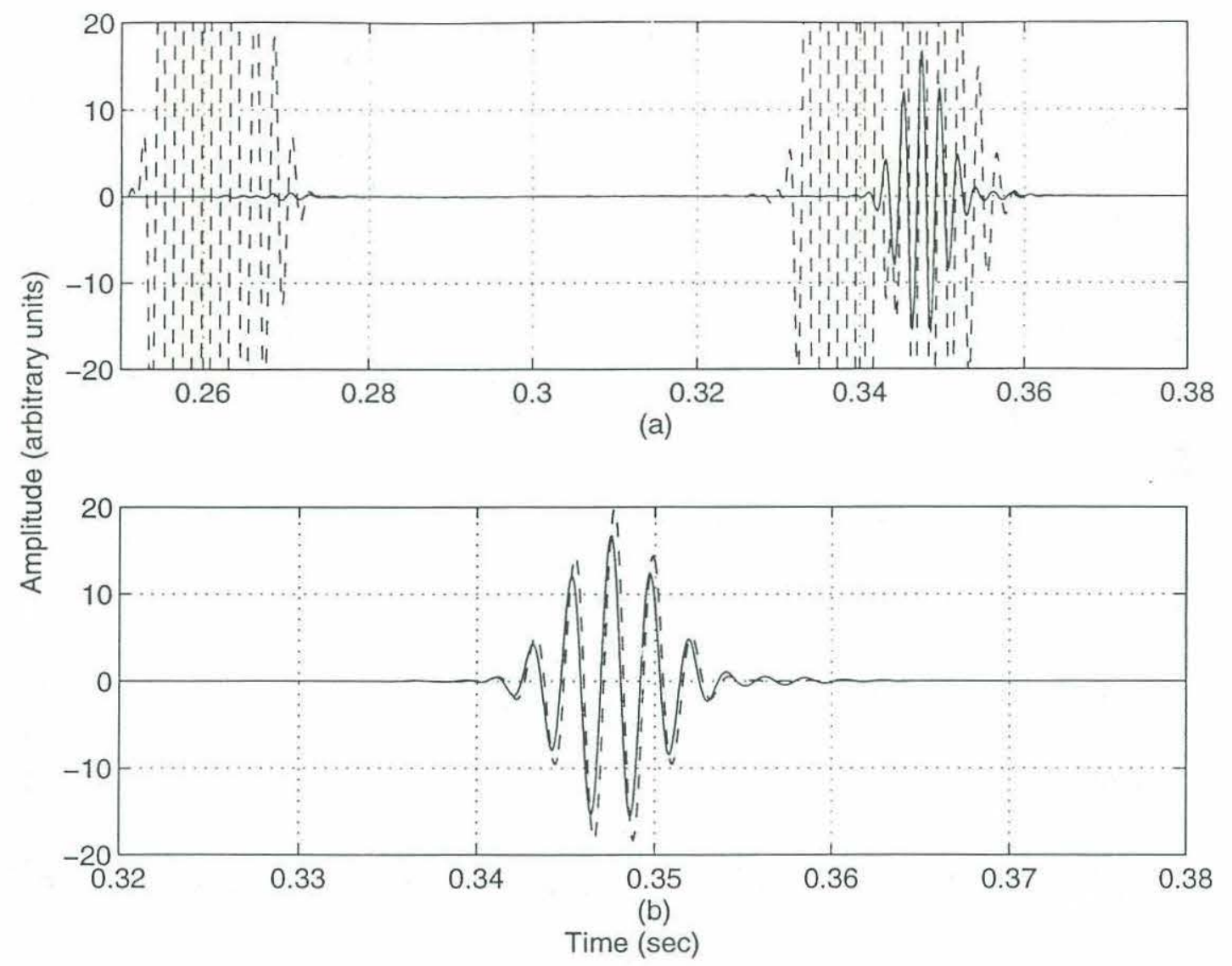

Figure 2-16: The hybrid method results: (a) the received signals at the bottom hydrophone (dashed line) and the hybrid method output (solid line); (b) the target signal (dashed line) and the hybrid method output (solid line).

ple constraints beamforming technique, the signals from a desired direction can be successfully extracted in the presence of strong directional interferences. This will benefit the backscattering data analysis to be presented in Chapter 3 .

Another approach is a hybrid of multiple constraint beamforming and subtraction of the normal and near-normal incidence returns. This method is designed especially for the ARSRP experiment, and it will be described in detail in Chapter 3. The idea is to obtain the normal and near normal incidence returns by steering the beam in the normal incidence direction using the single-constraint beamformer, subtracting this part of the signal from the total signal, and then applying the multiple constraints beamforming to the remaining part. Figure 2.16 shows the results of this method. 
The solid lines in Figures 2.16 (a) and (b) represent the beamformer output. The dashed line in Figure 2.16 (a) denotes the collected signal at the bottom receiver. The dashed line in Figure 2.16 (b) denotes the target signal. It can be seen the signals from unwanted directions are almost totally rejected. The beamforming output matches satisfactorily the target signal, as shown in Figure 2.16 (b).

\subsection{Array Element Localization}

With the development of technology, an acoustic array is capable of being towed underwater up to $6000 \mathrm{~m}$ beneath the sea surface. In order to take full advantage of available signal processing techniques, such as beamforming and matched field processing, the locations of all the hydrophones on a towed array, or at least the array shape, must be known with respect to a reference coordinate system.

Generally, Array Element Localization (AEL) sensors are attached to an array and used to determine the depth of the array elements below the sea surface, the array heading and the straightness of the array [45] [46]. However, in some of the experiments, these sensors are not available or are sometimes malfunctioning. Therefore, it is of great importance to have some alternative methods to localize the array elements.

In the scattering experiment that we will discuss in the next chapter, the acoustic source is towed only $200 \mathrm{~m}$ to $400 \mathrm{~m}$ above the seafloor, with a 24 hydrophone receiving array hung beneath the source. For a source/receiver geometry like this, we can exploit the information contained in the direct arrival and the bottom reflected arrival from the source to each receiver. The position of the source and positions of each of the receivers relative to the source can be obtained using the following estimation algorithm.

Figure 2.17 shows the geometry of the acoustic source/receiver system. Here 


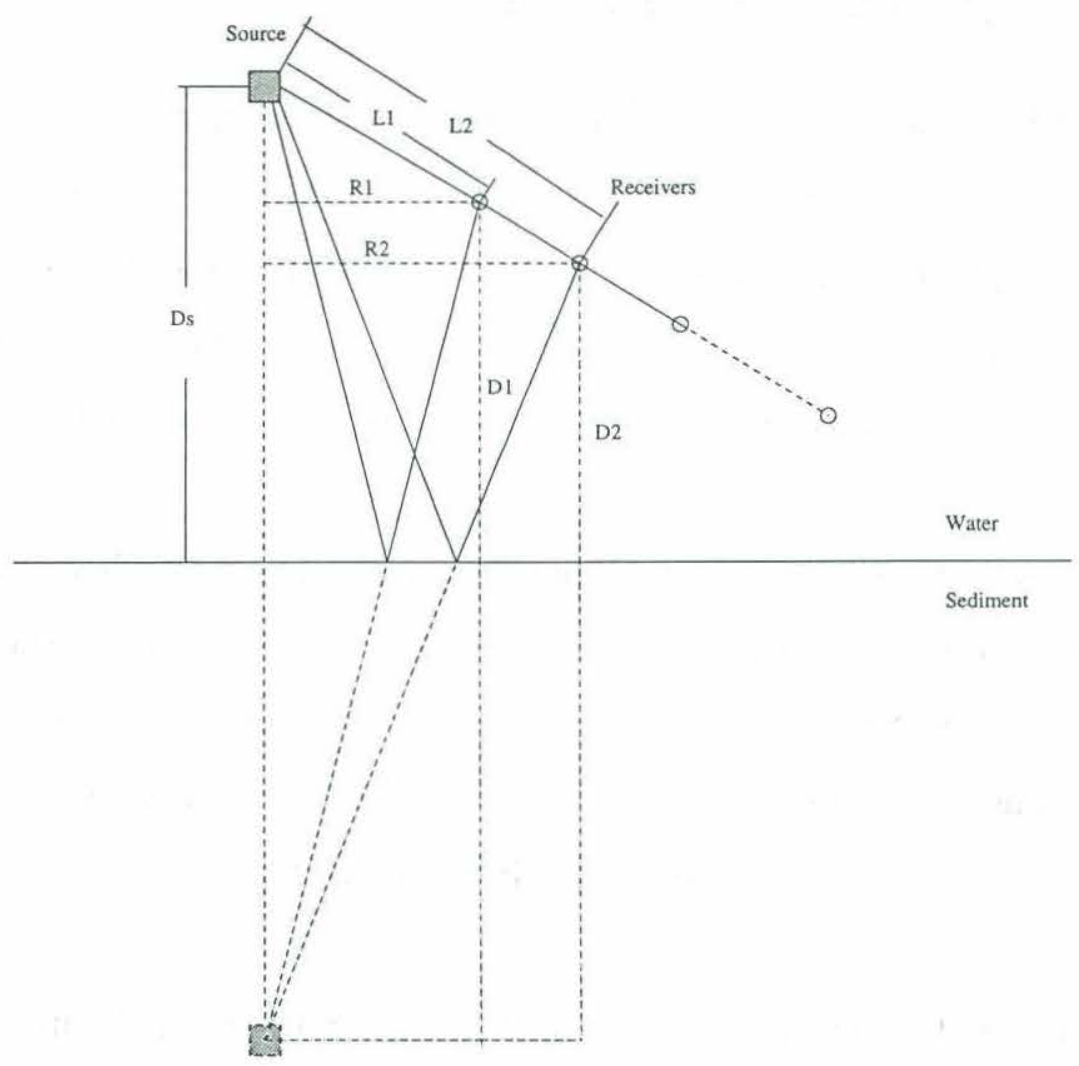

Figure 2-17: Scheme of the array element localization algorithm. 
$D s$ represents the height of the source above the seafloor and $D_{i}(i=1,2, \ldots, N)$ represent the heights of the receiving elements above the seafloor, where $\mathrm{N}$ is the number of receiving array elements. $R_{i}(i=1,2, \ldots, N)$ are the horizontal ranges between the source and the receivers. $L_{i}(i=1,2, \ldots, N)$ are the distances between the source and the receivers. Let $T D_{i}(i=1,2, \ldots, N)$ stand for the direct arrival times from the source to the receivers and $T R_{i}(i=1,2, \ldots, N)$ be the bottom reflected arrival times from the source to the receivers. Since the sound speed gradient is small, over a short vertical distance, we assume that the sound velocity is a constant $c$ between the source and the seafloor, with $c$ being the average of the measured sound speeds at the receiver and near the bottom. We have:

$$
\begin{aligned}
L_{i} & =c \cdot T D_{i}, \\
R_{i} & =\sqrt{\left(c \cdot T R_{i}\right)^{2}-\left(D s+D_{i}\right)^{2}}, \\
R_{i} & =\sqrt{L_{i}^{2}-\left(D s-D_{i}\right)^{2}}, \quad i=1,2, \ldots, N .
\end{aligned}
$$

By plugging Eq. 2.19 into Eq. 2.21 and then subtracting Eq. 2.21 from Eq. 2.20, we obtain the following equations after some rearrangements:

$$
\begin{aligned}
D_{i} & =\frac{c^{2} \cdot\left(T R_{i}^{2}-T D_{i}^{2}\right)}{4 D s}, \\
R_{i} & =\sqrt{\left(c \cdot T D_{i}\right)^{2}-\left(D s-D_{i}\right)^{2}}, \quad i=1,2, \ldots, N .
\end{aligned}
$$

Since $T D_{i}$ and $T R_{i}$ can be obtained from the measurements, the variable $D s$ will determine $D_{i}$ and $R_{i}(i=1,2, \ldots, N)$ uniquely. The remaining question is how to obtain $D s$.

If we consider two neighboring hydrophones and the source, there are five unknown parameters, i.e., $D s, R_{j}, D_{j}, R_{j+1}$, and $D_{j+1}, j=1,2, \ldots, N-1$. However, there are only four constraints, which are the direct and bottom reflected arrival times, $T D_{j}, T R_{j}, T D_{j+1}, T R_{j+1}$. In order to obtain a unique solution to this problem, another constraint must be included. Here we define the distance between two 
neighboring hydrophones to be:

$$
\delta L_{j}=\sqrt{\left(D_{j}-D_{j+1}\right)^{2}+\left(R_{j}-R_{j+1}\right)^{2}}, \quad j=1,2, \ldots, N-1 .
$$

Usually in the experiment, the hydrophones are connected to each other by cables. The cable lengths between the hydrophones are measured before the deployment. We will only consider equally spaced arrays and use $h s$ to represent the cable length between two neighboring hydrophones. When $h s$ is not too large, we can make the assumption that $\delta L_{j}$ is as close to $h s$ as possible. This acts as an additional constraint that we need to determine the array element locations. As a result, the source and receiver positions can be obtained for each pair of hydrophones. However, the approach using only two hydrophones is very sensitive to the error associated with arrival time estimation, and a unique solution for the entire array cannot be achieved. Therefore, we define a cost function as follows:

$$
Q=\sum_{j=1}^{N-1}\left(\delta L_{j}-h s\right)^{2}
$$

We can search all possible $D s$ 's to minimize the cost $Q$. The $D s$ corresponding to the minimal $Q$ is considered the desired value. This process can be expressed as:

$$
D s=\min _{D s} Q
$$

This method has been tested using real data and the results have been compared with those obtained from the array element localization sensors. Figure 2.18 shows the results of a typical test using the experimental data collected by a deep-towed horizontal line array in the scattering experiment described in the beginning of this chapter. The cable length between adjacent hydrophones is $2.1 \mathrm{~m}$. The *'s represent the hydrophone positions inferred from the Array Element Localization sensors. The o's represent the results obtained from the above-mentioned estimation algorithm. It can be seen that they have very good agreement. 


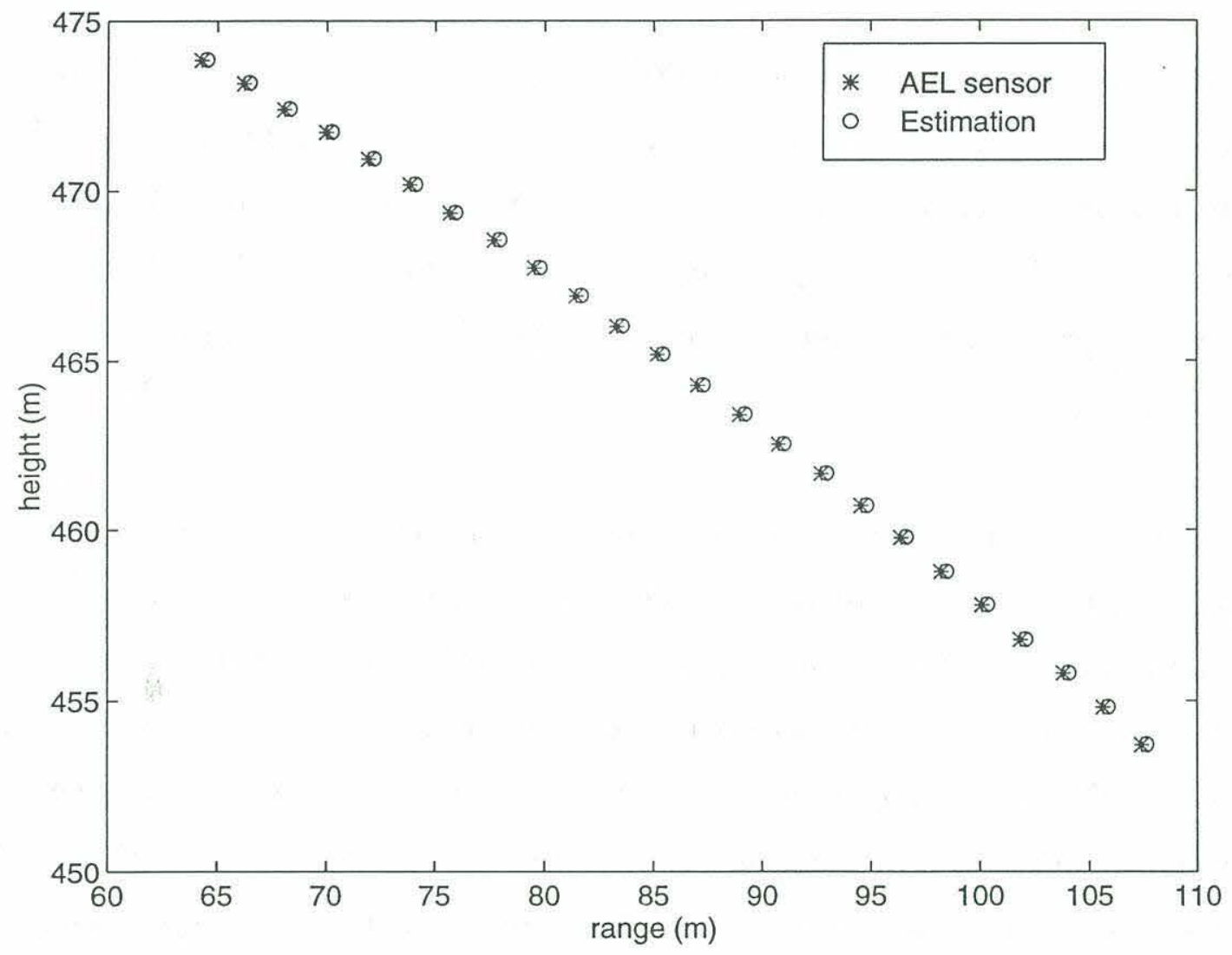

Figure 2-18: Hydrophone positions: the horizontal range from the source and the height above the seafloor. 


\subsection{Bottom Backscattering Strength}

The backscattering strength (BSS) is employed to characterize the bottom backscattering process quantitatively, with the a priori assumption that the scattering process takes place at the water-bottom interface. It is defined as follows:

$$
B S S=10 \log _{10} \frac{I^{s} r^{2}}{\left(I^{\text {in }} \cdot A\right)},
$$

where $I^{s}$ is the intensity of backscattered signals at the receiver, $I^{\text {in }}$ is the intensity of the incident sound wave a unit distance away from the scattering area, $r$ is the distance from the source to the scatterer, and $A$ is the insonified area [47]. Note that the backscattering strength depends on the grazing angle of the incident wave relative to the scattering surface, which is not necessarily the water-bottom interface. This idea will be pursued further in Chapter 3.

In the single source/single receiver geometry and when the source and receiver are close to each other, the following formula is deduced to calculate the backscattering strength from the collected data. As shown in figure 2.19, the annulus with width $\Delta x$ is the insonified area $A$. The horizontal range between the source and the scattering area is $x$. The grazing angle with respect to the scattering surface is $\theta$. The height of the source above the scattering surface $H$ is assumed much larger than the distance between the source and the receiver $d$. Let $c$ represent the sound speed and $t$ the round trip travel time. $\tau$ is a constant, which is the pulse length for the source. From the source/receiver geometry, we have:

$$
\begin{aligned}
r & =\frac{c t}{2} \\
A & =2 \pi x \Delta x \\
& =2 \pi \frac{H}{\tan \theta} \cdot \frac{c \tau}{2 \cos \theta} \\
& =\frac{2 \pi H c \tau}{2 \sin \theta} \\
& =\frac{\pi c^{2} \tau t}{2}
\end{aligned}
$$




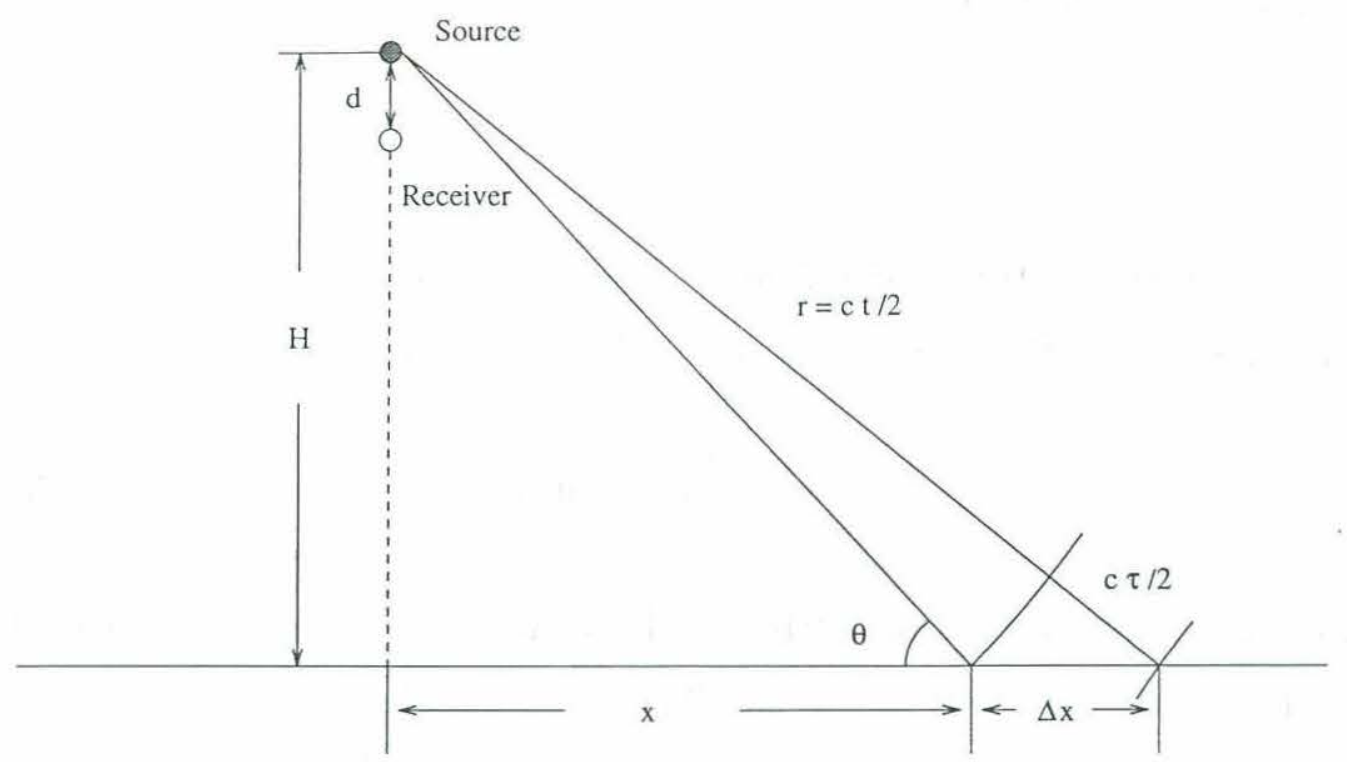

Figure 2-19: The conventional single source/single receiver configuration.

$$
=\pi c \tau r \text {. }
$$

Let $I^{d}$ be the intensity of the incident wave at the receiver. Then

$$
I^{i n} \cdot r^{2}=I^{d} \cdot d^{2},
$$

or

$$
I^{\text {in }}=I^{d} \frac{d^{2}}{r^{2}} .
$$

Equation 2.27 now becomes:

$$
\begin{aligned}
B S S & =10 \log _{10} \frac{I^{s} r^{2}}{\left(I^{i n} \cdot A\right)} \\
& =10 \log _{10} \frac{I^{s} r^{3}}{I^{d} d^{2} \pi c \tau} \\
& =10 \log _{10} \frac{I^{s}}{I^{d}}\left(\frac{c t}{2}\right)^{3} \frac{1}{d^{2} \pi c \tau},
\end{aligned}
$$


and $I^{d}$ can be obtained from:

$$
I^{d}=\frac{1}{\tau} \int_{t_{0}}^{t_{0}+\tau} P^{2}(t) d t,
$$

where $t_{0}$ is the one way travel time from the source to the receiver and $P(t)$ is the time series recorded at the receiver. Similarly, $I^{s}$ can be calculated:

$$
I^{s}=\frac{1}{\tau} \int_{t}^{t+\tau} P^{2}(T) d T .
$$

As mentioned earlier, $t$ is the round trip travel time from the source to the scatterers and from the scatterers to the receivers. The backscattering strength can be estimated from the received signals using the above formulas. If attenuation is taken into consideration, the amount $2 \alpha r$ should be added to the above backscattering strength BSS, where $\alpha$ is the attenuation coefficient.

Directional receiving/transmitting arrays are another configuration often used to measure the backscattering strength. This enables the application of signal processing techniques such as beamforming to resolve the scattered returns from different directions. However, the directivity aspect makes it difficult to determine the insonified area precisely. Also, the absolute calibration of the array becomes necessary. Here, we just consider the simple case where the return is from the maximum response axis (MRA) of the array, i.e., the returned signals suffer no attenuation passing through the beamformer. The insonified area $A$ is now calculated as the smaller value of the following two quantities [48]:

3- $d B$ area:

$$
A_{\theta_{3 d B}}=2 \pi \theta_{3 d B} \frac{r^{2}}{\sin \theta} \cos \theta,
$$

$c \tau / 2$ area:

$$
A_{\tau}=\pi c \tau r,
$$

where $\theta_{3 d B}$ is the $3 d B$ beamwidth (in radians), and $\theta$ is the grazing angle of the main lobe axis. The above-mentioned $3 d B$ area is the annulus where the array response is 
within $3 d B$ compared to the MRA. The $c \tau / 2$ area is the one pulse-length insonified ring, as in the single source/single receiver geometry. $I^{\text {in }}$ can be computed using Eq. 2.31 and Eq. 2.33. $I^{s}$ can be obtained using Eq. 2.34. Instead of being the time series at one hydrophone, $P(t)$ is the beamformed output from all the hydrophones, where $t$ is determined from the distance between the source/receiver and the scatterers at a particular grazing angle. This method will be used in Chapter 3 to calculate the backscattering strengths of two inhomogeneous scattering layers from the data collected by a vertical line array. 


\section{Chapter 3}

\section{Experimental Data Analysis}

\section{Results}

\subsection{Preprocessing}

A procedure called preprocessing is often applied to experimental data. The purpose of this process is to improve the data quality and satisfy the requirements of further processing.

Due to the existence of ambient noise in the ocean, the recorded signals are often contaminated. As shown in Figure 2.4, the noise level is about $-25 \mathrm{~dB}$ relative to the direct arrival signal level. However, it is still comparable to the scattered signals at low grazing angles. Therefore, the data is passed through a bandpass filter first, which has a passband of $250-650 \mathrm{~Hz}$, to alleviate the noise effects. Figure 3.1 shows the filtered output at one hydrophone and its spectrum. The noise outside the passband is greatly attenuated, which is important for the analysis of the backscattered signals at low grazing angles.

The next step is upsampling. We want to obtain the normal incidence direction returns from the bottom using time domain beamforming in the subsequent processing. This procedure requires that the sampling frequency be at least 5-10 times higher 

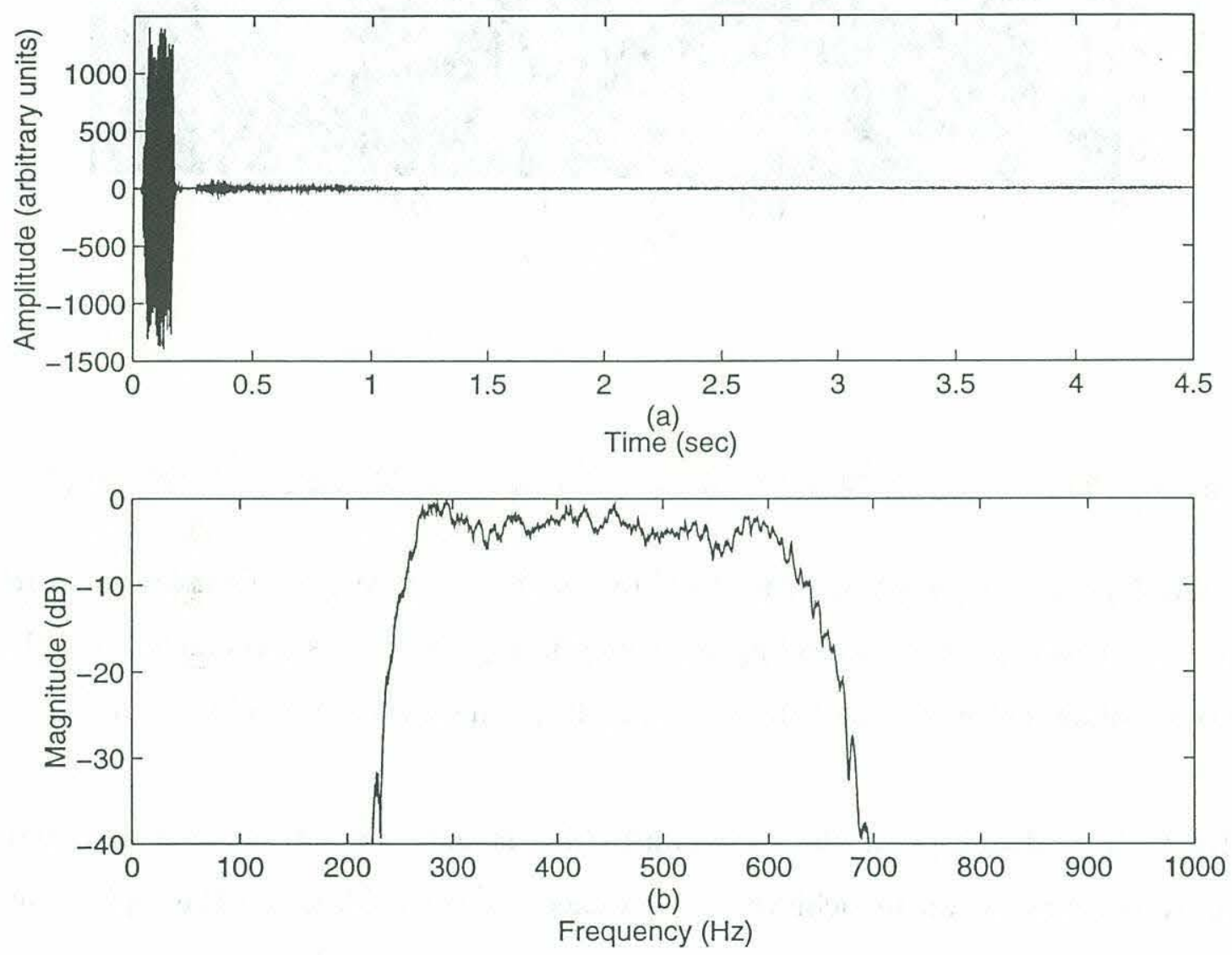

Figure 3-1: (a) The bandpass filter output and (b) its spectrum for the signals at one hydrophone for a typical ping. 


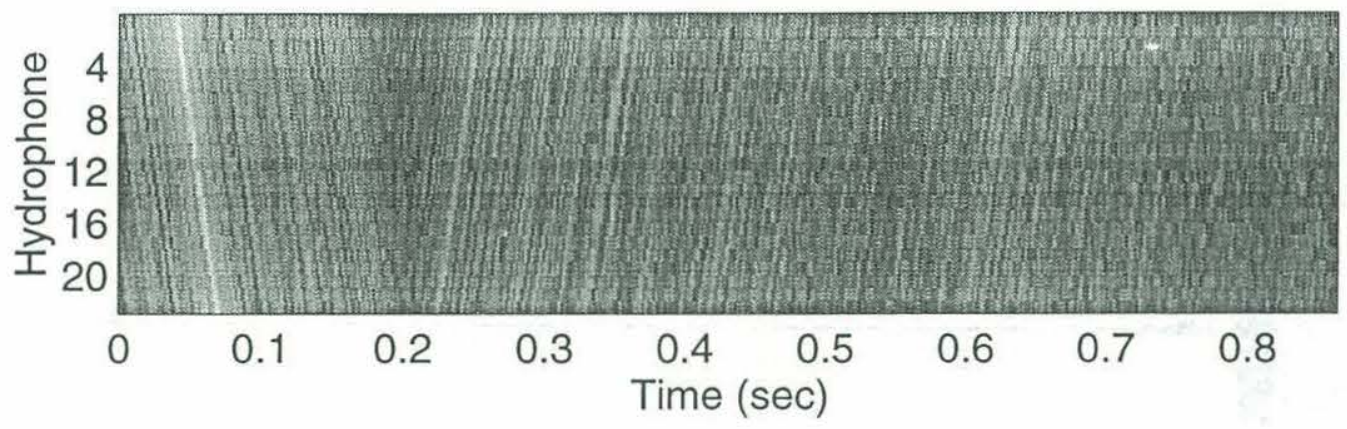

Figure 3-2: The signals at 24 hydrophones after matched filtering for a typical ping than the Nyquist frequency in order to obtain satisfactory results. Considering during the data collection the sampling frequency is kept as low as possible due to the limitation of data storage, the data at each hydrophone is upsampled by 8 times here.

By taking advantage of the wide-band chirp signals, high resolution and high signal-to-noise ratio can be achieved by matched filtering. Choosing the replica signal to be the direct arrival recorded on the first hydrophone, the noise uncorrelated with the source signal is very much suppressed and the signal-to-noise ratio is improved. This makes it easier to identify some intrinsic scattering features. Figure 3.2 depicts the signals at all hydrophones after the processing. The brighter part has larger amplitude. The strong returns from the sediment layering (white lines) can be seen clearly at later time (greater than $0.2 \mathrm{~s}$ ), which have the opposite slope to that of the direct arrival at the beginning (around $0.08 \mathrm{~s}$ ). 
In the next section, the beamforming technique will be used to extract the scattered returns in normal incidence and oblique directions from the preprocessed data.

\subsection{Analysis of Signals in the Normal Incidence Direction}

Primary goals of this experiment are to identify the dominant scatterers' locations and to estimate their scattering strengths. However, this is unlikely to be accomplished without knowledge of the sediment structure.

At high frequencies, significant sediment attenuation limits the penetration of acoustic energy into the sediment and only the very top layer can be insonified. Therefore, the conventional assumption that all the scatterers are located at the water-sediment interface is acceptable. However, at low frequencies, the situation is generally different. As Merklinger [32] pointed out, not only surface roughness but also sub-bottom inhomogeneities can play important roles in the backscattering process. Even at relatively high frequencies, Lyons et al. [23] recently found that the inhomogeneous continuum and the internal rough interfaces in the sediment were important contributors to the backscattering. However, pinpointing these scatterers' locations is an arduous task. It would be ideal to have the ground truth core data at the experimental site, but typically these data are unavailable. In the ARSRP experiment, core data exist for 5 stations across the sediment pond. Taking into account the fact that the core depth is 5 meters, only limited information is provided. For sediment with thickness up to $400 \mathrm{~m}$, it is therefore necessary to acquire knowledge of the sediment structure from acoustic data analysis.

Work in the seismic community has shown that seismic signals in the normal incidence direction provide useful information about the geological structure of the 
ocean bottom. A cross-section of the survey area can be obtained from the normal incidence direction returns collected on the receiving array [49]. In our experiment, the vertical line array was towed across the entire sediment pond, and the normal incidence return was extracted at each ping location.

The signal recorded on each hydrophone is the sum of returns from all directions. It includes the backscattering from the water-sediment interface, from the volume inhomogeneities within the sediment, and from the basement, as well as the coherent reflections from the water-bottom interface and sediment internal layers. With a single omnidirectional receiver, one cannot discriminate signals in the normal incidence direction from those in other directions. The beamforming technique introduced in Chapter 2 will be used to obtain signals in the desired direction and attenuate returns in all other directions.

Assume the beam of the array is steered in the normal incidence (endfire) direction. Since the array is in a vertical configuration, endfire beamforming in the frequency domain is equivalent to the delay-and-sum method in the time domain. We align the time series with an appropriate delay for each hydrophone according to the reflected arrival from the water-sediment interface and then sum them up. Compared with the frequency-domain approach, it is computationally more efficient to use the delay-and-sum method in obtaining the normal incidence direction returns. Upsampling of the data will enable better alignment of the arrivals and better operation of the time-domain beamformer.

Figure 3.3 depicts the beampattern for endfire beamforming at the center frequency $450 \mathrm{~Hz}$, where the geometry of the vertical line array in the experiment is used. The $-3 \mathrm{~dB}$ beamwidth is about 30 degrees, which means that the beamforming output will include returns from a 30 degree cone. Since the source signal is in the frequency range $250-650 \mathrm{~Hz}$ and the beampattern changes with frequency, the actual beampattern will be the weighted average of the beampatterns at all the frequen- 


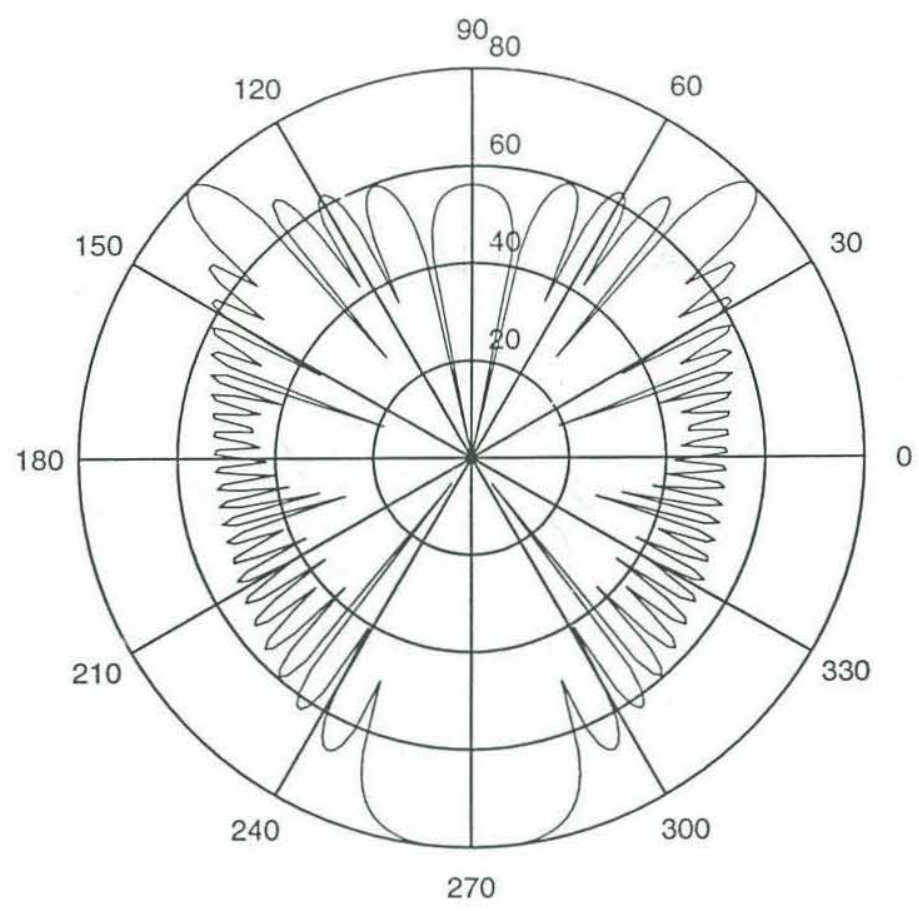

Figure 3-3: Beampattern for the endfire beamformer at $450 \mathrm{~Hz}$

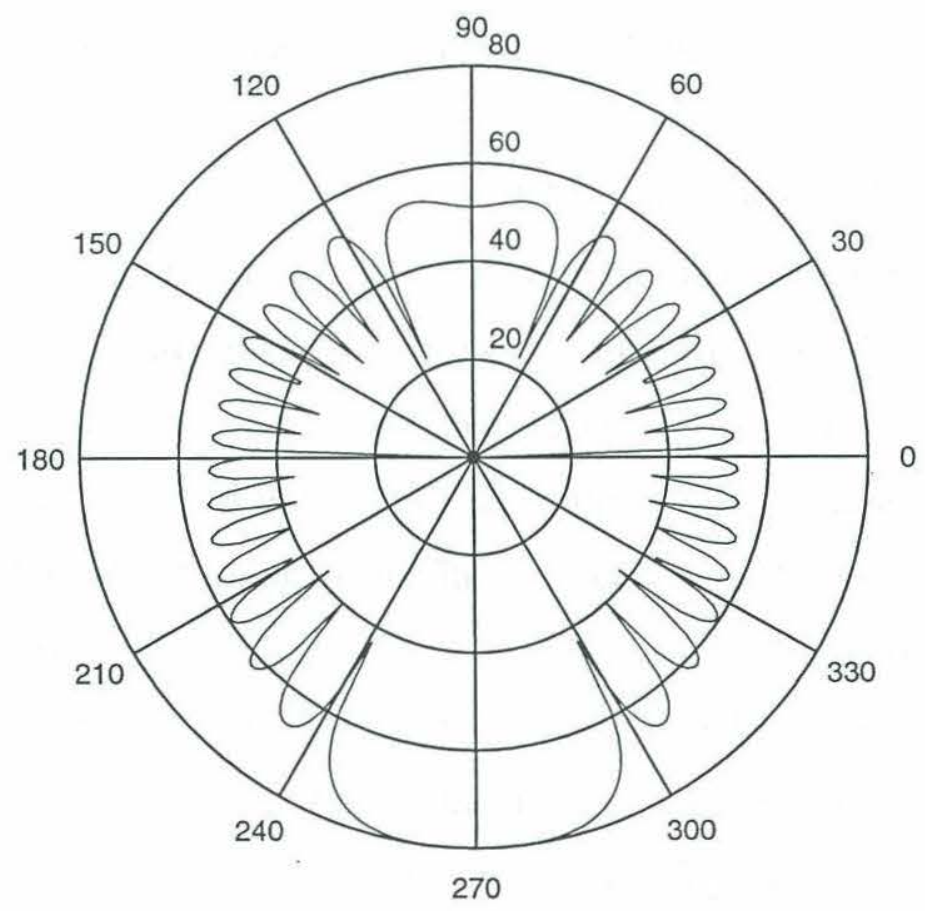

Figure 3-4: Beampattern for the endfire beanformer at $250 \mathrm{~Hz}$ 


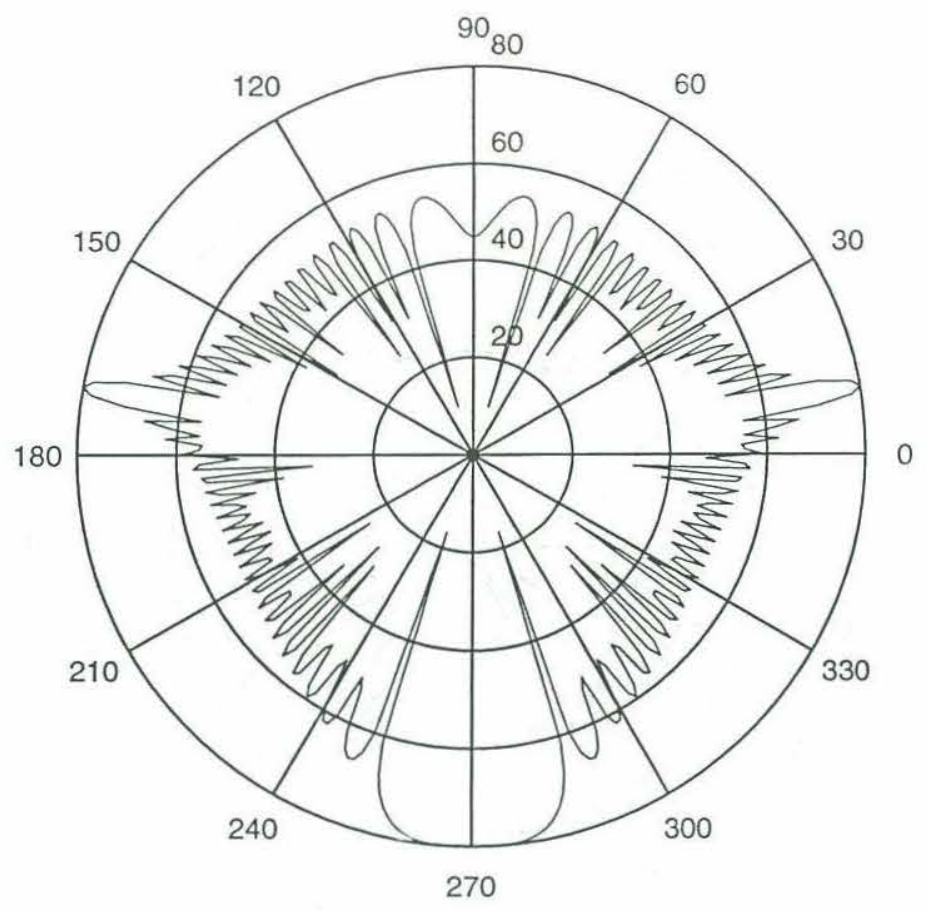

Figure 3-5: Beampattern for the endfire beamformer at $650 \mathrm{~Hz}$.

cies. Figure 3.4 and 3.5 show the beampattern at frequencies $250 \mathrm{~Hz}$ and $650 \mathrm{~Hz}$ respectively. Notice that the beam is wider for lower frequencies. Nonetheless, the beampattern at the center frequency still provides a sufficient estimation.

After applying the aforementioned time domain beamforming technique to the preprocessed data, we obtained the normal incidence direction returns for each ping. Figure 3.6(a) shows the recorded signal after preprocessing on the bottom hydrophone for a typical ping. Here time 0 corresponds to the trigger of transmission. Figure 3.6(b) shows the beamformed output with the normal incidence direction being the look direction. Figure 3.6(c) shows the difference signal between these two, which consists primarily of backscattered signals coming from other than the normal incidence direction, together with some small normal incidence direction contributions due to sidelobe leakage in the endfire beamformer. From the plot, it can be seen that the curves in Figure 3.6(a) and (b) correlate very well except after $0.65 \mathrm{~s}$, when the basement returns arrive. The amplitude of the difference signal is much smaller compared with that of the beamformed output. Since the endfire beamforming re- 


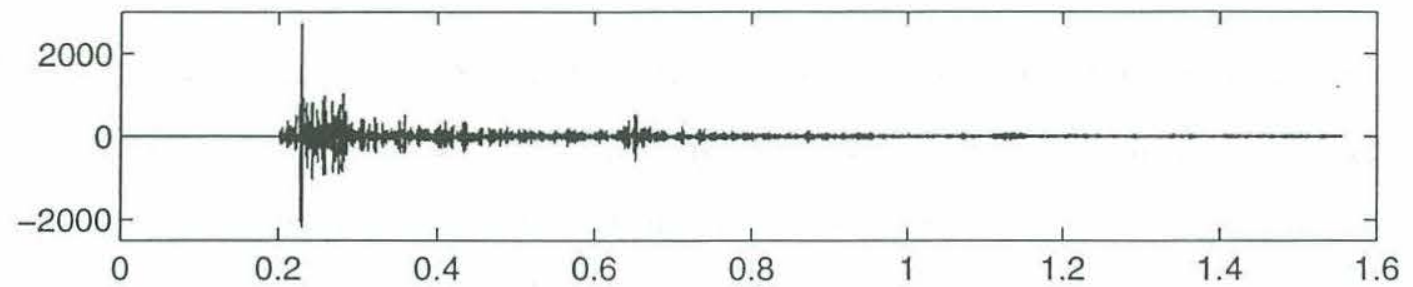

(a)
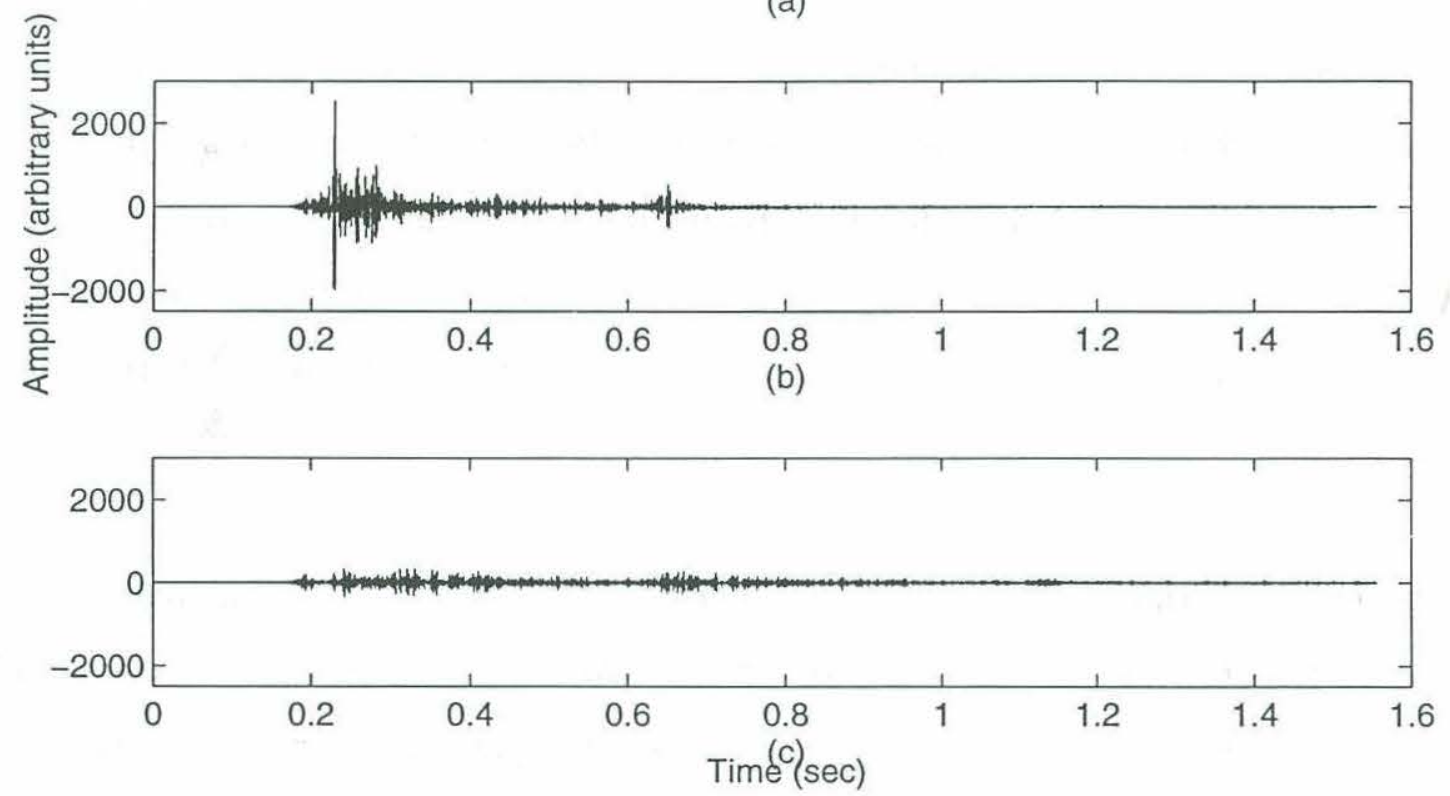

Figure 3-6: For a typical ping: (a) the signal on the bottom hydrophone after matched filtering, (b) the return in the normal direction obtained by endfire beamforming, (c) the difference signal between (a) and (b). 
sults contain returns in the normal and near-normal incidence directions only and signals at single hydrophone are the bottom reverberation in all directions, the above good correlation can be interpreted as showing that bottom returns in the normal and near-normal incidence directions domincte the total bottom reverberation. Considering that normal incidence returns consist primarily coherent reflections of the acoustic energy at layer interfaces, a layered structure of the sediment is suggested.

However, it is not appropriate to identify the layered structure, which usually spreads over a large area, by the phenomenon that is observed at one position, i.e., from single ping data. Therefore, the above endfire beamforming technique is applied to a large number of consecutive pings. With the ship moving across the sediment pond, the sediment profiling can be obtained by aligning the normal incidence direction returns according to the water-sediment interface reflected arrivals. From now on, the time 0 will be chosen to be the arrival time of the first water-sediment interface reflection. Figure 3.7 shows the cross-section of part of the sediment pond. It can be seen that layering with gentle horizontal changes is the main feature of the sediment.

The lateral variations of sediment properties usually play an important role in bottom backscattering. With the DTAGS being towed across the sediment pond and a ping to ping distance of $7.8 \mathrm{~m}$, we are able to investigate the horizontal variations by analyzing the endfire beamforming data for multiple pings. In Figure 3.8(a), the normal incidence direction returns for 8 consecutive pings collected at the east side of the sediment pond have been plotted over each other (Here only the first $0.12 \mathrm{~s}$ data are plotted). It shows that the 8 pings are well correlated between $0 \mathrm{~s}$ and 0.02 $\mathrm{s}$ and between $0.045 \mathrm{~s}$ and $0.065 \mathrm{~s}$, while the correlation in other parts of the signal is poor. The averaged normal incidence direction return of the 8 pings is shown in Figure 3.8(b), i.e.,

$$
\bar{B}(90, t)=\frac{1}{N} \sum_{i=1}^{N} B\left(90, t, x_{i}\right), \quad(N=8) .
$$




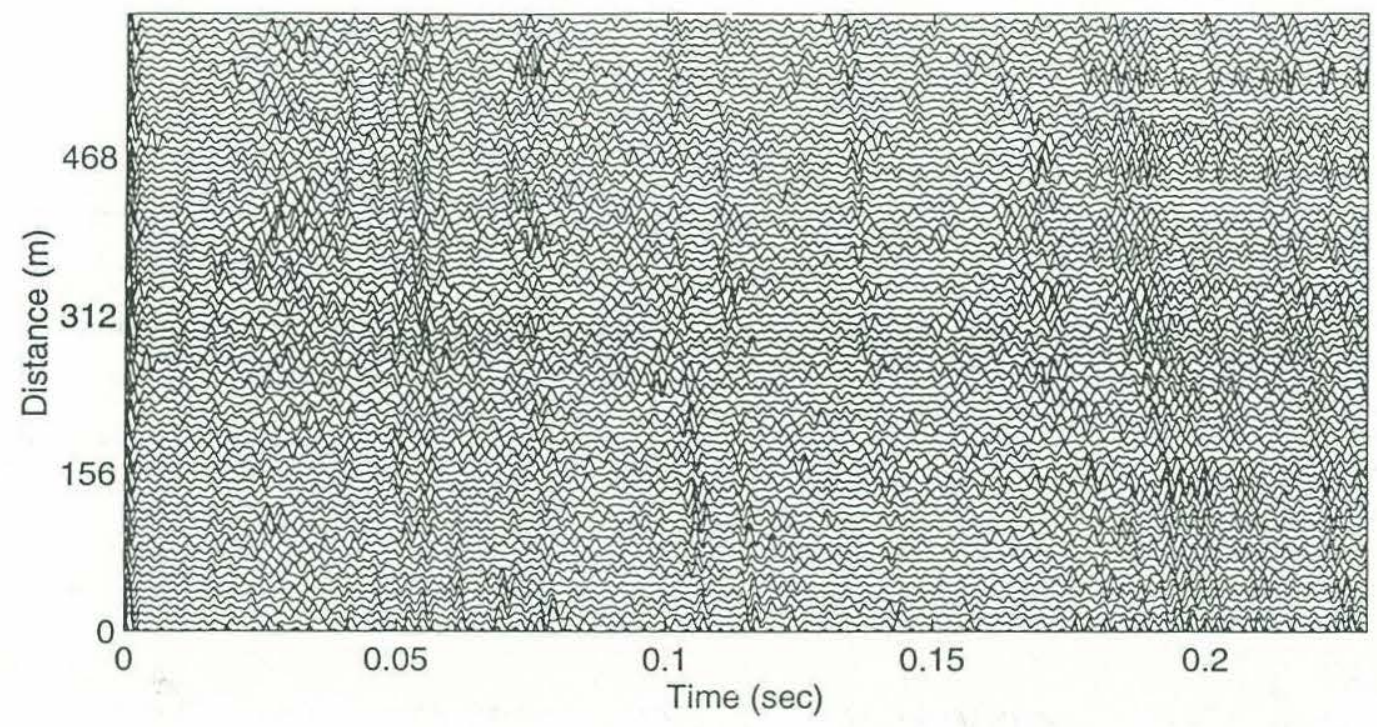

Figure 3-7: The cross-section of part of the sediment pond.

where $B\left(90, t, x_{i}\right)$ is the beamformed output at grazing angle 90 degrees (normal incidence direction) for the $i$ th ping, $\bar{B}(90, t)$ is the average, and $N$ is the number of pings being averaged. Notice that curves for the two poorly correlated regions are relatively flat and have low amplitudes. Figure 3.8(c) shows the difference signals,

$$
D\left(90, t, x_{i}\right)=B\left(90, t, x_{i}\right)-\bar{B}(90, t), \quad i=1,2,3, \ldots 8 .
$$

which can be considered mainly the incoherent scattering in the normal incidence direction. In this plot, the poorly correlated regions display larger amplitudes. As mentioned earlier, normal incidence returns correspond primarily to coherent reflections from the layer interfaces. The strong correlation of returns among many pings in an area indicates that horizontal layering is the underlying feature. For the poorly correlated parts, random regions instead of well defined layers are more likely to be the corresponding features. So two random regions are regarded to be present in this part of the sediment pond. For the west side of the sediment pond, Figures 3.9 (a), 

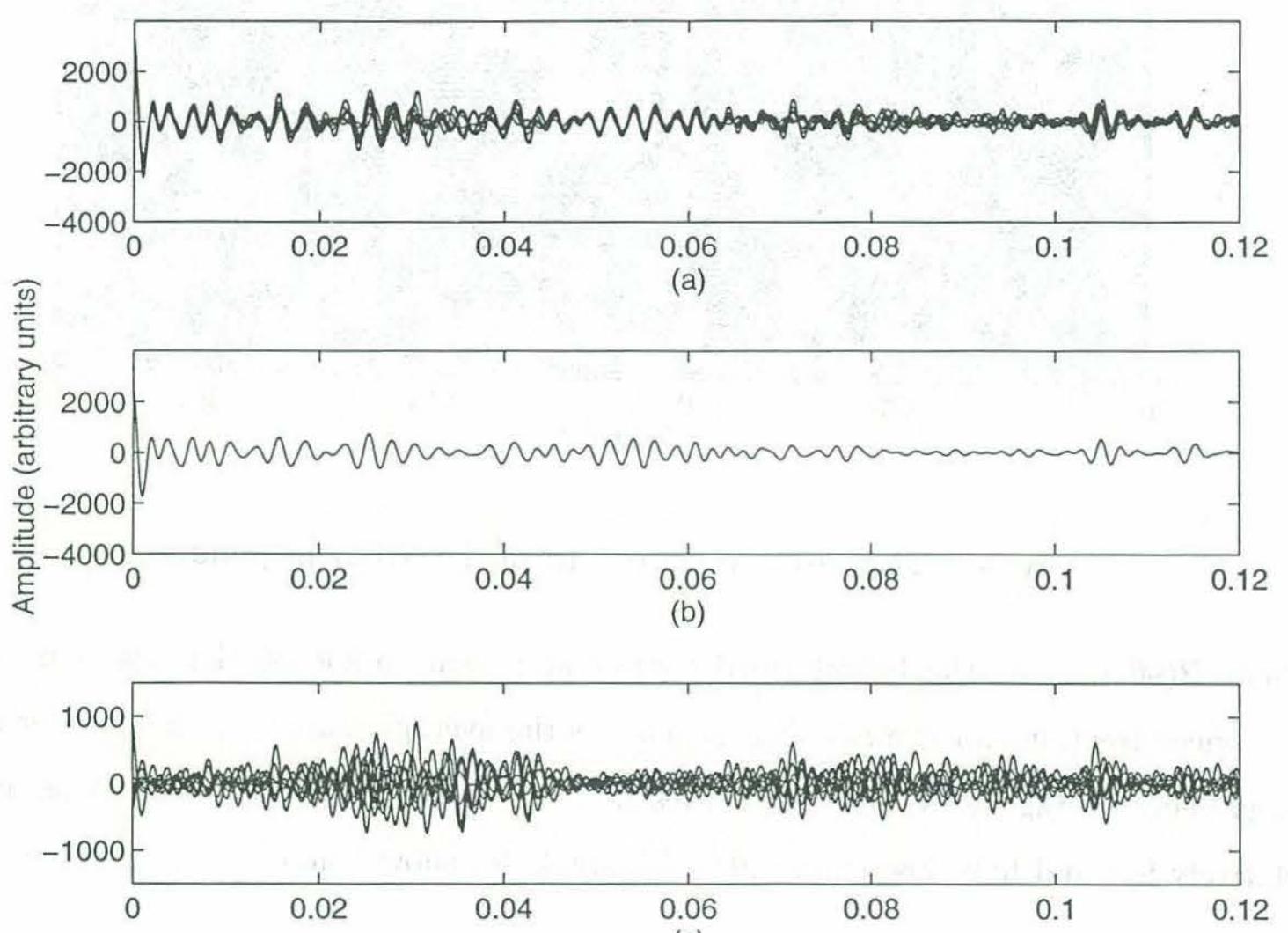

(c)

Time (sec)

Figure 3-8: (a) Beamformer outputs in the normal incidence direction for 8 pings at the east side of the sediment pond, (b) the mean of the signals in (a), (c) the signals in (a) with the mean removed. 

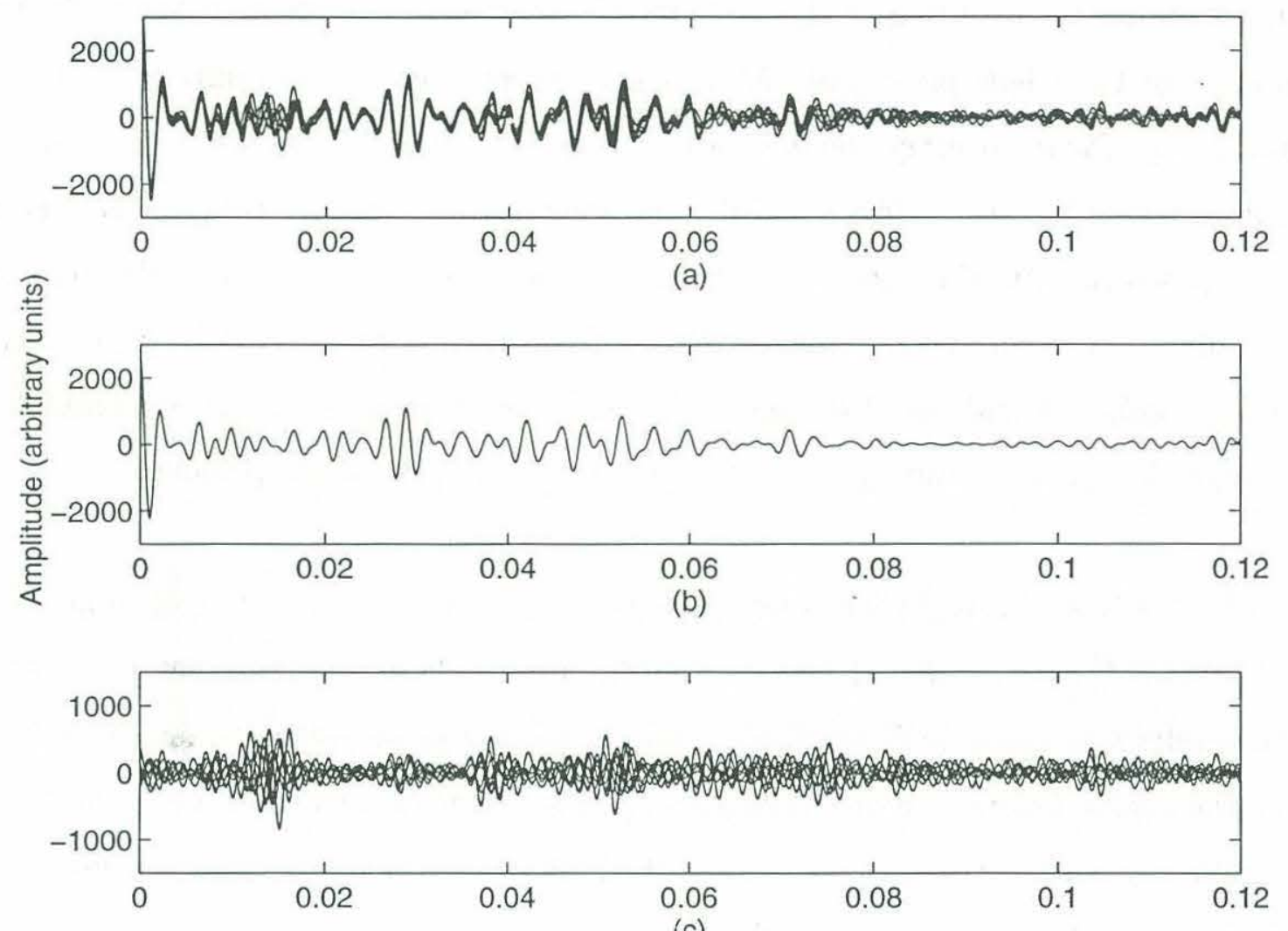

(c)

Time (sec)

Figure 3-9: (a) Beamformer outputs in the normal incidence direction for 8 pings at the west side of the sediment pond, (b) the mean of the signals in (a), (c) the signals in (a) with the mean removed. 
(b) and (c) show the normal incidence direction returns, the average signal and the difference signals of 8 pings, defined in the same way as in Figures 3.8 (a), (b) and (c). Notice that the poorly correlated part between $0.02 \mathrm{~s}$ and $0.045 \mathrm{~s}$, which shows up in Figure 3.8, disappears. However, the poorly correlated part between $0.065 \mathrm{~s}$ and $0.12 \mathrm{~s}$ still exists. Although there are small parts between $0.015 \mathrm{~s}$ and $0.018 \mathrm{~s}$, and between $0.04 \mathrm{~s}$ and $0.06 \mathrm{~s}$ showing some random features, we think that it is due to the gentle variation of the layer interfaces, which is evident in the sediment profiling of the whole pond [50]. Also, it can be seen that the amplitudes of these parts is high for the average signal shown in Figure 3.9(b), which is different from the random region between $0.065 \mathrm{~s}$ and $0.12 \mathrm{~s}$. Therefore, it appears that the sediment is composed mostly of horizontally stratified layers, except for two irregular regions lying about $18 \mathrm{~m}$ and $60 \mathrm{~m}$ beneath the sea floor, each approximatedly $20 \mathrm{~m}$ thick, with a nominal sound speed of $1530 \mathrm{~m} / \mathrm{s}$. The upper irregular region is absent for the west side of the sediment pond and the lower region is always present.

The two irregular regions can be seen more clearly if we employ an edge detection algorithm [51] to 9 groups of real data at the right side of the sediment pond [52]. The results are shown in figure 3.10. Although the lines in the plot may not represent the actual layers, the distributions of the horizontally stratified layers and the irregular regions are the same as we described above, which is additional evidence for the validity of the above-mentioned sediment structure hypothesis.

The conventional method of calculating bottom backscattering strength in a single source/single receiver geometry, as mentioned in Chap. 2, involves measuring the backscattering grazing angle with respect to the water-sediment interface, with the grazing angle obtained from:

$$
\theta=\sin ^{-1}\left(\frac{2 D}{c t}\right)
$$

where $D$ is the height of the source above the seafloor, $c$ is the sound speed in the water at the water-bottom interface, and $t$ is the time elapsed after source triggering. 


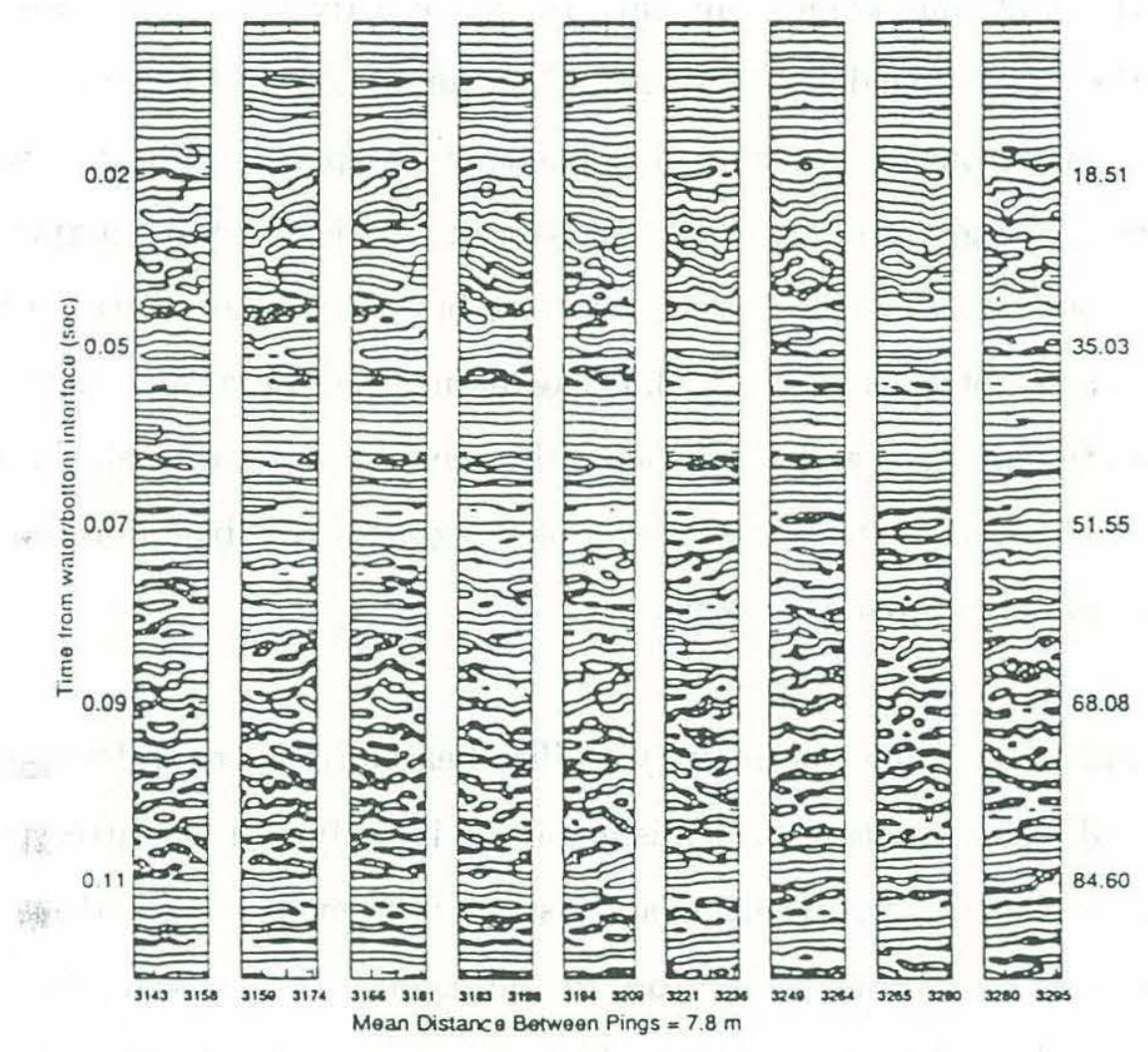

Figure 3-10: The inferred sediment structure using the edge detection algorithm for 9 groups of real data 
However, the above method is inaccurate for low-frequency backscattering, where penetration into the sediment is significant. The following are two cases where the conventional method fails: The first is when the sediment cover is thick and there are strong coherent reflections from internal horizontal layers. In this case, the backscattered signals at oblique angles can have the same arrival times as those reflection returns in the normal incidence direction. One cannot separate them by time gating. So it will be erroneous to associate the signals at one particular time $t$ with the returns at grazing angle $\theta$ by Eq. 3.3. The second case is when the major scatterers' locations are not at the water-bottom interface but at a certain distance beneath it, the grazing angle obtained from Eq. 3.3 is again incorrect because of the refraction of sound across the interface and $D$ not being the height of the source above the seafloor anymore. This demonstrates the necessity of using array and beamforming technique in the study of bottom backscattering.

The sediment profiling obtained by endfire beamforming provides a general picture of the sediment structure, which is helpful in identifying the scatterers' locations and understanding the scattering mechanisms. It is evident that these two irregular regions are the major contributors to the incoherent backscattering at normal and near-normal incidence directions. However, whether these irregular regions are responsible for scattering at oblique angles remains unknown at this point. The mechanisms for the scattering at normal and near-normal incidence directions and oblique directions may be different. We will concentrate on the scattering from oblique directions in the next section.

\subsection{Analysis of Signals in Oblique Directions}

We conclude from the last section that there are two irregular regions beneath the water-sediment interface, and otherwise the sediment is stratified with horizontally 
gently changing layers. Strong coherent reflections exist at the internal horizontal layer interfaces. Two irregular regions are found to dominate the incoherent backscattering in the normal and near-normal incidence directions.

In order to understand oblique bottom backscattering mechanisms, it is of great importance to identify the major scatterers' locations. With the help of bottom profiling using the endfire beamforming technique, we have obtained information about the sediment structure. The identified two irregular regions which dominate the random scattering in normal and near-normal incidence directions could also be the major sources for oblique backscattering. However, the two regions may have no significance on oblique backscattering. In this section, our goal is to determine the locations of the scatterers with principal contributions to the bottom scattering process and to estimate their scattering strengths.

The vertical line array in the experiment is used as the receiver, which offers the ability to discriminate signals in a desired direction from those in other directions. Combined with the temporal information, major scatterers within the sediment can be located. Because the bottom has a layered structure and the sediment thickness is up to $400 \mathrm{~m}$, strong coherent reflections from the layer interfaces in normal and nearnormal incidence directions may contaminate oblique backscattered signals through sidelobe leakage of the beamformer. This effect has to be alleviated in order to have a good estimate of oblique backscattering.

A method, which is a hybrid of multiple constraints beamforming and a subtraction procedure, will be introduced. The data recorded on each hydrophone is the sum of signals in all directions. By using endfire beamforming, one can get the signals from normal and near-normal incidence directions only. If this can be subtracted from the data at each hydrophone with careful adjustment of the time delay and amplitude, the difference signals left should be the returns in other than the normal 


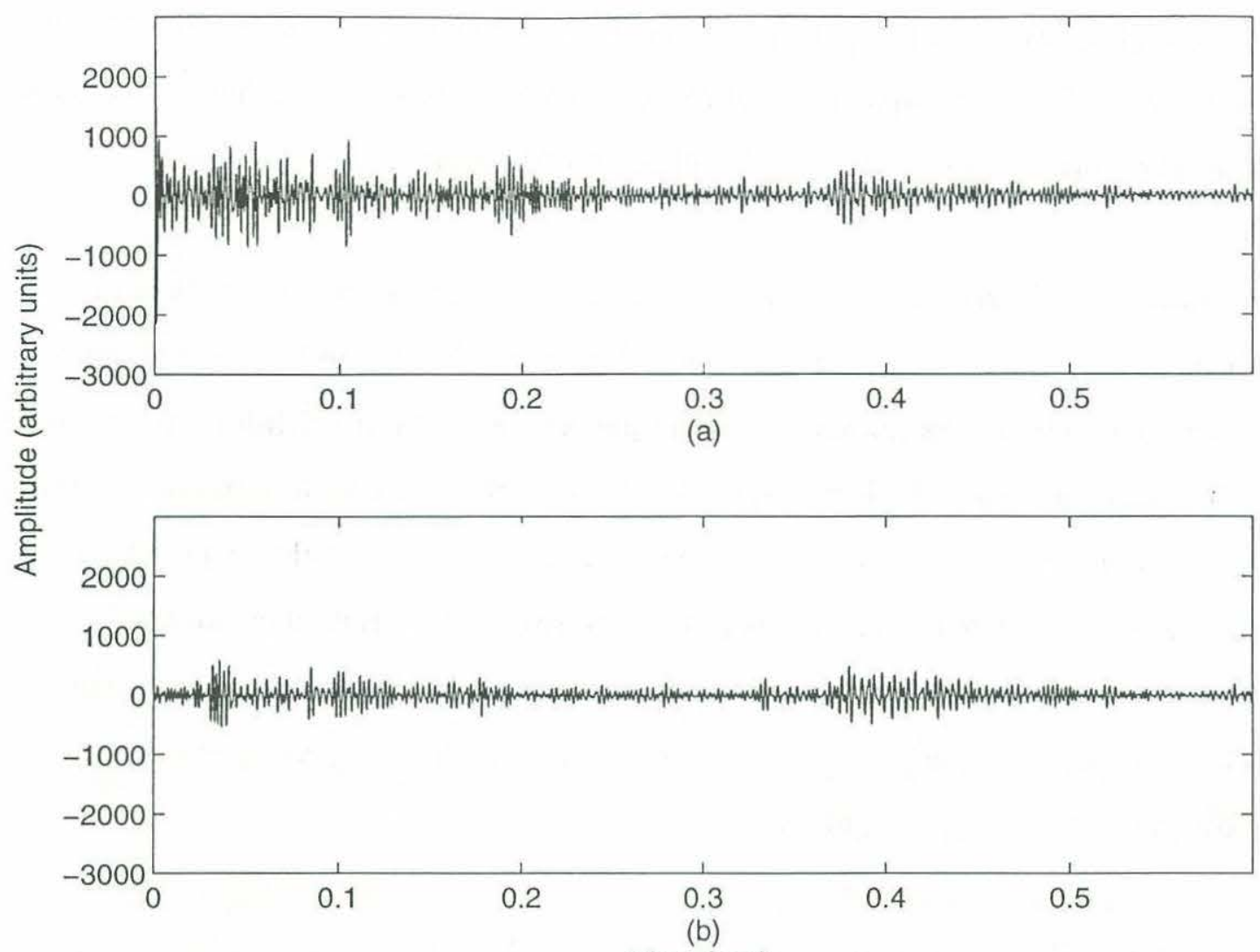

Time (sec)

Figure 3-11: For a typical ping: the time series at channel 1 (a) before and (b) after the subtraction.

and near-normal incidence directions. The formula to do this is:

$$
d_{i}(t)=p_{i}(t)-B(90, t, x), \quad i=1,2, \ldots, 24 .
$$

where $p_{i}(t)$ are the time series collected on each hydrophone. $B(90, t, x)$ is the endfire beamforming output. Therefore, the difference signal $d_{i}(t)$ is the scattered field at oblique angles only. Proper time delay and amplitude modification have been applied in the above subtraction process. Figure 3.11 (a) shows the time series at channel 1 for a typical ping before the subtraction and Figure 3.11 (b) shows the results after subtraction. Notice that the high amplitude peaks appearing before the rock bottom returns arrive at $0.4 \mathrm{~s}$ in Figure 3.11 (a) do not appear in Figure 3.11 (b), which indicates that the contamination from the normal and near-normal incidence directions has been greatly reduced. 
The next step is the acquisition of scattered signals in oblique directions. The multi-constraint beamforming technique is applied to the difference signals $d_{i}(t)$. As stated in Chapter 2, the multi-constraint beamformer has the advantage over the conventional one-constraint beamformer in rejecting normal and near-normal incidence returns. It can be used to obtain oblique backscattered signals with or without the above subtraction process in the presence of contamination from the normal and near-normal incidence directions. Nevertheless, it performs better when it is applied to the difference signals $d_{i}(t)$, as can be seen from the simulation results in Chapter 2 , although the computational load certainly will increase.

From figure 3.4, one can see that the endfire beamforming output is the signal from a 60 degree cone at $250 \mathrm{~Hz}$. As a result, the signals returned at grazing angles greater than 60 degrees will be affected by the subtraction process. Therefore, 60 degrees is chosen to be the upper limit for the look direction of the beamformer. Figure 3.12 shows the beamforming output for a typical ping collected at the east side of the sediment pond after Hilbert transforming [53] to get the envelope of the signal. The look direction is from 30 degrees to 60 degrees, with 5 degree increments. For the signals coming from grazing angles less than 25 degrees, peaks of backscattered signals are too low to distinguish from the background, so the beamforming output at those angles have not been included in this plot. It can be seen that there exist three groups of peaks for each look direction. The first two group center at approximately $0.04 \mathrm{~s}$ and $0.1 \mathrm{~s}$ on the 60 degree curve and move out gradually for smaller grazing angles. Assuming that these peaks occur due to horizontal interfaces and using the nominal sound speed of $1530 \mathrm{~m} / \mathrm{s}$ (ignoring the sound speed gradient at this point), we have the best fits indicated by the solid lines in figure 3.12 .

Again, we caution against making generalizations based on one ping. Therefore, we take the average of envelopes for the same 8 consecutive pings as those in Figure 3.8. A low-pass filter is used to smooth the curves in order to detect any potential 


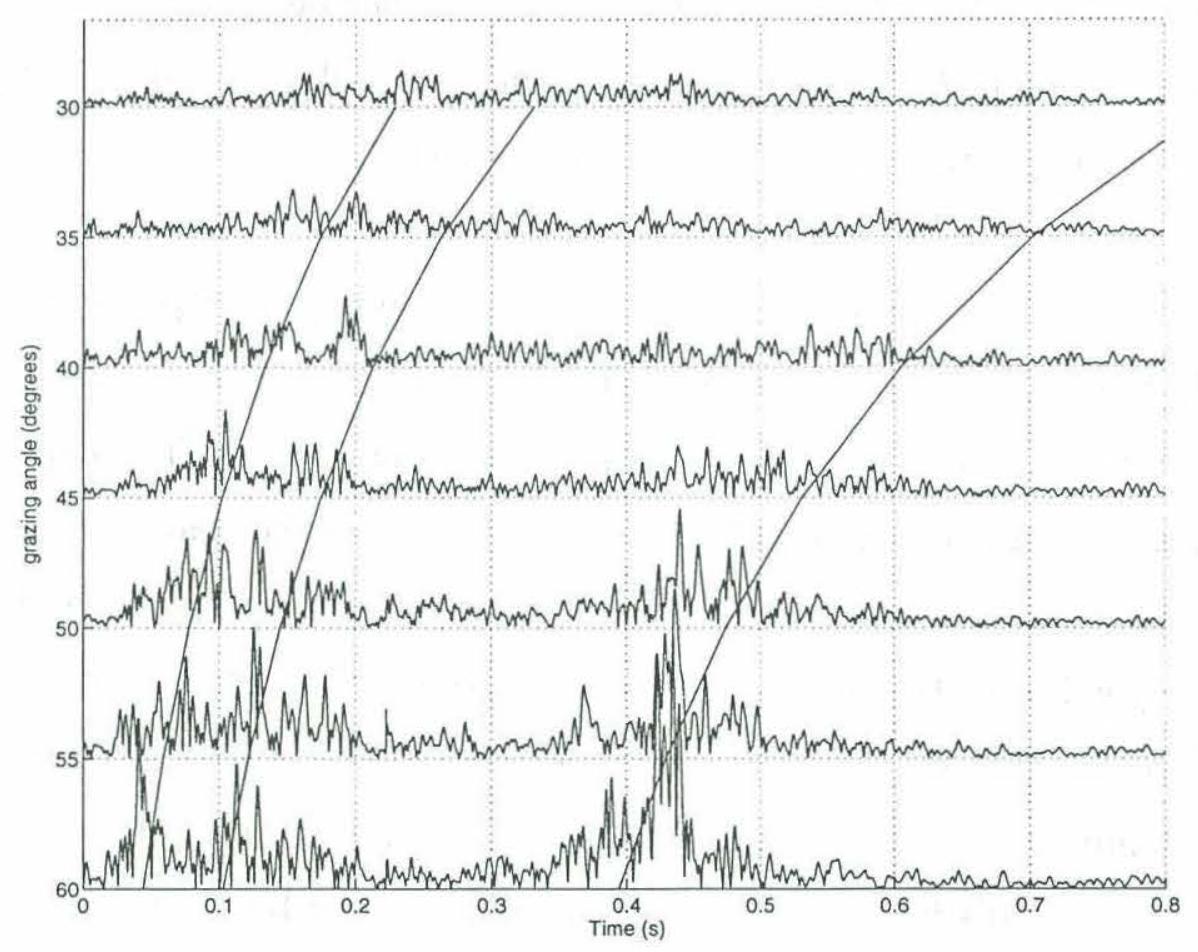

Figure 3-12: The envelope of oblique angle beamforming results for a single ping at the east side of the sediment pond (arbitrary units). 


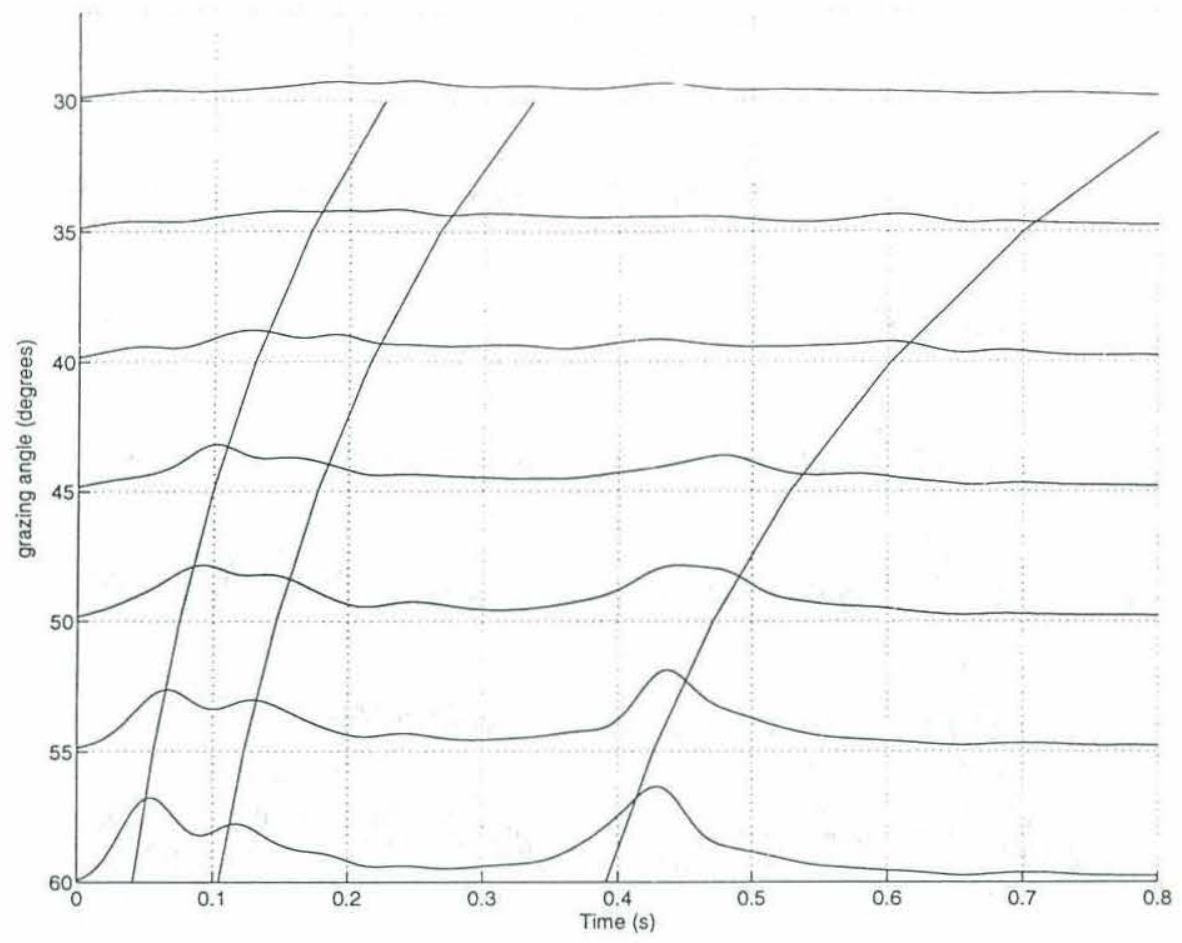

Figure 3-13: The average envelope of oblique angle beamforming results over 8 pings at the east side of the sediment pond (arbitrary units).

trend. As shown in Figure 3.13, it is clearer here that two solid lines fit the first two groups of peaks well. From the travel times, we find that these two arrivals correspond to the two irregular regions identified in the last section. The first solid line indicates that the interface is at a depth of $18 \mathrm{~m}$ and the second solid line indicates that the interface is at a depth of $60 \mathrm{~m}$, which can be seen in Figure 3.10 to be within the irregular regions. Therefore, we tentatively conclude that the backscattered field in oblique directions is also due to sediment inhomogeneities in the two irregular regions found in the last section.

Recall that for the data collected on the west side of the sediment pond as shown in Figure 3.9, only the lower irregular region is present. If the above hypothesis that inhomogeneities in the irregular region are the main influence on the oblique backscattered field is true, one will see only one group of peaks corresponding to the lower irregular region instead of two groups of peaks at the beginning part of the 


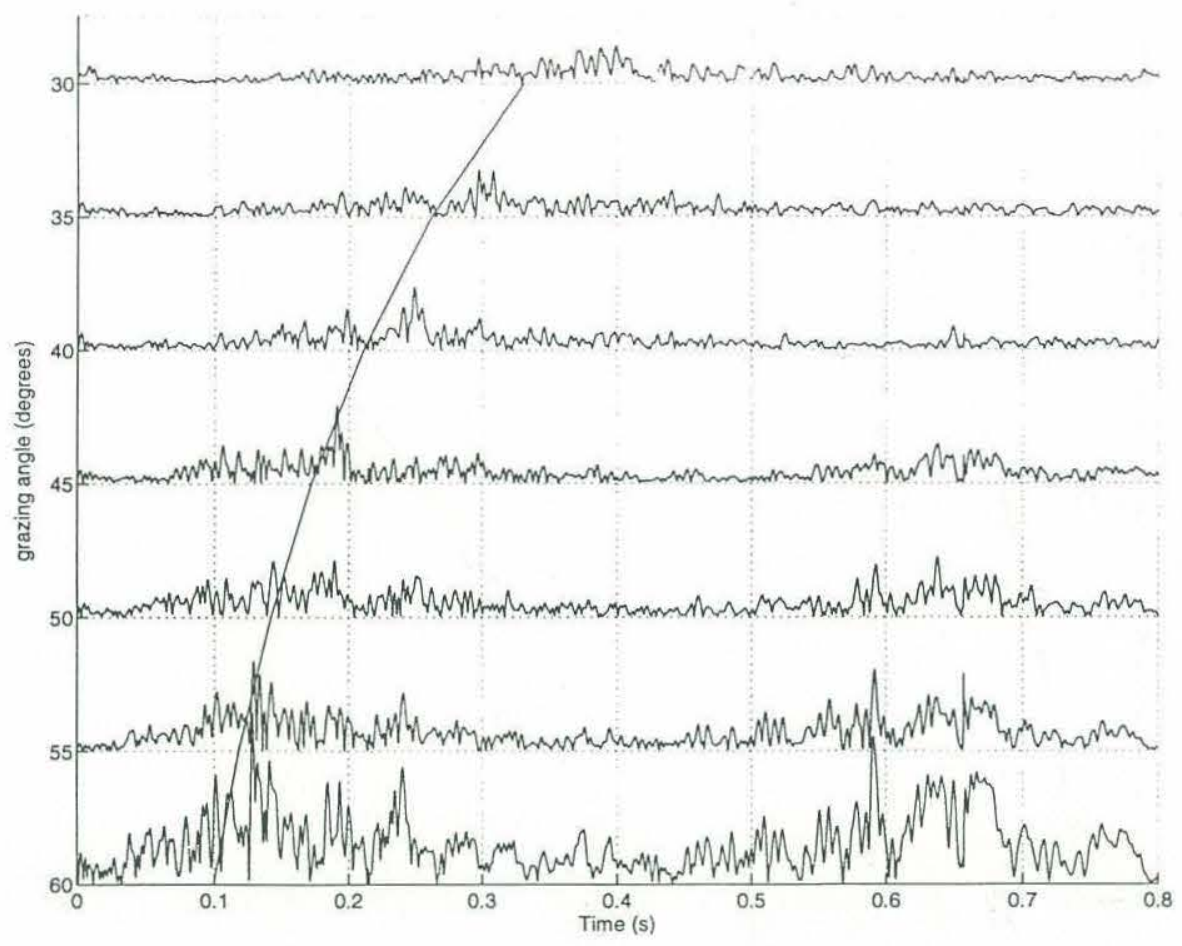

Figure 3-14: The envelope of oblique angle beamforming results for a single ping at the west side of the sediment pond (arbitrary units).

beamforming output. Figure 3.14 shows the envelope of beamformed results for a typical ping at the west side of the sediment pond. Figure 3.15 is the average of the envelopes for 8 consecutive pings, analogous to that in Figure 3.13. A smoothing procedure is also applied to the curves. The striking point here is that the first group of peaks does disappear, which means that the above hypothesis is correct. Therefore, it can be concluded that the irregular regions located in the last section are the dominant contributors to the incoherent backscattering in oblique directions.

The last group of peaks shown in Figure 3.12 is the scattering from the rough basement interface. If there were no sound speed gradient in the sediment, the peaks and the solid line would fit very well. However, we notice that the peaks vanish when the grazing angles are smaller than 40 degrees. This suggests that a sound speed gradient exists within the sediment. By assuming that the sound speed profile beneath the seafloor is linear with a constant gradient, the value of the gradient is estimated 


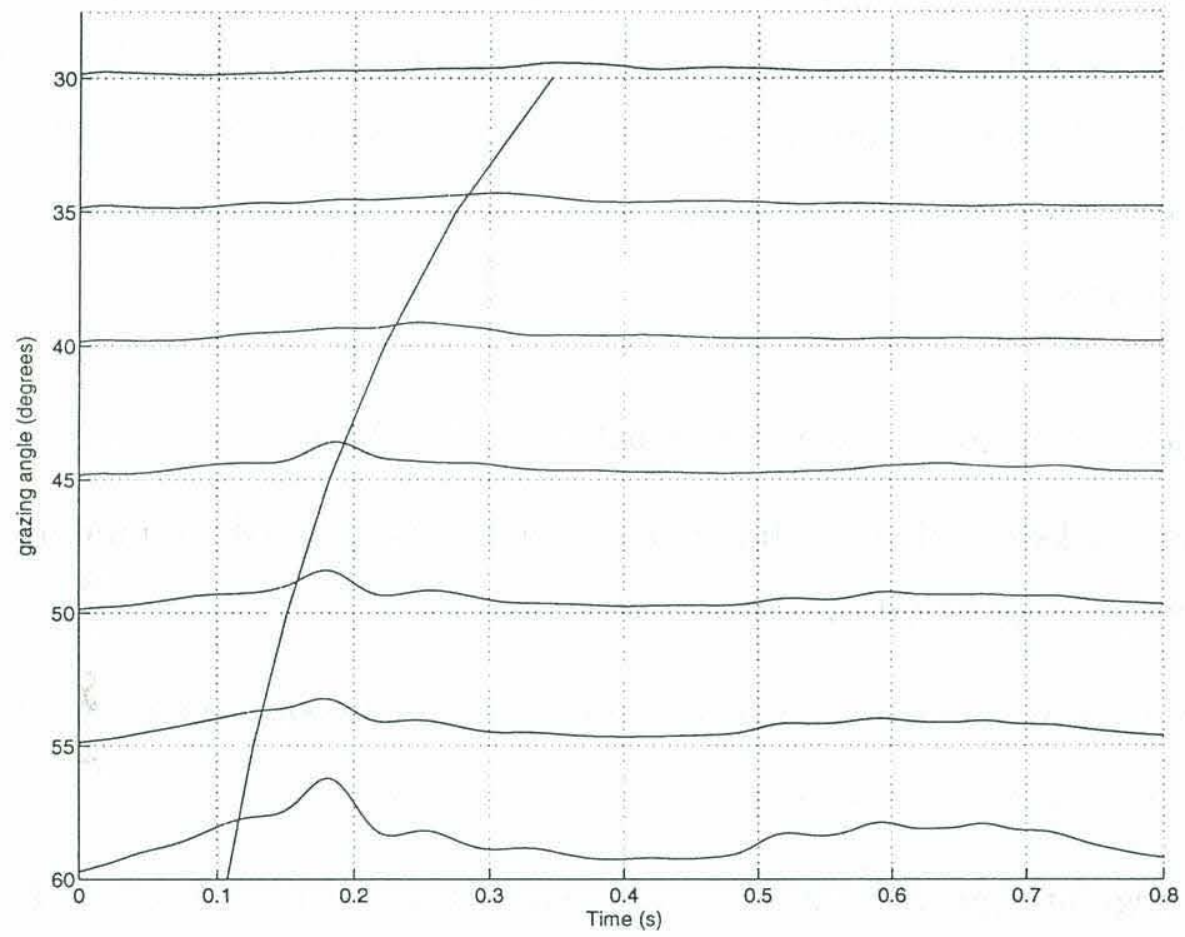

Figure 3-15: The average envelope of oblique angle beamforming results over 8 pings at the west side of the sediment pond (arbitrary units). 
to be 1.12 /s using a ray based method. As a result, all sound propagating at grazing angles smaller than 40 degrees will be refracted back toward the water-sediment interface before reaching the basement.

Finally, in our experiment, if the seafloor were rough enough to influence bottom backscattering, a peak should have shown up near $0 \mathrm{~s}$ in 60 degree grazing angle curve in Figure 3.12 and move out in the same way as other peaks. The absence of this peak suggests that the water-sediment interface is indeed flat and does not result in backscattering. Dominant bottom backscattering comes from beneath the seafloor. Also, the effect of internal laterally varying layer interfaces on bottom backscattering is insignificant. Otherwise, there should be more than two peaks corresponding to the layers' positions.

The major conclusions that we can reach from the above analysis are as follows:

- Bottom backscattering in oblique directions is dominated by inhomogeneities in irregular regions within the sediment.

- Backscattering due to volumetric inhomogeneities outside the irregular regions and interfaces roughness are relatively insignificant.

- The irregular regions within the sediment can be identified using the normal incidence returns because they are also the major contributors to scattering at normal and near normal incidence directions.

The scattering strength is an important measure in studies of scattering. Usually, the scattering strength is calculated as a function of grazing angle with respect to the water-sediment interface in the single source/single receiver geometry. The shortcomings of this measurement have been discussed previously. In our experiment, the scatterers' locations have been determined. Also, with the help of beamforming techniques, the backscattered signals can be extracted from each direction separately. We can therefore estimate the backscattering strength of each irregular layer. 
In our analysis, we will concentrate on the estimation of backscattering strengths for the two identified irregular regions. Since the two irregular regions are fairly thin, we can treat them as surface scatterers located at $18 \mathrm{~m}$ and $60 \mathrm{~m}$ beneath the seafloor. The backscattering strength, as commonly defined, which is shown in Chap. 2, is:

$$
B S S=10 \log _{10}\left[\frac{I^{s} r^{2}}{I^{i n} \cdot A}\right]
$$

where $I^{s}$ is the scattering intensity at the receiver, $I^{\text {in }}$ is the incident intensity at the scatterer, $r$ is the distance from the source to the scatterer, and $A$ is the insonified area. $I^{\text {in }}$ can be estimated from the direct arrival recorded on the first hydrophone. $I^{s}$ is angular dependent, which can be calculated from the beamformed output at each grazing angle. The average of many pings is taken and the curves are smoothed by a moving average. As a result, $I^{s}$ is calculated from the curves shown in figure 3.13 and figure 3.15. Since both the irregular regions are close to the seafloor, the geometric loss has no appreciable deviation from that obtained from spherical spreading, even though we predict a linear sound speed gradient in the sediment. Therefore, spherical spreading is used here in the backscattering strength calculation. For the same reason, sediment attenuation has not been taken into account in the calculation.

Figure 3.16 (a) and (b) show the backscattering strength as a function of grazing angle for the upper and lower scattering regions at the east side of the sediment pond, averaged over 8 pings (the same pings as those in Figure 3.8). The standard deviations of the backscattering strengths are also given as error bars. Figure 3.17 is similar to figure 3.16, except that it is for the lower scattering region at the west side of the sediment pond, averaged over 8 pings (the same pings as those in figure 3.9). The dashed lines in the figures are the best fit to Lambert's law [31]:

$$
B S S=10 \log _{10}\left(\mu \cdot \sin ^{2} \theta\right),
$$

where $\theta$ is the backscattering angle with respect to the scatterers' depth, and $\mu$ is the Mackenzie coefficient. In Figure 3.16, the Mackenzie coefficient is $-31 \mathrm{~dB}$ for the 


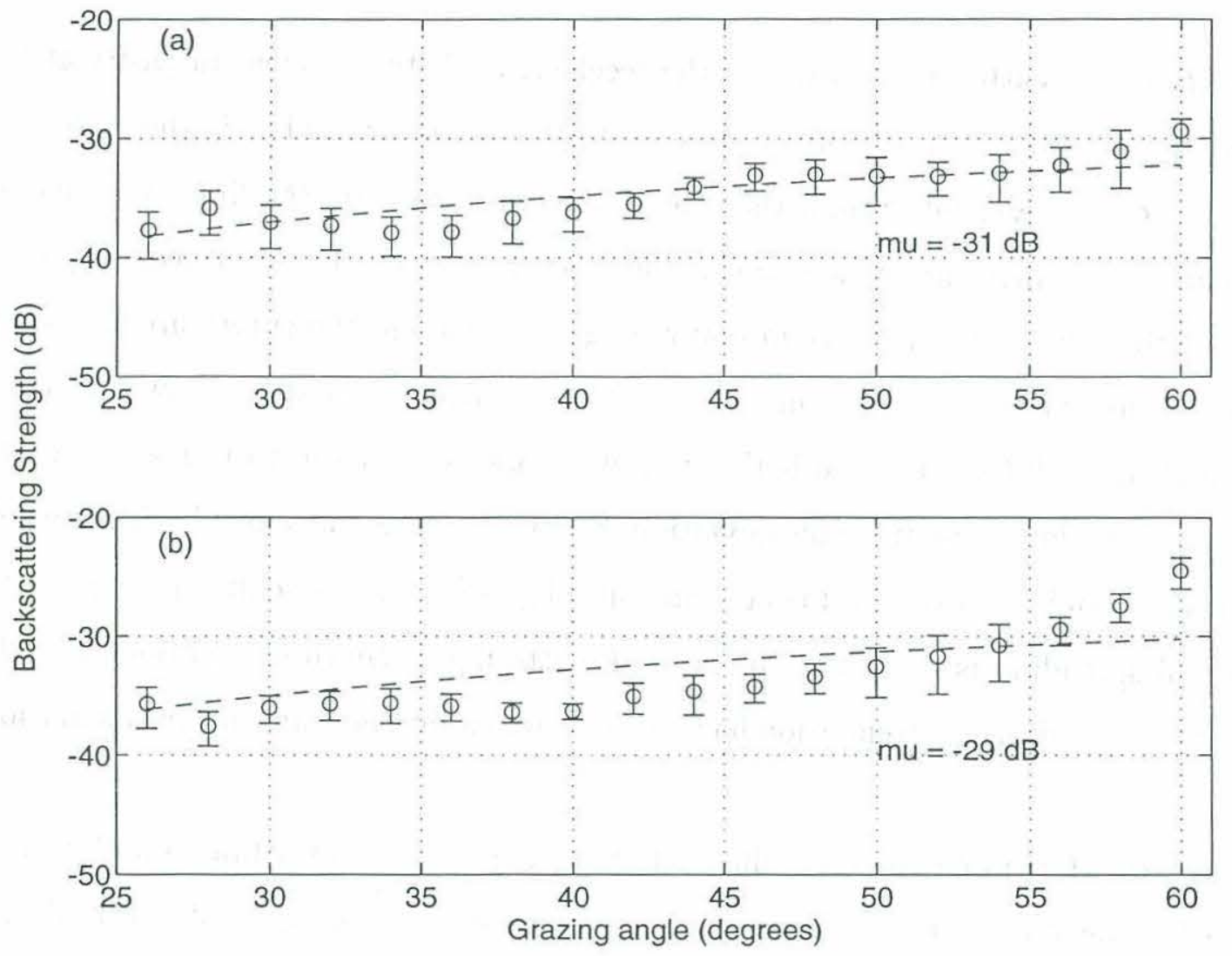

Figure 3-16: The backscattering strength with standard deviations (error bars) and the Lambert's law fit (dashed line) for the (a) upper and (b) lower irregular regions at the east side of the sediment pond. 


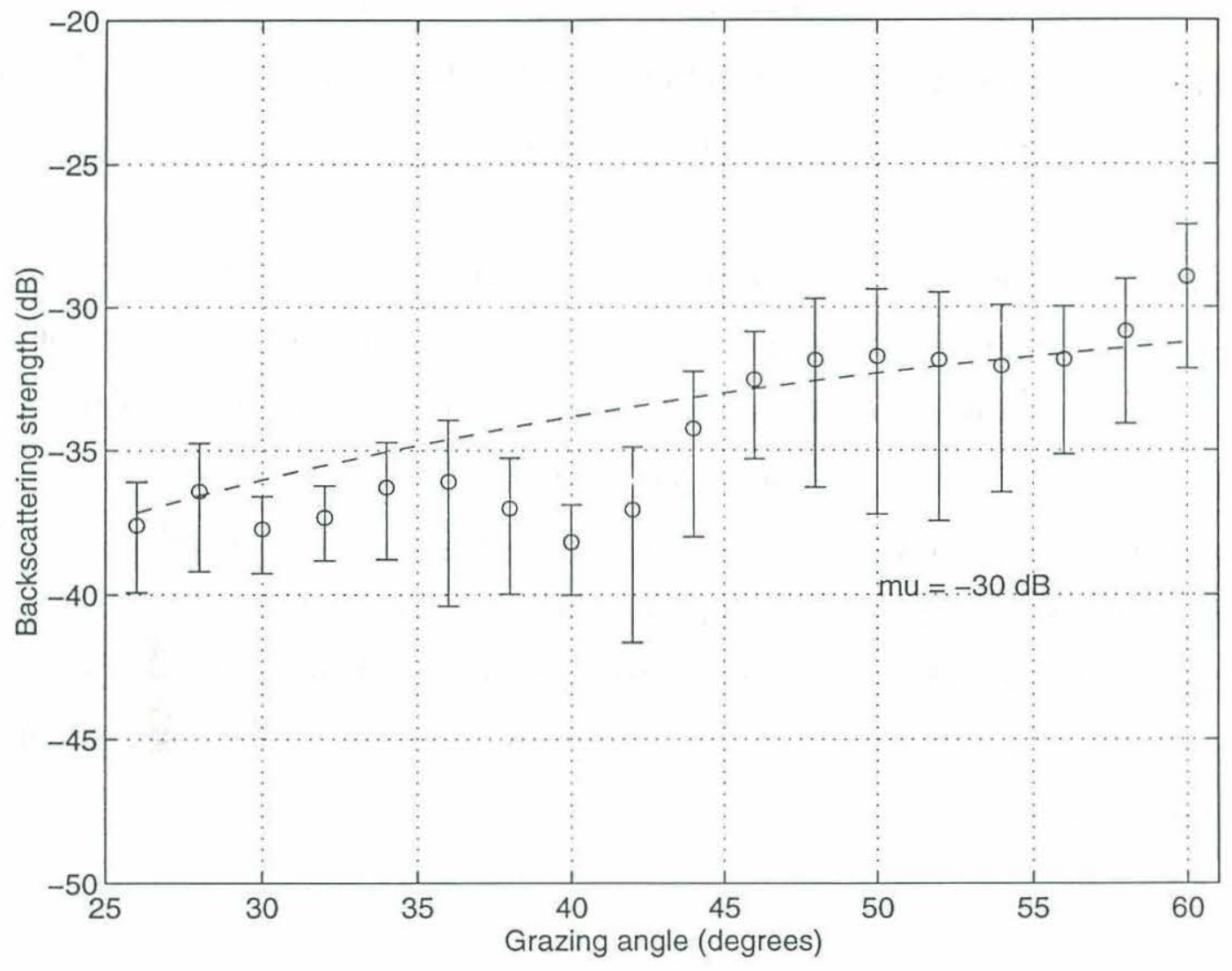

Figure 3-17: The backscattering strength with standard deviations (error bars) and the Lambert's law fit (dashed line) for the lower irregular region at the west side of the sediment pond. 
upper irregular region and $-29 \mathrm{~dB}$ for the lower one. In Figure 3.17, the Mackenzie coefficient is $-30 \mathrm{~dB}$ for the lower scattering region.

From the backscattering strengths that we have obtained above, it can be seen that the backscattering levels that result from inhomogeneities in the irregular regions within the sediment are comparable to those due to seafloor roughness only, where $\mu=-27 \mathrm{~dB}[31]$. It is of great importance to include the scattering from beneath the water-sediment interface in the evaluation of bottom backscattering, especially when the seafloor is relatively flat and the frequency is low.

In the modeling of bottom scattering, it is difficult to take into consideration the effect of the internal scattering regions, because knowledge of the locations, geological properties and distributions, and statistical characteristics for these irregular regions are very limited. The method that we have employed in the data analysis, i.e., using the normal incidence return to determine the locations of the irregular scattering regions, sheds some light on this aspect. However, since bottom scattering varies with bottom types and locations, more work needs to be done to test the generality of this method. It will be very helpful if more ground truth can be obtained for the scattering regions. 


\section{Chapter 4}

\section{Conclusions and Future Work}

\subsection{Conclusions}

Low frequency acoustic backscattering from the ocean bottom is different from high frequency backscattering. It is more complicated, because substantial penetration of acoustic energy into the bottom makes the subbottom inhomogeneities one possible significant contributor to bottom backscattering. It is very difficult to identify the characteristics of inhomogeneities that cause the reradiation of acoustic energy. Many experiments have been done, trying to reveal the bottom scattering mechanisms. However, the potential shortcomings of typical experimental configurations, (e.g. single source/single receiver) have limited the progress.

With a broadband, low-frequency source and an attached vertical receiving array deployed close to the seafloor, the recent ARSRP backscattering experiment conducted on the western flank of the Mid-Atlantic Ridge provided the opportunity to locate the dominant scatterers within the sediment, making valuable contributions to the understanding of the bottom backscattering process. The backscattering data analysis was the major task of this thesis.

Taking advantage of the vertical receiving array, a beamforming technique was employed to obtain the scattered signals from desired directions. Endfire beamforming 
was used to obtain the normal incidence returns from locations across the sediment pond. The sediment structure was determined by subbottom profiling. Then a hybrid of multiple constraints beamforming and a subtraction procedure was used to obtain the backscattered signals at oblique angles. Since strong reflections in the normal incidence direction due to layers in the sediment might have the same arrival time as the weak backscattered returns in oblique directions, they could not be separated simply by time gating. Even with conventional single constraint linear beamforming, the contamination of oblique scattering due to sidelobe leakage could be significant. Therefore, a subtraction procedure, which removed the normal incidence direction returns from the total received signals, was applied first, followed by a multiple constraints beamformer. The results were excellent.

From the revealed sediment structure, it could be seen that the sediment is stratified with gentle horizontal changes, except for two $20 \mathrm{~m}$ thick, irregular regions located about $18 \mathrm{~m}$ and $60 \mathrm{~m}$ beneath the seafloor. The striking point is that these two inhomogeneous regions were demonstrated to be the dominant contributors to the bottom backscattered field, both in the normal incidence and oblique directions. The backscattering strength as a function of grazing angle for each region was then estimated from the oblique angle beamforming output.

In conclusion, the internal inhomogeneous regions within the sediment were found to be the main influence on bottom backscattering. These irregular regions were successfully identified by sediment profiling using a vertical receiving array and a beamforming technique. However, more work remains to be done in order to achieve a better understanding of the scattering mechanisms and to reach the point of accurate prediction of bottom scattering. 


\subsection{Recommendations for Future Work}

Based on the results of analysing the ARSRP experimental data presented in this thesis, the following work is suggested for the future. The first is to develop a bottom backscattering model, taking into consideration the internal irregular layer effect. The knowledge of the dominant scatterers' locations should be included in the model, which is one step further compared with the traditional two half-space model. Major interest lies in the determination of the scattering mechanisms of the irregular layers, i.e., are they due to stacks of rough interfaces or a volumetric inhomogeneous distribution of density and compressibility variations?

Although two irregular regions have been identified containing dominant scatterers within the sediment, their geological properties remain unclear. It will be important to investigate the geological formation of these random regions, which will help to model bottom backscattering.

In the experimental data analysis, the vertical receiving array was steered in the normal incidence direction first to determine the sediment structure, and then steered in oblique directions to obtain the backscattering strength. The major scatterers could be located using this approach. It will be of great significance if this approach is generally valid for any seafloor with thick sediment cover. Hence, it is important to apply the same processing procedure to other datasets.

The basement beneath the sediment has a rough interface. The backscattering from the basement interface, which is another major contributor to the total bottom scattering, remains to be investigated.

Some of the geoacoustic parameters such as sound velocity gradient and attenuation in the sediment were not rigorously considered due to the lack of ground truth data. It will improve the accuracy of the processing if they could be estimated and 
incorporated in the future analysis. 


\section{Bibliography}

[1] D. R. Jackson, D. P. Winebrenner, and A. Ishimaru. Application of the composite roughness model to high-frequency bottom backscattering. J. Acoust. Soc. Am., 79:1410-1422, 1986.

[2] J. A. Ogilvy. Theory of Wave Scattering from Random Rough Surfaces. Adam Hilger, Bristol, 1991.

[3] J. H. Stockhausen. Scattering from the volume of an inhomogeneous half-space. Naval Research Establishment report,63/9, 1963. Canada.

[4] P. M. Morse. Vibration and Sound. McGraw-Hill, New York, 1948.

[5] A. W. Nolle, W. A. Hoyerand, J. F. Mifsud, W. R. Runyan, and M. B. Ward. Acoustic properties of water-filled sands. J. Acoust. Soc. Am., 35:1394-1408, 1963.

[6] P. A. Crowther. Some statistics of the sea-bed and acoustic scattering therefrom. In N. G. Pace, editor, Acoustics and the Sea-Bed, pages 147-155. Bath Univ., Bath,England, 1983.

[7] E. Y. Kuo. Wave scattering and transmission at irregular surfaces. J. Acoust. Soc. Am., 36:2135-2142, 1964.

[8] Yu. Yu. Zhitkovskii. Comparison of the sharp-pulse (explosion) and CW-burst methods for measuring the intensity of sound scattering by the ocean bottom. In Eighth All-Union Acoustics Conf., page 88, 1973. in Russian. 
[9] V. I. Volovov and Yu. Yu. Zhitkovskii. Reflection and scattering of sound by the ocean bottom. In L. M. Brekhovskikh, editor, Ocean Acoustics, page 395. Nauka, Moscow, 1974. Part 6, in Russian.

[10] A. N. Ivakin and Yu. P. Lysanov. Theory of underwater sound scattering by random inhomogeneities of the bottom. Sov. Phys. Acoust., 27:61-64, 1981.

[11] Yu. P. Lysanov. Geoacoustic model of the upper sedimentary layer in shallow seas. Dokl. Adad. Nauk SSSR, 251:714, 1980.

[12] A. N. Ivakin and Yu. P. Lysanov. Underwater sound scattering by volume inhomogeneities of a bottom bounded by a rough surface. Sov. Phys. Acoust., $27: 212-215,1981$.

[13] A. N. Ivakin. Sound scattering by random inhomogeneities of stratified ocean sediments. Sov. Phys. Acoust., 32:492-496, 1986.

[14] P. D. Mourad and D. R. Jackson. High frequency sonar equation models for bottom backscatter and forward loss. In Oceans '89 Proceedings, IEEE, pages 1168-1175, New York, 1989.

[15] E. L. Hamilton. Compressional wave attenuation in marine sediments. Geophysics, 37:620-646, 1972.

[16] P. D. Mourad and D. R. Jackson. A model/data comparison for low-frequency bottom backscatter. J. Acoust. Soc. Am., 94:344-358, 1993.

[17] P. C. Hines. Theoretical model of acoustic backscatter from a smooth seabed. J. Acoust. Soc. Am., 88:324-334, 1990.

[18] L. A. Chernov. Wave Propagation in a Random Medium. McGraw-Hill, New York, 1975. Translated from Russian by R. A. Silverman.

[19] D. Tang. Acoustic Wave Scattering from a Random Ocean Bottom. PhD thesis, MIT/WHOI Joint Program, 1991. 
[20] D. Tang and G. V. Frisk. Plane-wave reflection from a random fluid half-space. J. Acoust. Soc. Am., 90:2751-2756, 1991.

[21] D. Tang and G. V. Frisk. Spectral parameterization of sound scattering from a random ocean bottom. J. Acoust. Soc. Am., 92:2792-2799, 1992.

[22] D. Tang and G. V. Frisk. Spatial correlation of acoustic wave scattered from a random ocean bottom. J. Acoust. Soc. Am., 97:2783-2803, 1995.

[23] A. P. Lyons, A. L. Anderson, and F. S. Dwan. Acoustic scattering from the seafloor: Modeling and data comparison. J. Acoust. Soc. Am., 95:2441-2451, 1994.

[24] W. A. Kuperman and Henrik Schmidt. Self-consistent perturbation approach to rough surface scattering in stratified elastic media. J. Acoust. Soc. Am., 86:15111522, October 1989.

[25] J.-Y. Liu, H. Schmidt, and W. A. Kuperman. Effect of a rough seabed on the spectral composition of deep ocean infrasonic ambient noise. J. Acoust. Soc. Am., 93:753-769, 1993.

[26] K. D. LePage. Elastic scattering in oceanic waveguides. $\mathrm{PhD}$ thesis, Massachusetts Institute of Technology, 1993.

[27] Henrik Schmidt and W. A. Kuperman. Spectral respresentations of rough inteface reverberation in stratified ocean waveguides. J. Acoust. Soc. Am., 97:21992209, April 1995.

[28] Raymond Lim. Multiple scattering by many bounded obstacles in a multilayered acoustic medium. J. Acoust. Soc. Am., 92:1593-1612, October 1991.

[29] R. H. Hackman and G. S. Sammelemann. Acoustic scattering in an inhomogeneous waveguide: Theory. J. Acoust. Soc. Am., 80:1447-1458, November 1986.

[30] P. Gerstoft and H. Schmidt. A boundary element approach to ocean seismoacoustic facet reverberation. J. Acoust. Soc. Am., 89:1629-1642, April 1991. 
[31] K. V. Mackenzie. Bottom reverberation for 530- and 1030-cps sound in deep water. J. Acoust. Soc. Am., 33:1498-1504, 1961.

[32] H. M. Merklinger. Bottom reverberation measured with explosive charges fired deep in the ocean. J. Acoust. Soc. Am., 44:508-513, 1968.

[33] A. V. Bunchuk and Yu. Yu. Zhitkovskii. Sound scattering by the ocean bottom in shallow-water regions (review). Sov. Phys. Acoust., 26:363-370, 1980.

[34] H. Boehme, N. P. Chotiros, L. D. Rolleigh, S. P. Pitt, A. L. Garcia, T. G. Goldsberry, and R. A. Lamb. Acoustic backscattering at low grazing angles from the ocean bottom. part i. bottom backscattering strength. J. Acoust. Soc. Am., 77:962-974, 1984.

[35] J. R. Preston and T. Akal. Analysis of backscattering data in the Tyrrhenian sea. J. Acoust. Soc. Am., 87:119-134, 1989.

[36] P. C. Hines and P. J. Barry. Measurements of acoustic backscatter from the Sohm Abyssal Plain. J. Acoust. Soc. Am., 92:315-323, 1992.

[37] D. Tang, W. C. Burgess, S. D. Rajan, C. J. Sellers, G. V. Frisk, and D. Li. A summary of the direct-path scattering experiments in the ARSRP sediment pond and some preliminary results. In Fall ARSRP Research Symposium, December 1993.

[38] S. Fu. Master's thesis, University of Hawaii, 1994.

[39] W. K. Stewart, Private Communication.

[40] J. F. Gettrust, J. H. Ross, and M. M. Rowe. Development of a low frequency, deep tow geoacoustics system. Sea Technology, September 1991.

[41] B. D. Van Veen and K. M. Buckley. Beamforming: a versatile approach to spatial filtering. IEEE ASSP Magzine, April 1988. 
[42] M. E. Weber and R. Heisler. A frequency-domain beamforming algorithm for wideband, coherent signal processing. J. Acoust. Soc. Am., 76:1132-1144, October 1984 .

[43] A. B. Baggeroer, Lecture Notes, MIT.

[44] H. Schmidt, A. B. Baggeroer, W. A. Kuperman, and E. K. Sheer. Environmentally tolerant beamforming for high-resolution matched field processing: Deterministic mismatch. J. Acoust. Soc. Am., 88:1851-1862, 1990.

[45] B. E. Howard and J. M. Syck. Calculation of the shape of a towed underwater acoustic array. IEEE J. Oceanic Eng., 17:193-203, April 1992.

[46] N. C. Wyeth. Methods of array element localization for a towed underwater acoustic array. IEEE J. Oceanic Eng., 19:128-133, January 1994.

[47] R. J. Urick. Principles of Underwater Sound. McGraw-Hill, New York, 1983.

[48] Marc Gensane. A statistical study of acoustic signals backscattered from the sea bottom. IEEE J. Oceanic Eng., 14:84-93, January 1989.

[49] T. G. Muir, R. J. Wyber, J. B. Lindberg, and L. A. Thompson. Seismic profiling with a parametric, self-demodulated Ricker wavelet. In H. M. Merklinger, editor, Progress in Underwater Acoustics, pages 189-206. Plenum Press, New York and London, 1986.

[50] J. A. Showalter, Private Communication.

[51] J. S. Lim. Two-Dimensional Signal and Image Processing. Prentice-Hall, Englewood Cliffs, N.J., 1990.

[52] C. J. Sellers, Private Communication.

[53] A. V. Oppenheim and R. W. Schafer. Discrete-Time Signal Processing. PrenticeHall, Englewood Cliffs, N.J., 1989. 